\title{
THE ELLIPTIC INTEGRAL IN ELECTROMAGNETIC THEORY*
}

BY

\section{ALFRED GEORGE GREENHILL}

The analytical complexity in the reduction of the elliptic integral in electromagnetism, as well as in most dynamical problems, arises in consequence of the appropriate integral of the third kind being of the circular form in LEGENDRE's terminology, and the elliptic parameter of $\mathrm{J}_{\mathrm{ACOBI}}$ is then a fraction of the imaginary period.

The expression of the integral by the theta function would imply then a complex argument, and a table of the theta function would not be of complete utility unless made a triple entry table.

When required in a problem of electromagnetism it is the complete third elliptic integral which usually is sufficient for a solution, and this, as shown by LEGENDRE, can be expressed by elliptic integrals of the first and second kind, complete and incomplete; and for these the Table IX of LEGENDre provides the material for a numerical evaluation.

As an important application we may cite the calculation of the mutual induction of two coaxial helices, employed in the ampère balance designed for weighing their electromagnetic attraction by the late Viriamu Jones and Professor ArrTon, and so arriving at an independent determination of the electrical units.

The present investigation was undertaken in the lifetime of Professor Viriamu JONES, with the object of exhibiting the result of his complete third elliptic integral in its simplest form, suitable for immediate numerical computation, and also to reconcile the conflicting notation of different writers on the subject by adopting Maxwell's Electricity and Magnetism as the standard.

Incidentally the quadric transformation of LANDEN is required here so frequently that a digression has been made on the theory, and an elucidation submitted of its essential geometrical interpretation.

The references in the course of the work will be chiefly to -

MAXWELL, Electricity and Magnetism.

WeBster, Electricity and Magnetism.

Gray, Absolute Measurements in Electricity and Magnetism.

CaYlex, Proceedings of the London Mathematical Society, vol.6.

* Presented to the Society September 5, 1907. Received for publication January 29, 1907. 
Viriamu Jones, Philosophical Magazine (1889), Philosophical Transactions (1891), Proceedings of the Royal Society (1897).

Mrnchin, Philosophical Magazine (1893), (1894).

Burnside, Messenger of Mathematics, vol. 20 (1891).

Coleridge Farr, Proceedings of the Royal Society (1898).

NagaOKa, Journal of the Tokyo College of Science, 16 (1903).

Coffrn, Rosa, and CoHen, Electromagnetic Integrals, Bulletin of the Bureau of Standards (1906).

Alexander Russell, Magnetic Field of the Helix, Philosophical Magazine, April, 1907.

\section{Notation and preliminary theory.}

1. The elliptic integrals of the I, II, and III kind (abbreviated in the sequel to I. E. I. ; II. E. I. ; III. E. I.) are composed of differential elements of the form

$$
\begin{aligned}
& \frac{d s}{\sqrt{ } S}, \quad(s-\sigma) \frac{d s}{\sqrt{ } S}, \quad \frac{1}{s-\sigma} \frac{d s}{\sqrt{ } S}, \\
& S=4 \cdot s-s_{1} \cdot s-s_{2} \cdot s-s_{3}, \quad s_{1}>s_{2}>s_{3} ;
\end{aligned}
$$

but for analytical simplification it is convenient to normalise them to zero degree by an appropriate factor, so that the elements may be written

$$
\begin{gathered}
\frac{\sqrt{ }\left(s_{1}-s_{3}\right)}{\sqrt{ } S} \frac{d s}{}, \quad \frac{s-\sigma}{\sqrt{ }\left(s_{1}-s_{3}\right)} \frac{d s}{\sqrt{ } S}, \quad \frac{\frac{1}{2} \sqrt{ } \Sigma}{s-\sigma} \frac{d s}{\sqrt{ } S}, \\
\Sigma=4 \cdot \sigma-s_{1} \cdot \sigma-s_{2} \cdot \sigma-s_{3} ;
\end{gathered}
$$

and in the circular form of the III. E. I. the expression $\Sigma$ is negative and the normalising factor is changed to $\frac{1}{2} V(-\Sigma)$.

The same normalising is required with the elementary circular or hyperbolic integral which arises when $S$ is of the second or first degree, in consequence of $s_{1}$, or $s_{1}$ and $s_{2}$ being made infinite, so that we take

$$
S=4 \cdot s-s_{2} \cdot s-s_{3}, \quad \text { or } \quad 4 \cdot s-s_{3} ;
$$

and the integral corresponding to the III. E. I. becomes

$$
\begin{aligned}
& \int \frac{V\left(s_{2}-\sigma \cdot \sigma-s_{3}\right)}{s-\sigma} \frac{d s}{\sqrt{ }\left(4 \cdot s-s_{2} \cdot s-s_{3}\right)}=\tan ^{-1} \sqrt{ }\left(\frac{s_{2}-\sigma}{\sigma-s_{3}} \cdot \frac{s-s_{3}}{s-s_{2}}\right), \\
& \int \frac{V\left(s_{3}-\sigma\right)}{s-\sigma} \frac{d s}{V\left(4 \cdot s-s_{3}\right)}=\sin ^{-1} \sqrt{\frac{s-s_{3}}{s-\sigma}}=\tan ^{-1} \sqrt{\frac{s-s_{3}}{s_{3}-\sigma}}
\end{aligned}
$$

with corresponding hyperbolic-logarithmic forms. 
The standard III. E. I. adopted in the sequel is written

$$
\int \frac{\frac{1}{2} v(-\Sigma)}{s-\sigma} \frac{d s}{\sqrt{ } S}
$$

in which the sign of $\Sigma$ is to be changed in the infrequent case which arises when the hyperbolic-logarithmic III. E. I. is to be employed; and it is preferable to keep to this form of the integral of an algebraic function, and to settle the sequence of magnitude of

$$
\sigma, s, s_{1}, s_{2}, s_{3},
$$

before proceeding to a reduction in the notation of WeIERSTRASs, JACOBI, and LEGENDRE to their standard form, if requisite for comparison.

In an electromagnetic application it is the complete III. E. I. which gives the solution, and then the limits of integration are $\infty, s_{1}, s_{2}, s_{3},-\infty$; and now the great theorem discovered by LEGENDRE (Fonctions elliptiques, I, chap. 23) enables us to express the result by incomplete integrals of the $I$ and II kind, the I. E. I. and II. E. I., on which a digression is made at the outset.

2. The jacobian quarter periods, $K$ and $K^{\prime}$, are defined by the complete integrals

$$
K=\int_{s_{1}, s_{3}}^{\infty, s_{2}} \frac{V\left(s_{1}-s_{3}\right) d s}{V S}, \quad K^{\prime}=\int_{s_{2},-\infty}^{s_{1}, s_{8}} \frac{V^{\prime}\left(s_{1}-s_{3}\right) d \sigma}{V(-\Sigma)} ;
$$

and the incomplete integrals may be written

$$
h K=\int_{s, s_{3}}^{\infty, s} \frac{V\left(s_{1}-s_{3}\right) d s}{V S}, \quad f K^{\prime}=\int_{\sigma,-\infty}^{s_{1}, \sigma} \frac{V\left(s_{1}-s_{3}\right) d \sigma}{V(-\Sigma)},
$$

where $h$ and $f$ are real proper fractions defined by the ratios

$$
h=\int_{s, s_{3}}^{\infty, s} \div \int_{s_{1}, s_{3}}^{\infty, s_{2}} \frac{d s}{\sqrt{ } S}, \quad f=\int_{\sigma,-\infty}^{s_{1}, \sigma} \div \int_{s_{2},-\infty}^{s_{1}, s_{3}} \frac{d \sigma}{V(-\Sigma)} .
$$

In the jacobian notation

and when

$$
k^{2}=\frac{s_{2}-s_{3}}{s_{1}-s_{3}}, \quad k^{\prime 2}=\frac{s_{1}-s_{2}}{s_{1}-s_{3}},
$$

$$
\infty>s>s_{1}, \quad \text { or } \quad s_{2}>s>s_{3} \text {, }
$$

$$
\mathrm{sn}^{2} h K=\frac{s_{1}-s_{3}}{s-s_{3}}, \quad \text { or } \quad \frac{s-s_{3}}{s_{2}-s_{3}},
$$

$$
\operatorname{cn}^{2} h K=\frac{s-s_{1}}{s-s_{3}}, \quad \text { or } \quad \frac{s_{2}-s}{s_{2}-s_{3}},
$$


while with

$$
\operatorname{dn}^{2} h K=\frac{s-s_{2}}{s-s_{3}}, \quad \text { or } \quad \frac{s_{1}-s}{s_{1}-s_{3}} ;
$$

$$
s_{1}>\sigma>s_{2}, \quad \text { or } \quad s_{3}>\sigma>-\infty \text {, }
$$

$$
\operatorname{sn}^{2} f K^{\prime}=\frac{s_{1}-\sigma}{s_{1}-s_{2}}, \quad \text { or } \quad \frac{s_{1}-s_{3}}{s_{1}-\sigma},
$$

$$
\operatorname{cn}^{2} f K^{\prime}=\frac{\sigma-s_{2}}{s_{1}-s_{2}}, \quad \text { or } \quad \frac{s_{3}-\sigma}{s_{1}-\sigma}
$$$$
\operatorname{dn}^{2} f K^{\prime}=\frac{\sigma-s_{3}}{s_{1}-s_{3}}, \quad \text { or } \quad \frac{s_{2}-\sigma}{s_{1}-\sigma} \text {. }
$$

It is the advantage of the use of the fraction $h$ or $f$ of the period $K$ or $K$ that the corresponding modulus $k$ or $k^{\prime}$ is indicated thereby and need not be written down.

In the notation given already in equations (2) the incomplete I. E. I. can be written

$$
\begin{aligned}
h K=\int_{s_{1}, s_{3}}^{\infty, s} \frac{V\left(s_{1}-s_{3}\right) d s}{\sqrt{ }}=\mathrm{sn}^{-1} \sqrt{\frac{s_{1}-s_{3}}{s-s_{3}},} & \mathrm{sn}^{-1} \sqrt{\frac{s-s_{3}}{s_{2}-s_{3}}} \\
(1-h) K=\int_{s_{1}, s}^{s, s_{2}} \frac{V\left(s_{1}-s_{3}\right) d s}{\sqrt{ } S}=\mathrm{sn}^{-1} \sqrt{\frac{s-s_{1}}{s-s_{2}},} & \mathrm{dn}^{-1} \sqrt{\frac{s_{1}-s_{2}}{s_{1}-s}}
\end{aligned}
$$

and for the complementary modulus $k^{\prime}$,

$$
f K^{\prime}=\int_{\sigma,-\infty}^{s_{1}, \sigma} \frac{V\left(s_{1}-s_{3}\right) d \sigma}{V(-\Sigma)}=\mathrm{sn}^{-1} \sqrt{\frac{s_{1}-\sigma}{s_{1}-s_{2}}}, \quad \mathrm{sn}^{-1} \sqrt{\frac{s_{1}-s_{3}}{s_{1}-\sigma}} ;
$$

$$
(1-f) K^{\prime}=\int_{s_{2}, \sigma}^{\sigma, s_{3}} \frac{V\left(s_{1}-s_{3}\right) d \sigma}{\sqrt{ }(-\Sigma)}=\mathrm{sn}^{-1} \sqrt{\frac{\sigma-s_{2}}{\sigma-s_{3}}}, \quad \mathrm{sn}^{-1} \sqrt{\frac{s_{3}-\sigma}{s_{2}-\sigma}}
$$

3. Besides the first elliptic integral (I. E. I.), given already in $\S 2(1),(2)$, (11), (12), (13), (14), we shall require the second elliptic integral (II. E. I.) complete and incomplete.

The jacobian zeta function which expresses the incomplete II. E. I. may be efined by the standard integral

$$
\int_{s_{3}}^{s} \frac{s_{1}-s}{\sqrt{\left(s_{1}-s_{3}\right)}} \frac{d s}{\sqrt{S}}=\int_{0}^{h} \mathrm{dn}^{2} h K \cdot K d h=E \operatorname{am} h K=h H+\mathrm{zn} h K,
$$

or

(2) $\int_{s_{2}}^{\sigma} \frac{\sigma-s_{3}}{\sqrt{\left(s_{1}-s_{3}\right)}} \frac{d \sigma}{\sqrt{(-\Sigma)}}=\int_{0}^{f} \mathrm{dn}^{2} f K^{\prime} \cdot K^{\prime} d f=E \operatorname{am} f K^{\prime}=f H^{\prime}+\operatorname{zn} f K^{\prime}$; 
where zn $u$ in GLAISHER's notation denotes the jacobian function $Z u$; also $H$ and $H^{\prime}$ denote the complete II. E. I. to moduli $k$ and $k^{\prime}$, defined by

$$
H=\int_{0}^{1} \mathrm{dn}^{2} h K \cdot K d h, \quad H^{\prime}=\int_{0}^{1} \mathrm{dn}^{2} f K^{\prime} \cdot K^{\prime} d f,
$$

corresponding to $K$ and $K^{\prime}$, the complete I. E. I.

The letter $H$ is used instead of the usual $E$, as $E$ will be required for the quadric transformation.

With GLAISHER's notation

$$
\text { zs } u=\operatorname{zn} u+\frac{d}{d u} \log \operatorname{sn} u,
$$

the incomplete II. E. I. in the regions

is given by

$$
\infty>s>s_{1}>s_{2}>s>s_{3},
$$

(5) $\int_{s_{1}}^{s} \frac{s-s_{1}}{\sqrt{ }\left(s_{1}-s_{3}\right)} \frac{d s}{\sqrt{ } S}=\int_{s}^{s_{2}} \frac{s_{2}-s}{s-s_{3}} \frac{\sqrt{ }\left(s_{1}-s_{3}\right) d s}{\sqrt{ } S}=-(1-h) H+\mathrm{zs} h K$;

(6) $\int \frac{s-s_{2}}{\sqrt{ }\left(s_{1}-s_{3}\right)} \frac{d s}{\sqrt{ } S}=k^{2} \int \frac{s_{1}-s}{s-s_{3}} \frac{\sqrt{ }\left(s_{1}-s_{3}\right) d s}{\sqrt{ } S}=-(1-h)\left(H-k^{\prime 2} K\right)+\mathrm{zs} h K$;

(7) $\int \frac{s-s_{3}}{\sqrt{ }\left(s_{1}-s_{3}\right)} \frac{d s}{\sqrt{ } S}=\int \frac{s_{2}-s_{3}}{s-s_{3}} \frac{V\left(s_{1}-s_{3}\right) d s}{\sqrt{ } S}=(1-h)(K-H)+\mathrm{zs} h K$;

the integrals being infinite at the upper limit $s=\infty$, or the lower limit $s=s_{3}$, where $h=0$ and zs $h K$ is infinite; and so also

(8) $\int_{s, s_{1}}^{\infty, s} \frac{s-s_{2}}{s-s_{3}} \frac{V\left(s_{1}-s_{3}\right) d s}{\sqrt{ } S}=\int_{s_{3}, s}^{s, s_{2}} \frac{s_{1}-s}{\sqrt{ }\left(s_{1}-s_{3}\right)} \frac{d s}{\sqrt{ } S}$

$$
=h H+\mathrm{zn} h K,(1-h) H-\mathrm{zn} h K \text {; }
$$

(9)

$$
\begin{aligned}
\int \frac{s-s_{1}}{s-s_{3}} \frac{\sqrt[V]{ }\left(s_{1}-s_{3}\right) d s}{\sqrt{ } S} & =\int \frac{s_{2}-s}{\sqrt{ }\left(s_{1}-s_{3}\right)} \frac{d s}{\sqrt{ } S} \\
& =h\left(H-{k^{\prime}}^{2} K\right)+\mathrm{zn} h K,(1-h)\left(H-{k^{\prime}}^{2} K\right)-\mathrm{zn} h K
\end{aligned}
$$

$$
\begin{aligned}
\int \frac{s_{2}-s_{3}}{s-s_{3}} \frac{\sqrt{ }\left(s_{1}-s_{3}\right) d s}{\sqrt{ } S} & =\int \frac{s-s_{3}}{\sqrt{ }\left(s_{1}-s_{3}\right)} \frac{d s}{\sqrt{ } S} \\
& =h(K-H)-\operatorname{zn} h K,(1-h)(K-H)+\operatorname{zn} h K
\end{aligned}
$$

Similarly, in the regions

$$
s_{1}>\sigma>s_{2}>s_{3}>\sigma>-\infty,
$$


(11) $\int_{\sigma, s_{2}}^{s_{1}, \sigma} \frac{s_{1}-\sigma}{\sqrt{ }\left(s_{1}-s_{3}\right)} \frac{d \sigma}{V(-\Sigma)}=\int_{-\infty, \sigma}^{\sigma, s_{3}} \frac{s_{1}-s_{2}}{s_{1}-\sigma} \frac{V\left(s_{1}-s_{3}\right) d \sigma}{V(-\Sigma)}$

$$
=f\left(K^{\prime}-H^{\prime}\right)-\mathrm{zn} f K^{\prime},(1-f)\left(K^{\prime}-H^{\prime}\right)+\mathrm{zn} f K^{\prime} ;
$$

$$
\begin{aligned}
\int \frac{\sigma-s_{2}}{\sqrt{ }\left(s_{1}-s_{3}\right)} \frac{d \sigma}{\sqrt{ }(-\Sigma)} & =k^{\prime 2} \int \frac{s_{3}-\sigma}{s_{1}-\sigma} \frac{\sqrt{ }\left(s_{1}-s_{3}\right) d \sigma}{\sqrt{ }(-\Sigma)} \\
& =f\left(H^{\prime}-k^{2} K^{\prime}\right)+\operatorname{zn} f K^{\prime},(1-f)\left(H^{\prime}-k^{2} K^{\prime}\right)-\operatorname{zn} f K^{\prime}
\end{aligned}
$$

$$
\begin{aligned}
\int \frac{\sigma-s_{3}}{\sqrt{ }\left(s_{1}-s_{3}\right)} \frac{d \sigma}{\sqrt{ }(-\Sigma)} & =\int \frac{s_{2}-\sigma}{s_{1}-\sigma} \frac{V\left(s_{1}-s_{3}\right) d \sigma}{V(-\Sigma)} \\
& =f H^{\prime}+\operatorname{zn} f K^{\prime},(1-f) H^{\prime}-\operatorname{zn} f K^{\prime}
\end{aligned}
$$

$$
\begin{aligned}
\int_{s_{2}}^{\sigma} \frac{s_{1}-s_{2}}{s_{1}-\sigma} \frac{V\left(s_{1}-s_{3}\right) d \sigma}{\sqrt{ }(-\Sigma)} & =\int_{\sigma}^{s_{3}} \frac{s_{1}-\sigma}{\sqrt{ }\left(s_{1}-s_{3}\right)} \frac{d \sigma}{\sqrt{ }(-\Sigma)} \\
& =(1-f)\left(K^{\prime}-H^{\prime}\right)+\mathrm{zs} f K^{\prime} \\
k^{\prime 2} \int \frac{s_{3}-\sigma}{s_{1}-\sigma} \frac{\sqrt{ }\left(s_{1}-s_{3}\right) d \sigma}{V(-\Sigma)} & =\int \frac{s_{2}-\sigma}{V\left(s_{1}-s_{3}\right)} \frac{d \sigma}{\sqrt{ }(-\Sigma)} \\
& =-(1-f)\left(H^{\prime}-k^{2} K^{\prime}\right)+\mathrm{zs} f K^{\prime} \\
\int \frac{s_{2}-\sigma}{s_{1}-\sigma} \frac{\sqrt{ }\left(s_{1}-s_{3}\right) d \sigma}{V(-\Sigma)} & =\int \frac{s_{3}-\sigma}{\sqrt{ }\left(s_{1}-s_{3}\right)} \frac{d \sigma}{\sqrt{ }(-\Sigma)} \\
& =-(1-f) H^{\prime}+\mathrm{zs} f K^{\prime}
\end{aligned}
$$

these integrals (14), (15), (16) heing infinite when the upper limit $\sigma=s_{1}$, or the lower limit $\sigma=-\infty$, where $f=0$ and $\mathrm{zs} f K^{\prime}$ is infinite.

Putting $h=1$ or $f=1$ in any of these forms will give the corresponding complete II. E. I., noticing that $\mathrm{zn} K^{\prime}$ and $\mathrm{zs} K^{\prime}$ are zero.

4. The incomplete III. E. I. requires the theta and eta functions of J ACOBI, and BurKHARDT has given in his Elliptische Functionen, $\$ 126$, a method of approximating rapidly to the numerical value for given elliptic argument.

But in the circular III. E. I. this argument will be complex of the form $h K+f K^{\prime} i$, where $h$ and $f$ are real fractions, so that great care will be required with BURKHARDT's method in separating the real and imaginary parts of the theta function.

When $f$, however, is a rational fraction,

$$
f=\frac{1}{2}, \frac{1}{3}, \frac{1}{4}, \frac{1}{6}, \cdots,
$$

a quotient of theta functions, such as 


$$
\frac{\Theta\left(h K+f K^{\prime} i\right)}{\Theta\left(h K-f K^{\prime} i\right)}, \quad \frac{\Theta 0 \Theta\left(h K+f K^{\prime} i\right)}{\Theta h K \Theta f K^{\prime} i},
$$

can be expressed algebraically by elliptic functions of $h K$; a collection of the simplest results will be found in the Philosophical Transactions (1904), under the title, The third elliptic integral and the ellipsotomic problem.

5. Make a start now with the complete circular III. E. I.

$$
A, B=\int_{s_{1}, s_{8}}^{\infty, s_{2}} \frac{\frac{1}{2} \sqrt{ }(-\Sigma)}{s \sim \sigma} \frac{d s}{\sqrt{ } S},
$$

in which the quantities range in the order of magnitude

$$
\infty>s>s_{1}>\sigma>s_{2}>s>s_{3}>-\infty
$$

and this integral (1) is by LEGENDRE's theorem to be expressed by incomplete integrals, I. E. I. and II. E. I.

Provided with the notation above we shall find in the sequel

$$
\begin{gathered}
A=\int_{s_{1}}^{\infty} \frac{\frac{1}{2} V(-\Sigma)}{s-\sigma} \frac{d s}{\sqrt{S}}=\frac{1}{2} \pi(1-f)-K \mathrm{zn} f K^{\prime}, \\
B=\int_{s_{3}}^{s_{2}} \frac{\frac{1}{2} V(-\Sigma)}{\sigma-s} \frac{d s}{\sqrt{ } S}=\frac{1}{2} \pi f+K \mathrm{zn} f K^{\prime}, \\
A+B=\frac{1}{2} \pi .
\end{gathered}
$$

To calculate the numerical value we have to determine the co-modular angle $\theta$ and the amplitude angle of $\phi$ from the relations (2) and (8), § 2,

$$
\sin ^{2} \theta=k^{2}=\frac{s_{1}-s_{2}}{s_{1}-s_{3}}, \quad \sin ^{2} \phi=\frac{s_{1}-\sigma}{s_{1}-s_{2}},
$$

and then from Table IX of LEGENDRE

$$
\begin{aligned}
f & =F \phi \div F\left(\frac{1}{2} \pi\right), \\
\text { zn } f K^{\prime} & =E \phi-f E\left(\frac{1}{2} \pi\right), \\
K & =F\left(\frac{1}{2} \pi, k\right) .
\end{aligned}
$$

The result in the Weierstrassian notation is given in Schwarz's Formeln, $\S \S 59,60$.

The relation in (5) is the equivalent of Minchis's equation (21), p. 212, Philosophical Magazine, February, 1894 ; it is proved immediately by the substitution 


$$
\left(s-s_{3}, \frac{s_{1}-s_{3} \cdot s_{2}-s_{3}}{s-s_{3}}\right)
$$

in $A,(1)$, which leaves $d s / \sqrt{ } S$ unaltered except for sign, but changes the limits from $\infty$ and $s_{1}$ to $s_{3}$ and $s_{2}$, and thus makes

$$
A=\int_{s_{3}}^{s_{2}} \frac{\frac{1}{2} V(-\Sigma)}{\frac{s_{1}-s_{3} \cdot s_{2}-s_{3}}{s-s_{3}}-\sigma+s_{3}} \frac{d s}{\sqrt{S}},
$$

$$
\begin{aligned}
A+B & =\int \frac{\frac{1}{2} V(-\Sigma)\left[\left(s_{1}-s_{3}\right)\left(s_{2}-s_{3}\right)-\left(s-s_{3}\right)^{2}\right]}{\left[\left(s_{1}-s_{3} \cdot s_{2}-s_{3}\right)-\left(s-s_{3} \cdot \sigma-s_{3}\right)\right](\sigma-s)} \frac{d s}{\dot{V} S} \\
& =\cos ^{-1} \sqrt{\frac{\sigma-s_{3} \cdot s_{1}-s \cdot s_{2}-s}{\left[\left(s_{1}-s_{3}\right)\left(s_{2}-s_{3}\right)-\left(s-s_{3}\right)\left(\sigma-s_{3}\right)\right](\sigma-s)}} \\
& \left.=\sin ^{-1} \sqrt{\frac{s_{1}-\sigma \cdot \sigma-s_{2} \cdot s-s_{3}}{\left[\left(s_{1}-s_{3} \cdot s_{2}-s_{3}\right)-\left(s-s_{3}\right)\left(\sigma-s_{3}\right)\right](\sigma-s)}}\right]_{s_{3}}^{s_{2}}=\frac{1}{2} \pi .
\end{aligned}
$$

6. In the arrangement

$$
\infty>s>s_{1}>s_{2}>s>s_{3}>\sigma>-\infty,
$$

there are two remaining forms of the complete circular III. E. I., namely

and we shall find

$$
C, D=\int_{s_{1}, s_{8}}^{\infty, s_{1}} \frac{\frac{1}{2} \sqrt{ }(-\Sigma)}{s-\sigma} \frac{d s}{\sqrt{S}}
$$

$$
\begin{aligned}
& C=\int_{s_{1}}^{\infty} \frac{\frac{1}{2} \sqrt{ }(-\Sigma)}{s-\sigma} \frac{d s}{\sqrt{S}}=K \mathrm{zs} f K^{\prime}-\frac{1}{2} \pi(1-f), \\
& D=\int_{s_{3}}^{s_{2}} \frac{\frac{1}{2} \sqrt{ }(-\Sigma)}{s-\sigma} \frac{d s}{\sqrt{ } S}=K \mathrm{zs} f K^{\prime}+\frac{1}{2} \pi f,
\end{aligned}
$$

where in Glaisher's notation and also

$$
\text { zs } u=\operatorname{zn} u+\frac{\operatorname{cn} u \operatorname{dn} u}{\operatorname{sn} u}=\operatorname{zn} u+\frac{d}{d u} \log \operatorname{sn} u=\frac{d}{d u} \log H u,
$$

$$
\begin{aligned}
& \operatorname{zc} u=\operatorname{zn} u+\frac{d}{d u} \log \operatorname{cn} u=-\operatorname{zs}(K-u), \\
& \operatorname{zd} u=\operatorname{zn} u+\frac{d}{d u} \log \operatorname{dn} u=-\operatorname{zn}(K-u),
\end{aligned}
$$




$$
-C+D=\frac{1}{2} \pi
$$

which can be proved independently in the same manner as $(12) \S 5$.

Hence we can write down the expression of the complete III. E. I. of the circular form, for any assigned region of $s$ and $\sigma$, the result involving a zeta function to the complementary modulus; and this zeta function is an algebraical function when $f$ is a rational fraction; the formula for the simplest fractions is given in the Philosophical Transactions (1904).

7. We have seen how $A$ and $B$ change place by the substitution

$$
\left(s-s_{3}, \frac{s_{1}-s_{3} \cdot s_{2}-s_{3}}{s-s_{3}}\right),
$$

and the same substitution interchanges $C$ and $D$.

So also $A$ and $C$ or $B$ and $D$ change place by the substitution

while the substitution

$$
\begin{aligned}
& \left(s-s_{1}, \frac{s_{1}-s_{2} \cdot s_{1}-s_{3}}{s-s_{1}}\right) ; \\
& \left(s-s_{2}, \frac{s_{2}-s_{1} \cdot s_{2}-s_{3}}{s-s_{2}}\right)
\end{aligned}
$$

interchanges $A$ and $D$, or $B$ and $C$.

8. Two more forms are required to finish the series of the complete III. E. I., when it is finite and the parameter is a fraction of the real period, namely,

in the arrangement

$$
\begin{aligned}
& E=\int_{s_{1}}^{\infty} \frac{\frac{1}{2} V \Sigma}{s-\sigma} \frac{d s}{\sqrt{S}}=K \mathrm{zn} f K, \\
& F=\int_{s_{3}}^{s_{2}} \frac{\frac{1}{2} V}{s-\sigma} \frac{S}{\sqrt{\Sigma}}=K \mathrm{zs} h K,
\end{aligned}
$$

with

$$
\infty>s>s_{1}>s_{2}>\sigma>s_{3}>-\infty,
$$

$$
\begin{aligned}
& h=\int_{s}^{\infty} \div \int_{s_{1}}^{\infty} \frac{d s}{\sqrt{S}}, \\
& f=\int_{s_{3}}^{s} \div \int_{s_{3}}^{s_{2}} \frac{d \sigma}{\sqrt{ }} .
\end{aligned}
$$

(Legendre, Fonctions elliptiques, chap. 23 ; CaYley, Elliptic Functions, $\S \S 178-186$.

9. The proof of his theorem that the complete III. E. I., circular or logarithmic, can be expressed by the I. and II. E. I. is given by LEGENDRE in Fonctions elliptiques, chapters 23 and 24, and is reproduced in CAYLEY's Elliptic 
Functions, chap. 5. We can abbreviate the demonstration considerably, and avoid also all introduction of an imaginary in the discussion of the circular form expressed by $A, B C$, and $D$ by means of the simple lemma

$$
\checkmark S \frac{d}{d s} \frac{\frac{1}{2} \vee S}{s \sim \sigma}-\sqrt{ }(-\Sigma) \frac{d}{d \sigma} \frac{\frac{1}{2} *(-\Sigma)}{s \sim \sigma}=s \sim \sigma,
$$

proved immediately by performing the differentiation.

Integrating with respect to the elliptic differential elements $d s / \sqrt{ } S$ and $d \sigma / V(-\Sigma)$ beween the limits $s_{3}, s_{2}$ of $s$, and $\sigma, s_{1}$ of $\sigma$,

$$
\begin{aligned}
\iint \frac{d \sigma}{\sqrt{ }(-\Sigma)} & \frac{d s}{\sqrt{ } S^{y}} \sqrt{ } S \frac{d}{d s} \frac{\frac{1}{2} \sqrt{ } S}{\sigma-s}-\iint \frac{d s}{\sqrt{ } S} \frac{d \sigma}{\sqrt{ }(-\Sigma)} \sqrt{ }(-\Sigma) \frac{d}{d c} \frac{\frac{1}{2} \sqrt{ }(-\Sigma)}{-s} \\
& =\int_{\sigma}^{s_{1}}\left(\frac{\frac{1}{2} \sqrt{ } S}{\sigma-s}\right)_{s_{8}}^{s_{3}} \frac{d \sigma}{\sqrt{ }(-\Sigma)}(\text { or } 0)+\int_{s_{3}}^{s_{2}} \frac{\frac{1}{2} \sqrt{ }(-\Sigma)}{\sigma-s} \frac{d s}{\sqrt{ } S}(\text { or } B) \\
& =\iint(\sigma-s) \frac{d s}{\sqrt{S}} \frac{d \sigma}{\sqrt{ }(-\Sigma)},
\end{aligned}
$$

in which the variables are separated, so that

$$
\begin{aligned}
B & =\int_{\sigma}^{s_{1}} \int_{s_{8}}^{s_{3}}\left(\sigma-s_{3}\right) \frac{d s}{\sqrt{S}} \frac{d \sigma}{\sqrt{ }(-\Sigma)}-\iint\left(s-s_{3}\right) \frac{d s}{\sqrt{ } S} \frac{d \sigma}{\sqrt{ }(-\Sigma)} \\
& =K \int_{\sigma}^{s_{1}} \frac{\sigma-s_{3}}{\sqrt{\left(s_{1}-s_{3}\right)}} \frac{d \sigma}{\sqrt{ }(-\Sigma)}-f K^{\prime} \int_{s_{3}}^{s_{2}} \frac{s-s_{3}}{\sqrt{ }\left(s_{1}-s_{3}\right)} \frac{d s}{\sqrt{ } S} \\
& =K\left(f H^{\prime}+\mathrm{zn} f K^{\prime}\right)-f K^{\prime}(K-H) \\
& =\frac{1}{2} \pi f+K \mathrm{zn} f K^{\prime},
\end{aligned}
$$

by reason of LEGENDRE's relation

$$
\int_{s_{2}}^{s_{1}} \int_{s_{8}}^{s_{3}}(\sigma-s) \frac{d s}{\sqrt{S}} \frac{d \sigma}{\sqrt{ }(-\Sigma)}=K H^{\prime}+K^{\prime} H-K K^{\prime}=\frac{1}{2} \pi,
$$

derivable from the relation in (5) $\S 5$, and utilising theorems (13) and (10), 33 .

Taking the limits of $s$ as $s_{3}$ and $s$ gives a more general theorem

$$
\begin{aligned}
\int_{\sigma}^{s_{1}} \frac{\frac{1}{2} \sqrt{ } S}{\sigma-s} & \frac{d \sigma}{\sqrt{ }(-\Sigma)}+\int_{s_{3}}^{s} \frac{\frac{1}{2} V(-\Sigma)}{\sigma-s} \frac{d s}{\sqrt{S}} \\
& =h K \int_{\sigma}^{s_{1}} \frac{\sigma-s_{3}}{\sqrt{\left(s_{1}-s_{3}\right)}} \frac{d \sigma}{\sqrt{ }(-\Sigma)}-f K^{\prime} \int_{s_{s}}^{s} \frac{s-s_{3}}{\sqrt{ }\left(s_{1}-s_{3}\right)} \frac{d s}{\sqrt{ } S} \\
& =h K\left(f H^{\prime}+\mathrm{zn} f K^{\prime}\right)-f K^{\prime}[h(K-H)-\mathrm{zn} h K] \\
& =\frac{1}{2} \pi h f+h K \mathrm{zn} f K^{\prime}-f K^{\prime} \mathrm{zn} h K,
\end{aligned}
$$


connecting incomplete III. E.I's., as in LEGENDRE's equation $\left(i^{\prime}\right)$, p. 133.

Putting $h=1$ gives $B$ as before; and putting $f=1$ leads to a new theorem

$$
\int_{s_{2}}^{s_{1}} \frac{1}{2} \sqrt{ } S \frac{d \sigma}{\sigma-s} \frac{1}{V(-\Sigma)}=\frac{1}{2} \pi h-K^{\prime} \operatorname{zn} h K .
$$

10. When $A$ is deduced in this manner we must keep clear of $s=\infty$ by writing the lemma $(1), \S 9$, in the form

(1) $\sqrt{ }(-\Sigma) \frac{d}{d \sigma} \frac{\frac{1}{2} V(-\Sigma)}{s-\sigma}-\sqrt{ } S \frac{d}{d s}\left(\frac{\frac{1}{2} \sqrt{ } S}{s-\sigma}-\frac{\frac{1}{2} \sqrt{ } S}{s-s_{3}}\right)=\sigma-s_{3}-\frac{s_{1}-s_{3} \cdot s_{2}-s_{3}}{s-s_{3}}$, in which at

$$
s=\infty, \quad \operatorname{limit}\left(\frac{\frac{1}{2} \sqrt{ } S}{s-\sigma}-\frac{\frac{1}{2} \vee S}{s-s_{3}}\right)=0
$$

Integrating between the limits $s, \infty$ of $s$, and $s_{2}, \sigma$ of $\sigma$, we have

$$
\begin{aligned}
& \int_{s}^{\infty} \frac{\frac{1}{2} V(-\Sigma)}{s-\sigma} \frac{d s}{\sqrt{ } S}+\int_{s_{s}}^{\sigma}\left(\frac{\frac{1}{2} V S}{s-\sigma}-\frac{\frac{1}{2} V S}{s-s_{3}}\right) \frac{d \sigma}{\sqrt{ }(-\Sigma)} \\
& =\iint\left(\sigma-s_{3}-\frac{s_{1}-s_{3} \cdot s_{2}-s_{3}}{s-s_{3}}\right) \frac{d s}{\sqrt{ } S} \frac{d \sigma}{V(-\Sigma)} \\
& \text { (in which the variables are separated) } \\
& =h K \int_{x_{2}}^{\sigma} \frac{\sigma-s_{3}}{\sqrt{ }\left(s_{1}-s_{3}\right)} \frac{d \sigma}{V(-\Sigma)}-(1-f) K^{\prime} \int_{s}^{\infty} \frac{s_{2}-s_{3}}{s-s_{3}} \frac{V\left(s_{1}-s_{3}\right) d s}{V S} \\
& =h K\left[(1-f) H^{\prime}-\operatorname{zn} f K^{\prime}\right]-(1-f) K^{\prime}[h(K-H)-\operatorname{zn} h K] \\
& =\frac{1}{2} \pi h(1-f)-h K \mathrm{zn} f K^{\prime}+(1-f) K^{\prime} \mathrm{zn} h K,
\end{aligned}
$$

utilising the theorems in (13) and (5), § 3 .

Putting $s=s_{1}, h=1$, gives the result for $A$,

(4) $A=\int_{s_{1}}^{\infty} \frac{\frac{1}{2} V(-\Sigma)}{s-\sigma} \frac{d s}{\sqrt{ } S}=\int_{s_{1}}^{\infty} \int_{s_{2}}^{\sigma}\left(\sigma-s_{3}-\frac{s_{1}-s_{3} \cdot s_{2}-s_{3}}{s-s_{3}}\right) \frac{d \sigma}{V(-\Sigma)} \frac{d s}{V S}$,

and this by the substitution $(10), \S 5$, is equivalent to

while from $(2), \S 9$,

$$
A=\int_{s_{3}}^{\sigma} \int_{s_{3}}^{s_{2}}(\sigma-s) \frac{d s}{\sqrt{S}} \frac{d \sigma}{\sqrt{(-\Sigma)}},
$$

$$
B=\int_{\sigma}^{s_{1}} \int_{s_{3}}^{s_{2}}(\sigma-s) \frac{d s}{V S} \frac{d \sigma}{\sqrt{ }(-\Sigma)},
$$




$$
A+B=\int_{s_{2}}^{s_{1}} \int_{s_{3}}^{s_{2}}(\sigma-s) \frac{d s}{\sqrt{S}} \frac{d \sigma}{\sqrt{ }(-\Sigma)}
$$

or from (12), §5, and employing (12), (9), § 3,

$$
\begin{aligned}
\frac{1}{2} \pi & =\iint\left[\sigma-s_{2}+s_{2}-s\right] \frac{d s}{\sqrt{S}} \frac{d \sigma}{\sqrt{(-\Sigma)}} \\
& =K\left(H^{\prime}-k^{2} K^{\prime}\right)+K^{\prime}\left(H-{k^{\prime}}^{2} K\right) ;
\end{aligned}
$$

and thus LEGENDRE's relation is proved.

Putting $f=0, \sigma=s_{1}$ in (3) gives a new theorem,

(9) $\int_{s_{2}}^{s_{1}} \frac{\frac{1}{2} V S}{s-\sigma} \frac{d \sigma}{V(-\Sigma)}=\frac{1}{2} \pi h+K^{\prime}$ zn $h K+K^{\prime} \sqrt{\frac{s-s_{1} \cdot s-s_{2}}{s-s_{3} \cdot s_{1}-s_{3}}}=\frac{1}{2} \pi h+K^{\prime}$ zs $h K$, employing (5), (6), (7), § 2, and (4), §3, or (5), §6.

11. For $C$, integrate lemma (1), $\S 9$, from $s$ to $\infty$ and $\sigma$ to $s_{3}$ in the form

(1) $-\vee(-\Sigma) \frac{d}{d \sigma} \frac{\frac{1}{2} \vee(-\Sigma)}{s-\sigma}+\vee S \frac{d}{d s}\left(\frac{\frac{1}{2} v S}{s-\sigma}-\frac{\frac{1}{2} \vee S}{s-s_{3}}\right)=s_{3}-\sigma+\frac{s_{1}-s_{3} \cdot s_{2}-s_{3}}{s-s_{3}}$,

$$
\begin{aligned}
\int_{s}^{\infty} \frac{\frac{1}{2} V}{s}-\sigma & \frac{d s}{\sqrt{ }}-\int_{\sigma}^{s_{3}}\left(\frac{\frac{1}{2} V S}{s-\sigma}-\frac{\frac{1}{2} V S}{s-s_{3}}\right) \frac{d \sigma}{V(-\Sigma)} \\
& =\iint\left(s_{3}-\sigma+\frac{s_{1}-s_{3} \cdot s_{2}-s_{3}}{s-s_{3}}\right) \frac{d s}{\sqrt{ } S} \frac{d \sigma}{\sqrt{ }(-\Sigma)} \\
& =h K \int_{\sigma}^{s_{3}} \frac{s_{3}-\sigma}{V\left(s_{1}-s_{3}\right)} \frac{d \sigma}{V(-\Sigma)}+(1-f) K^{\prime} \int_{s}^{\infty} \frac{s_{2}-s_{3}}{s-s_{3}} \frac{v^{\prime}\left(s_{1}-s_{3}\right)}{V S} \\
& =h K\left[\mathrm{zs} f K^{\prime}-(1-f) H^{\prime}\right]+(1-f) K^{\prime}[h(K-H)-\mathrm{zn} h K] \\
& =-\frac{1}{2} \pi h(1-f)-(1-f) K^{\prime} \mathrm{zn} h K+h K \mathrm{zs} f K^{\prime},
\end{aligned}
$$

employing (16), (10), $\S 3$; from which $C$ is deduced by putting $h=1, s=s_{1}$,

$$
C=-\frac{1}{2} \pi(1-f)+K \mathrm{zs} f K^{\prime} .
$$

In $D$ we must keep clear of $\sigma=s_{1}$ by writing the lemma

(4) $\vee(-\Sigma) \frac{d}{d \sigma}\left(\frac{\frac{1}{2} V(-\Sigma)}{s-\sigma}-\frac{\frac{1}{2} V(-\Sigma)}{s_{1}-\sigma}\right)-\vee S \frac{d}{d s} \cdot \frac{\frac{1}{2} V S}{s-\sigma}=s_{1}-s-\frac{s_{1}-s_{2} \cdot s_{1}-s_{3}}{s_{1}-\sigma}$ and then integrate it between $s_{3}$ and $s,-\infty$ and $\sigma$, so that

$$
\int_{s_{3}}^{s}\left(\frac{\frac{1}{2} v^{\prime}(-\Sigma)}{s-\sigma}-\frac{\frac{1}{2} V(-\Sigma)}{s_{1}-\sigma}\right) \frac{d s}{V S}-\int_{-\infty}^{\sigma} \frac{\frac{1}{2} V S}{s-\sigma} \frac{d \sigma}{V(-\Sigma)}
$$




$$
\begin{aligned}
& =f K^{\prime} \int \frac{s_{1}-s}{\sqrt{\left(s_{1}-s_{3}\right)}} \frac{d s}{\sqrt{S}}-h K \int \frac{s_{1}-s_{2}}{s_{1}-\sigma} \sqrt{ }\left(s_{1}-s_{3}\right) \frac{d \sigma}{\sqrt{ }(-\Sigma)} \\
& =f K^{\prime}(h H+\operatorname{zn} h K)-h K\left[f\left(K^{\prime}-H^{\prime}\right)-\operatorname{zn} f K^{\prime}\right] \\
& =\frac{1}{2} \pi h f+f K^{\prime} \operatorname{zn} h K+h K \operatorname{zn} f K^{\prime},
\end{aligned}
$$

employing (8), (11), § 3 .

Put $h=1, s=s_{2}$, and we obtain by means of (8), (9), (10), 2 ,

(6) $D=\frac{1}{2} \pi f+K \mathrm{zn} f K^{\prime}+K \sqrt{\frac{\sigma-s_{2} \cdot \sigma-s_{3}}{s_{1}-\sigma \cdot s_{1}-s_{3}}}=\frac{1}{2} \pi f+K \mathrm{zs} f K^{\prime}$.

12. To obtain $E$ and $F$, write the lemma

$$
\sqrt{ } \Sigma \frac{d}{d \sigma} \frac{\frac{1}{2} \sqrt{ } \Sigma}{s-\sigma}+\sqrt{ } S \frac{d}{d s}\left(\frac{\frac{1}{2} \sqrt{ } S}{s-\sigma}-\frac{\frac{1}{2} V S}{s-s_{3}}\right)=\frac{s_{1}-s_{3} \cdot s_{2}-s_{3}}{s-s_{3}}-\sigma+s_{3},
$$

and integrate between $s, \infty$ and $s_{3}, \sigma$; then

$$
\begin{aligned}
& \int_{s}^{\infty} \frac{\frac{1}{2} \sqrt{ } \Sigma}{s-\sigma} \frac{d s}{\sqrt{ } S}-\int_{s_{3}}^{\sigma}\left(\frac{\frac{1}{2} V}{s-\sigma}-\frac{\frac{1}{2} v^{\prime}}{s-s_{3}}\right) \frac{d \sigma}{\sqrt{ } \Sigma} \\
& =f K \int \frac{s_{2}-s_{3}}{s-s_{3}} \frac{v^{\prime}\left(s_{1}-s_{3}\right) d s}{V S}-h K \int \frac{\sigma-s_{3}}{V\left(s_{1}-s_{3}\right)} \frac{d \sigma}{\sqrt{ }} \bar{\Sigma} \\
& =f K[h(K-H)-\mathbf{z n} h K]-h K[f(K-H)-\mathbf{z n} f K] \\
& =h K \mathrm{zn} f K-f K \mathrm{zn} h K,
\end{aligned}
$$

employing (10), $\S 3$, and thus connecting $E$ and $F$; for $h=1, s=s_{1}$, makes

and $f=1, \sigma=s_{2}$, makes

$$
E=K \mathrm{zn} f K,
$$

$$
F=K \mathrm{zn} h K+K \sqrt{\frac{s-s_{1} \cdot s-s_{2}}{s-s_{3} \cdot s_{1}-s_{3}}}=K \mathrm{zs} h K .
$$

13. With $s_{1}$ and $s_{3}$ conjugate imaginary, and putting

and then

$$
\begin{gathered}
s_{1}-s_{2} \cdot s_{3}-s_{2}=M^{2}, \\
K=\int_{s_{2}}^{\infty} \frac{\sqrt{ } M d s}{\sqrt{ } S}, \quad h K=\int_{s_{2}}^{s} \frac{\sqrt{ } M d s}{\sqrt{ }}, \\
K^{\prime}=\int_{-\infty}^{s_{2}} \frac{V M d \sigma}{\sqrt{ }(-\Sigma)}, \quad f K^{\prime}=\int_{\sigma}^{s_{2}} \frac{\sqrt{ } M d \sigma}{\sqrt{ }(-\Sigma)},
\end{gathered}
$$

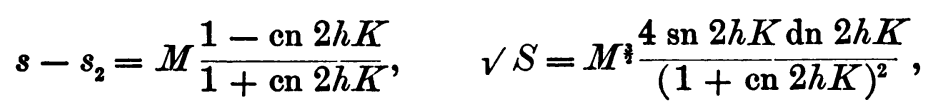

Trans. Am. Math. Soc. 31 
(5) $\quad s_{2}-\sigma=M \frac{1-\operatorname{cn} 2 f K}{1+\operatorname{cn} 2 f K}, \quad V(-\Sigma)=M^{3} \frac{4 \operatorname{sn} 2 f K^{\prime} \operatorname{dn} 2 f K^{\prime}}{\left(1+\operatorname{cn} 2 f K^{\prime}\right)^{2}}$,

in which $h K$ and $f K^{\prime}$ may be replaced occasionally by $h$ and $f$ in the sequel without confusion.

Employing a formula (LEgendre I, p. 257),

$$
\int \frac{1-\cos \phi}{1+\cos \phi} \frac{d \phi}{\Delta \phi}=\frac{2 \sin \phi \Delta \phi}{1+\cos \phi}+F \phi-2 E \phi,
$$

verified immediately by differentiation, and integrating the lemma (1) §9,

$$
\begin{aligned}
\int_{\sigma}^{s_{2}} \frac{\frac{1}{2} V S}{s-\sigma} & \frac{d \sigma}{V(-\Sigma)}+\int_{s_{2}}^{s} \frac{\frac{1}{2}(V-\Sigma)}{s-\sigma} \frac{d s}{\sqrt{ } S}=\int_{\sigma}^{s_{2}} \int_{s_{2}}^{s}(s-\sigma) \frac{d s}{V S} \frac{d \sigma}{\sqrt{ }(-\Sigma)} \\
& =f K^{\prime} \int_{s_{2}}^{s} \frac{s-s_{2}}{M} \cdot \frac{V M d s}{V S}+h K \int_{\sigma}^{s_{3}} \frac{s_{2}-\sigma}{M} \frac{V M d \sigma}{V(-\Sigma)} \\
& =f K^{\prime} \int_{0}^{h} \frac{1-\operatorname{cn} 2 h}{1+\operatorname{cn} 2 h} K d h+h K \int_{0}^{f} \frac{1-\operatorname{cn} 2 f}{1+\operatorname{cn} 2 f} K^{\prime} d f \\
& =f K^{\prime}\left(\frac{\operatorname{sn} 2 h \operatorname{dn} 2 h}{1+\operatorname{cn} 2 h}+\frac{1}{2} h K-h H-\operatorname{zn} 2 h\right) \\
& +h K\left(\frac{\operatorname{sn} 2 f \operatorname{dn} 2 f}{1+\operatorname{cn} 2 f}+\frac{1}{2} f K^{\prime}-f H^{\prime}-\operatorname{zn} 2 f\right) .
\end{aligned}
$$

But when we wish to obtain the complete III. E. I., by putting $s=\infty$, $h=1$, we make both sides infinite.

The infinite part must then be cut out by deducting from both sides

and now

$$
\int_{\sigma}^{s_{2}} \frac{\frac{1}{2} \sqrt{ } S}{s-s_{2}} \frac{d \sigma}{\sqrt{(-\Sigma)}}=2 f K^{\prime} \frac{\operatorname{dn} 2 h}{\operatorname{sn} 2 h},
$$

$$
\begin{array}{r}
\int_{\sigma}^{s}\left(\frac{\frac{1}{2} V S}{s-\sigma}-\frac{\frac{1}{2} V}{s-\frac{S}{s_{2}}}\right) \frac{d \sigma}{V(-\Sigma)}+\int_{s_{2}}^{s} \frac{\frac{1}{2} V(-\Sigma)}{s-\sigma} \frac{d s}{\sqrt{S}} \\
=f K^{\prime}\left(\frac{-\operatorname{sn} 2 h \operatorname{dn} 2 h}{1-\operatorname{cn} 2 h}+\frac{1}{2} h K-h H-\operatorname{mn} 2 h\right) \\
+h K\left(\frac{\operatorname{sn} 2 f \operatorname{dn} 2 f}{1+\operatorname{cn} 2 f}+\frac{1}{2} f K^{\prime}-f H^{\prime}-\operatorname{mn} 2 f\right) .
\end{array}
$$

Here, putting $s=\infty, h=1$, the first integral vanishes, and

$$
\begin{aligned}
\int_{s_{2}}^{\infty} \frac{\frac{1}{2} V}{s-\sigma} \frac{(-\Sigma)}{\sqrt{S}}=f K^{\prime}\left(\frac{1}{2} K-H\right) & \\
& +K\left(\frac{\left.\operatorname{sn} 2 f \frac{\operatorname{dn} 2 f}{1+\operatorname{cn} 2 f}+\frac{1}{2} f K^{\prime}-f H^{\prime}-\operatorname{mn} 2 f\right)}{}\right.
\end{aligned}
$$




$$
\begin{aligned}
& =K\left(\frac{\operatorname{sn} 2 f \operatorname{dn} 2 f}{1+\operatorname{cn} 2 f}-\operatorname{zn} 2 f\right)-\frac{1}{2} \pi f \\
& =K\left(\frac{\operatorname{dn} 2 f}{\operatorname{sn} 2 f}-\operatorname{zs} 2 f\right)-\frac{1}{2} \pi f
\end{aligned}
$$

Similarly with $f=1$,

$$
\int_{-\infty}^{s_{s}} \frac{\frac{1}{2} V S}{s-\sigma} \frac{d \sigma}{\sqrt{ }(-\Sigma)}=K^{\prime}\left(\frac{\operatorname{dn} 2 h}{\operatorname{sn} 2 h}-\mathrm{zs} 2 h\right)-\frac{1}{2} \pi h .
$$

14. To compare $B$ with Legende's standard form of the III. E. I.,

$$
\int_{0}^{x} \frac{1}{1+n \sin ^{2} \chi} \frac{d \chi}{\Delta \chi}, \quad \Delta \chi=V\left(1-k^{2} \sin ^{2} \chi\right),
$$

denoted by $\Pi(n, k, \chi)$ or $\Pi$, when complete, put

$$
s-s_{3}=\left(s_{2}-s_{3}\right) \sin ^{2} \chi, \quad s_{2}-s=\left(s_{2}-s_{3}\right) \cos ^{2} \chi, \quad s_{1}-s=\left(s_{1}-s_{3}\right) \Delta^{2} \chi,
$$

$$
k^{2}=\frac{s_{2}-s_{3}}{s_{1}-s_{3}}, \quad k^{\prime 2}=\frac{s_{1}-s_{2}}{s_{1}-s_{3}} ;
$$

and then

$$
B=\sqrt{\frac{s_{1}-\sigma \cdot \sigma-s_{2}}{s_{1}-s_{3} \cdot \sigma-s_{3}}} \int \frac{1}{1+n \sin ^{2} \chi} \frac{d \chi}{\Delta \chi}=\vee \alpha \Pi(n, k, \chi),
$$

in which, with LEGENDRE's notation,

$$
\begin{gathered}
n=-\frac{s_{2}-s_{3}}{\sigma-s_{3}}, \quad 1+n=\frac{\sigma-s_{2}}{\sigma-s_{3}}, \quad 1+\frac{k^{2}}{n}=\frac{s_{1}-\sigma}{s_{1}-s_{3}}, \\
\alpha=(1+n)\left(1+\frac{k^{2}}{n}\right)=\frac{s_{1}-\sigma \cdot \sigma-s_{2}}{s_{1}-s_{3} \cdot \sigma-s_{3}},
\end{gathered}
$$

and LEGENDRE's $I I$ requires the normalising factor $\sqrt{ } \alpha$.

In the comparison of $A$, put

(6) $s-s_{3}=\frac{s_{1}-s_{3}}{\sin ^{2} \chi}, \quad s-s_{2}=\left(s_{1}-s_{3}\right) \frac{\Delta^{2} \chi}{\sin ^{2} \chi}, \quad s-s_{1}=\left(s_{1}-s_{3}\right) \frac{\cos ^{2} \chi}{\sin ^{2} \chi}$, and then

(7) $A=\sqrt{\frac{s_{1}-\sigma \cdot \sigma-s_{2}}{s_{1}-s_{3} \cdot \sigma-s_{3}}} \int \frac{-n^{\prime} \sin ^{2} \chi}{1+n^{\prime} \sin ^{2} \chi} \frac{d \chi}{\Delta \chi}=\sqrt{ } \alpha\left[\Pi\left(n^{\prime}, k, \chi\right)-F \chi\right]$ in which

so that

$$
n^{\prime}=-\frac{\sigma-s_{3}}{s_{1}-s_{3}}, \quad 1+n^{\prime}=\frac{s_{1}-\sigma}{s_{1}-s_{3}}, \quad 1+\frac{k^{2}}{n^{\prime}}=\frac{\sigma-s_{2}}{\sigma-s_{3}},
$$

$$
\left(1+n^{\prime}\right)\left(1+\frac{k^{2}}{n^{\prime}}\right)=\frac{s_{1}-\sigma \cdot \sigma-s_{2}}{s_{1}-s_{3} \cdot \sigma-s_{3}}=\alpha,
$$


the same as before; also

$$
n n^{\prime}=k^{2}, \quad \Pi(n)+\Pi\left(n^{\prime}\right)=K+\frac{\frac{1}{2} \pi}{\sqrt{ } \alpha} .
$$

But in all the subsequent calculations it is best to keep clear of the standard forms of Legendre, JACOBI, or WeIERSTRASs, and to work with the III. E. I. as the integral of an algebraical function of the standard form in (8) $\S 1$.

For example we find, putting the differential element,

and if we take

$$
\begin{gathered}
\frac{d s}{\sqrt{ } S}=d u, \\
\frac{d^{2} y}{d u^{2}}=\sqrt{ } S \frac{d}{d s}\left(\sqrt{ } S \frac{d y}{d s}\right)=S \frac{d^{2} y}{d s^{2}}+\frac{1}{2} \frac{d S}{d s} \frac{d y}{d s}
\end{gathered}
$$

$$
\log y=\frac{1}{2} \int \frac{\sqrt{ } S-\sqrt{ } \Sigma}{s-\sigma} \frac{d s}{\sqrt{ } S},
$$

we obtain the Lamé differential equation of the second order

$$
\begin{gathered}
\frac{1}{y} \frac{d^{2} y}{d u^{2}}=2 s+\sigma-s_{1}-s_{2}-s_{3}, \\
S \frac{d^{2} y}{d s^{2}}+\frac{1}{2} \frac{d S}{d s} \frac{d y}{d s}-\left(2 s+\sigma-s_{1}-s_{2}-s_{3}\right) y=0 .
\end{gathered}
$$

Then the function

$$
z=\frac{d}{d u}\left(y e^{\lambda u}\right)
$$

leads to the Lamé differential equation of the second order

provided that

$$
\frac{1}{z} \frac{d^{2} z}{d u^{2}}=6 s-3 \sigma-s_{1}-s_{2}-s_{3}+3 \lambda^{2},
$$

$$
\lambda^{3}-\lambda\left(3 \sigma-s_{1}-s_{2}-s_{3}\right)-\sqrt{ } \Sigma=0,
$$

the spherical pendulum relation.

\section{Landen's Transformation.}

15. It will be convenient at this stage to collect together all the formulas of Landen $\mathrm{s}$ transformation of the second order, required in the sequel, adopting the notation employed previously for the elliptic argument, $h K$ denoting an argument to modulus $k$ and $f K^{\prime}$ to modulus $k^{\prime}, h$ and $f$ denoting real fractions, as thereby the modulus is indicated in the notation and need not be written down.

Without this it will be difficult to reconcile the notation of different writers on this subject, each adopting a method of his own irrespective of others. 
Following Maxwell in his Electricity and Magnetism (E. and M.) §702, we select the LANDEN transformation connecting the new modulus $c$ and $c^{\prime}$ with $k$ and $k^{\prime}$ by the relation

$$
\begin{aligned}
& c^{\prime}=\frac{1-k}{1+k}, \quad c=\frac{2 \sqrt{ } k}{1+k}, \quad k=\frac{1-c^{\prime}}{1+c^{\prime}}, \\
& k^{\prime}=\frac{2 \sqrt{ } c^{\prime}}{1+c^{\prime}}, k^{\prime} c=2 \sqrt{ }\left(k c^{\prime}\right), \quad c>k, \quad c^{\prime}<k^{\prime},
\end{aligned}
$$

all different forms of the modular equation of the second order, connecting the modular angles $\gamma$ and $\beta$ shown in figures $A$ and $B$, such that

$$
\begin{gathered}
\cos \gamma=c^{\prime}=\frac{1-\sin \beta}{1+\sin \beta}=\tan ^{2}\left(\frac{1}{4} \pi-\frac{1}{2} \beta\right)=\frac{B C}{A C}, \\
k=\sin \beta=\frac{1-\cos \gamma}{1-\cos \gamma}=\tan ^{2} \frac{1}{2} \gamma=\frac{A C}{A} \frac{C}{C^{\prime}}=\frac{O C}{O A} .
\end{gathered}
$$

Then the complete elliptic integral or quarter-period $F\left(\frac{1}{2} \pi, c\right)$ or $F(c)$ is denoted by $F$, and $F\left(c^{\prime}\right)$ by $F^{\prime}, K$ and $K^{\prime}$ denoting $F(k)$ and $F^{\prime}\left(k^{\prime}\right)$; and

$$
\begin{array}{cc}
F=(1+k) K, & K=\frac{1}{2}\left(1+c^{\prime}\right) F, \\
F^{\prime}=\frac{1}{2}(1+k) K^{\prime}, & K^{\prime}=\left(1+c^{\prime}\right) F^{\prime}, \\
\frac{F^{\prime}}{F}=\frac{1}{2} \frac{K^{\prime}}{K}, & \frac{K^{\prime}}{K}=2 \frac{F^{\prime}}{F} .
\end{array}
$$

16. The geometrical interpretation of LANDEN's transformation is seen in fig. $B$, where we may put

so that

(3) $\quad \chi=\frac{1}{2} \pi+\omega-\omega^{\prime}, \quad$ am $2 h K=\frac{1}{2} \pi+\operatorname{am} h F-\operatorname{am}(1-h) F$,

$$
\begin{aligned}
\operatorname{sn} 2 h K & =\cos \left[\operatorname{am} h F-\operatorname{am}(1-h) F^{\prime}\right] \\
& =\operatorname{cn} h F \operatorname{cn}(1-h) F+\operatorname{sn} h F^{\prime} \operatorname{sn}(1-h) F=\left(1+c^{\prime}\right) \frac{\operatorname{sn} h F \operatorname{cn} h F}{\operatorname{dn} h K},
\end{aligned}
$$

LANDEN's first transformation; and putting $h=0$ leads to (3) $\S 15$.

Thence also

$$
\begin{aligned}
\operatorname{cn} 2 h K=\frac{1-\left(1+c^{\prime}\right) \operatorname{sn}^{2} h F}{\operatorname{dn} h F^{\prime}}=\frac{\operatorname{dn}^{2} h F-c^{\prime}}{\left(1-c^{\prime}\right) \operatorname{dn} h F} \\
=\frac{\operatorname{dn} h F-\operatorname{dn}(1-h) F}{1-c^{\prime}}=\frac{C Q-C Q^{\prime}}{C A-C B}=\cos \chi,
\end{aligned}
$$




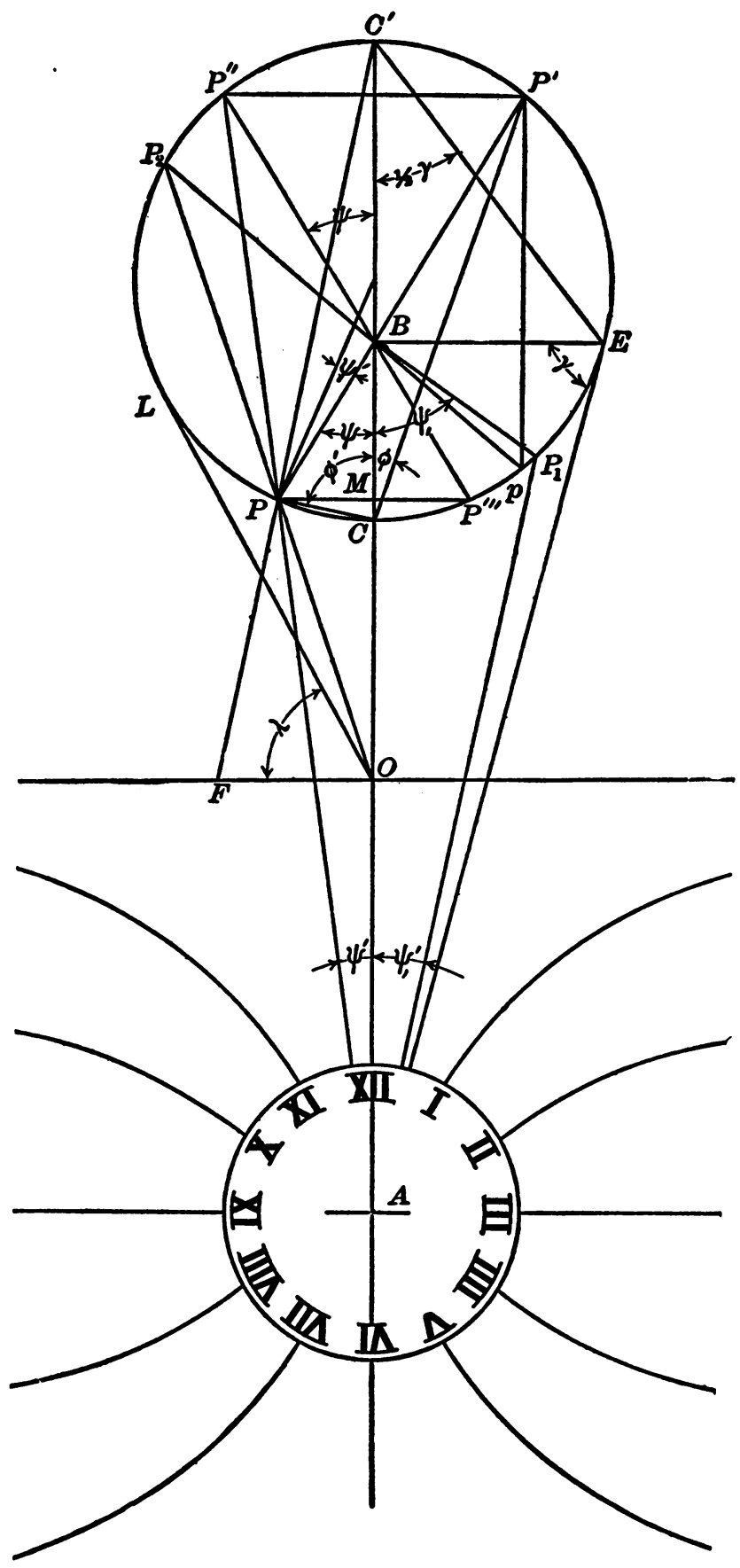

Fia. 4 . 
the geometrical interpretation, since $C Q, C Q^{\prime}, C B=C A \operatorname{dn}(f, 1-f, 1) F$;

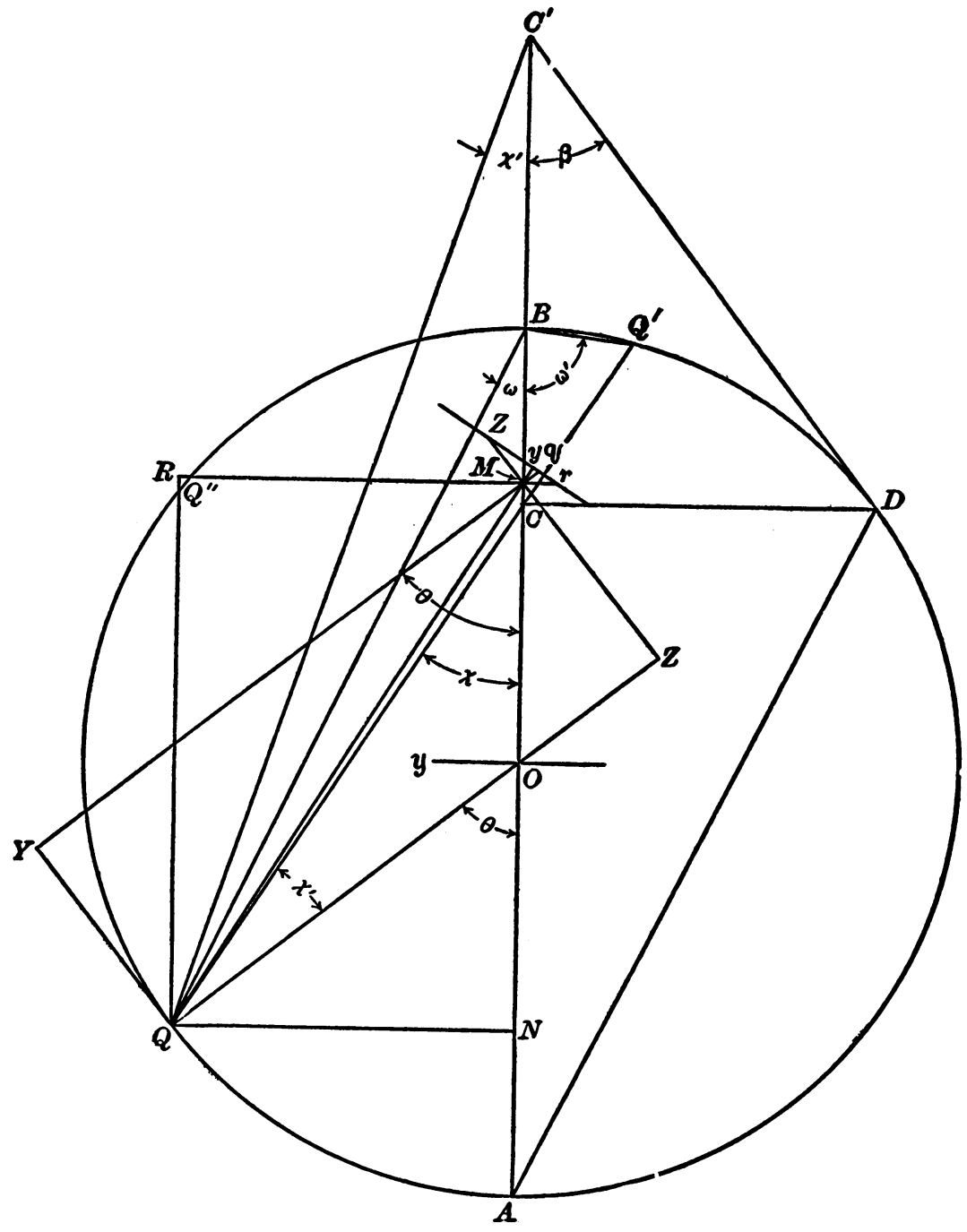

FIG. $B$.

(6) $\operatorname{dn} 2 h K=\frac{1-\left(1-c^{\prime}\right) \operatorname{sn}^{2} h F}{\operatorname{dn} h F^{\prime}}=\frac{\operatorname{dn}^{2} h F+c^{\prime}}{\left(1+c^{\prime}\right) \operatorname{dn} h F^{\prime}}=\frac{Q Q^{\prime}}{A B}$.

Equation (4) can be written

(7)

and thence

(8)

$$
\operatorname{sn}^{2} 2 h K=\frac{1+c^{\prime}}{1-c^{\prime}} \frac{1-\operatorname{dn} 2 h F}{1+\operatorname{dn} 2 h F^{\prime}},
$$

$$
\operatorname{dn} 2 h F=\frac{1-k \mathrm{sn}^{2} 2 h K}{1+k \mathrm{sn}^{2} 2 h K},
$$


which is LANDEN's II transformation, in conjunction with

$$
\begin{aligned}
& \text { sn } 2 h F=\frac{(1+k) \operatorname{sn} 2 h K}{1+k \operatorname{sn}^{2} 2 h K}, \\
& \text { en } 2 h F=\frac{\operatorname{cn} 2 h K \mathrm{dn} 2 h K}{1+k \operatorname{sn}^{2} 2 h K} .
\end{aligned}
$$

These may also be written, replacing $2 h$ by $h$,

(11) $\operatorname{sn} h F=\frac{1}{2} \sqrt{ }(1+\operatorname{sn} 2 h K \cdot 1+k \operatorname{sn} 2 h K)-\frac{1}{2} \sqrt{ }(1-\operatorname{sn} 2 h K \cdot 1-k \operatorname{sn} 2 h K)$,

(12) en $h F=\frac{1}{2} V(1+\operatorname{sn} 2 h K \cdot 1-k \operatorname{sn} 2 h K)+\frac{1}{2} V(1-\operatorname{sn} 2 h K \cdot 1+k \operatorname{sn} 2 h K)$,

$$
\begin{aligned}
\operatorname{dn} h F & =\frac{\operatorname{dn} 2 h K+k \operatorname{cn} 2 h K}{1+k}=\frac{1-k}{\operatorname{dn} 2 h K-k \operatorname{cn} 2 h K} \\
& =\sqrt{\frac{1-k}{1+k}} \sqrt{\frac{\operatorname{dn} 2 h K+k \operatorname{cn} 2 h K}{\operatorname{dn} 2 h K-k \operatorname{cn} 2 h K}} .
\end{aligned}
$$

17. The associated transformation of the complementary period $K^{\prime}$ or $F^{\prime}$ can now be written down and interpreted geometrically in fig. $A$ where

(1) $\psi=\operatorname{am} 2 f F^{\prime}, \phi=\operatorname{am} f K^{\prime}, \phi^{\prime}=\operatorname{am}(1-f) K^{\prime}, 2 \phi=\psi+\psi^{\prime}, \pi-2 \phi^{\prime}=\psi-\psi^{\prime}$, and as in LANDEN's II transformation in (11) $\S 16$, $\sin \phi=\sin \frac{1}{2}\left(\psi+\psi^{\prime}\right)=\frac{1}{2} V\left(1+\sin \psi \cdot 1+\sin \psi^{\prime}\right)-\frac{1}{2} V\left(1-\sin \psi 1-\sin \psi^{\prime}\right)$,

$$
\begin{aligned}
\operatorname{sn} f K^{\prime} & =\frac{1}{2} \sqrt{ }\left(1+\operatorname{sn} 2 f F^{\prime} \cdot 1+c^{\prime} \operatorname{sn} 2 f F^{\prime}\right)-\frac{1}{2} \sqrt{ }\left(1-\operatorname{sn} 2 f F^{\prime} \cdot 1-c^{\prime} \operatorname{sn} 2 f F^{\prime}\right) \\
& =\frac{\left(1+c^{\prime}\right) \operatorname{sn} f F^{\prime}}{1+c^{\prime} \operatorname{su}^{2} f F^{\prime}}
\end{aligned}
$$

as in (9) $\S 16$ so that $f=0$ leads to $(4) \S 15$;

$$
\text { cn } \begin{aligned}
f K^{\prime} & =\frac{1}{2} \sqrt{ }\left(1+\operatorname{sn} 2 f F^{\prime} \cdot 1-c^{\prime} \operatorname{sn} 2 f F^{\prime}\right)+\frac{1}{2} V\left(1-\operatorname{sn} 2 f F^{\prime \prime} 1+c^{\prime} \operatorname{sn} 2 f F^{\prime}\right) \\
& =\frac{\operatorname{cn} f F^{\prime \prime} \operatorname{dn} f F^{\prime}}{1+c^{\prime} \operatorname{sn}^{2} f F^{\prime \prime}},
\end{aligned}
$$

$$
\begin{aligned}
\operatorname{dn} f K^{\prime} & =\frac{1-c^{\prime} \operatorname{sn}^{2} f F^{\prime}}{1+c^{\prime} \operatorname{sn}^{2} f F^{\prime}}=\frac{\operatorname{dn} 2 f F^{\prime}+c^{\prime} \operatorname{cn} 2 f F^{\prime}}{1+c^{\prime}} \\
& =\frac{1-c^{\prime}}{\operatorname{dn} 2 f F^{\prime}-c^{\prime} \operatorname{cn} 2 f F^{\prime \prime}}=\sqrt{\frac{1-c^{\prime}}{1+c^{\prime}}} \sqrt{\frac{\operatorname{dn} 2 f F^{\prime}+c^{\prime} \operatorname{cn} 2 f F^{\prime}}{\operatorname{dn} 2 f F^{\prime \prime}-c^{\prime} \operatorname{cn} 2 f F^{\prime \prime}}}
\end{aligned}
$$

and, conversely,

$$
\operatorname{sn} 2 f F^{\prime}=(1+k) \frac{\operatorname{sn} f K^{\prime} \text { en } f K^{\prime}}{\operatorname{dn} f K^{\prime}}=\sqrt{\frac{1+k}{1-k}} \sqrt{\frac{1-\operatorname{dn} 2 f K^{\prime}}{1+\operatorname{dn} 2 f K^{\prime}},}
$$


derivable from $(2,3,4)$, and the geometrical interpretation of

(6) $\psi=\frac{1}{2} \pi+\phi-\phi^{\prime}, \quad \sin \psi=\cos \left(\phi-\phi^{\prime}\right), \quad \cos \psi=\sin \left(\phi^{\prime}-\phi\right)$;

$$
\begin{gathered}
\operatorname{cn} 2 f F^{\prime}=\frac{1-(1+k) \operatorname{sn}^{2} f K^{\prime}}{\operatorname{dn} f K^{\prime}}=\frac{\operatorname{dn}^{2} f K^{\prime}-k}{(1-k) \operatorname{dn} f K^{\prime}} \\
=\frac{\operatorname{dn} f K^{\prime}-\operatorname{dn}(1-f) K^{\prime}}{1-k}=\frac{B P^{\prime}-B P}{B C^{\prime}-B C}=\cos \psi, \\
\operatorname{dn} 2 f F^{\prime}=\frac{1-(1-k) \operatorname{sn}^{2} f K^{\prime}}{\operatorname{dn} f K^{\prime}}=\frac{\operatorname{dn}^{2} f K^{\prime}+k}{(1+k) \operatorname{dn} f K^{\prime}} \\
=\frac{\operatorname{dn} f K^{\prime}+\operatorname{dn}(1-f) K^{\prime}}{1+k}=\frac{B P^{\prime}+B P}{B C^{\prime}+B C}=\frac{P P^{\prime}}{C C^{\prime}}=\cos \psi^{\prime},
\end{gathered}
$$

the geometrical interpretation.

The two circles in figures $A$ and $B$, linked in perpendicular planes, may be taken to typify the magnetic and electric circuit, associated in a similar manner.

18. In LeGENDRE's notation, the II. E. I.

$$
E(\omega, c)=\int_{0}^{\omega} V\left(1-c^{2} \sin ^{2} \omega\right) d \omega=\int \Delta \omega d \omega,
$$

and the complete II. E. I., or $E\left(\frac{1}{2} \pi, c\right)$ or $E(c)$ is denoted by $E$, and $E(i)$ by $E^{\prime}$, while as before $E\left(\frac{1}{2} \pi, k\right)$ is denoted by $H$, and $E\left(k^{\prime}\right)$ by $H^{\prime}$.

In JACOBI's notation, with $\omega=\operatorname{am} h F$, and as employed already in $\S 3$,

$$
\int_{0}^{h} \operatorname{dn}^{2} h F \cdot F d h=E \operatorname{am} h F=h E+\operatorname{zn} h F .
$$

Squaring equation (13) $§ 16$,

$$
\mathrm{dn}^{2} h F=\frac{2 \mathrm{dn}^{2} 2 h K+2 k \operatorname{cn} 2 h K \mathrm{dn} 2 h K-k^{2}}{(1+k)^{2}},
$$

and then integrating with respect to $h$, there results

$$
\begin{aligned}
& \frac{h E+\mathrm{zn} h F}{F}=\frac{2 h H+\mathrm{zn} 2 h K+k \mathrm{sn} 2 h K-k^{\prime 2} h K}{(1+k)^{2} K}, \\
& h E+\operatorname{zn} h F=\frac{2 h H-k^{\prime 2} h K+\mathrm{zn} 2 h K+k \operatorname{sn} 2 h K}{1+k},
\end{aligned}
$$

so that, putting $h=1$,

$$
E=\frac{2 H-k^{\prime 2} K}{1+k}=\frac{2 H}{1+k}-(1-k) K,
$$




$$
F-E=2\left(K-\frac{H}{1+k}\right), \quad E-c^{\prime 2} F=2 \frac{H-(1-k) K}{1+k},
$$

and conversely

$$
H=\frac{E+c^{\prime} F}{1+c^{\prime}}, \quad H K^{\prime}=E F^{\prime}+c^{\prime} F F^{\prime},
$$

so that, as in Maxwell E. and M. $\S 701$ when corrected,

19. Then

$$
\begin{gathered}
\frac{2}{c}(F-E)-c F=2 \frac{K-H}{\sqrt{ }}, \\
2(F-E)-c^{2} F=4 \frac{K-H}{1+k}, \\
\left(1+c^{\prime 2}\right) E-2 c^{\prime 2} F=4 \frac{\left(1+k^{2}\right) H-\left(1-k^{2}\right) K}{(1+k)^{3}} .
\end{gathered}
$$

$$
\operatorname{zn} h F=\frac{\operatorname{zn} 2 h K+k \operatorname{sn} 2 h K}{1+k},
$$

which it is convenient to write

$$
F_{\mathrm{zn}} h F=K \mathrm{zn} 2 h K+K k \text { sn } 2 h K,
$$

and conversely

$$
\begin{aligned}
K \operatorname{mn} 2 h K & =F_{\mathrm{zn}} h F^{\prime}-\frac{1}{2} F^{2} \frac{\operatorname{sn} h F^{\prime} \mathrm{cn} h F}{\operatorname{dn} h F^{2}} \\
& =\frac{1}{2} F_{\mathrm{zn}} h F^{\prime}-\frac{1}{2} F^{\prime} \mathrm{zn}(1-h) F \\
& =\frac{1}{2} F^{\prime} \mathrm{zn} 2 h F^{\prime}-\frac{1}{2} F c^{2} \frac{\operatorname{sn}}{1} \frac{2 h F^{\prime} \operatorname{cn} h F}{\operatorname{dn} 2 h F^{\prime}} .
\end{aligned}
$$

Squaring (4) $§ 17$ and integrating with respect to $f$, we obtain the remaining relations

which we write

$$
\begin{gathered}
H^{\prime}=\frac{2 E^{\prime}}{1+c^{\prime}}-\left(1-c^{\prime}\right) F^{\prime}, \\
E^{\prime}=\frac{H^{\prime}+k K^{\prime}}{1+k}, \quad E^{\prime} F^{\prime}=H^{\prime} K+k K K^{\prime}, \\
\operatorname{zn} f K^{\prime}=\frac{\operatorname{zn} 2 f F^{\prime}+c^{\prime} \operatorname{sn} 2 f F^{\prime}}{1+c^{\prime}},
\end{gathered}
$$

$$
K \text { zn } f K^{\prime}=\quad \frac{1}{2} F \text { zn } 2 f F^{\prime}+\frac{1}{2} F c^{\prime} \text { sn } 2 f F^{\prime}
$$

and conversely

$$
F^{\prime} \operatorname{zn} 2 f F^{\prime}=2 K \operatorname{zn} f K^{\prime}-K k^{\prime 2} \frac{\operatorname{sn} f K^{\prime} \operatorname{cn} f K^{\prime}}{\operatorname{dn} f K^{\prime}}
$$




$$
\begin{gathered}
=K \operatorname{zn} f K^{\prime}-K \operatorname{mn}(1-f) K^{\prime} \\
=K \operatorname{zn} 2 f K^{\prime}-K k^{\prime 2} \frac{\operatorname{sn} 2 f K^{\prime} \operatorname{cn} 2 f K^{\prime}}{1+\operatorname{dn} 2 f K^{\prime}} \\
=K \operatorname{zs} 2 f K^{\prime}-K \frac{\operatorname{cn} 2 f F^{\prime}}{\operatorname{sn} 2 f K^{\prime}}, \\
F^{\prime} 2 \mathrm{n}(1-2 f) F^{\prime}=K \operatorname{zs}(1-2 f) K^{\prime}-K k \operatorname{tn} 2 f K^{\prime},
\end{gathered}
$$

theorems required in the sequel for the quadric transformation of $M$, the mutual induction of a helix and a coaxial ring.

20. Integrating (3) $\S 19$ with respect to $h$,

$$
\begin{gathered}
\log \frac{\Theta 2 h K}{\Theta 0 K}=2 \log \frac{\Theta h F}{\Theta 0 F}+\log \operatorname{dn} h F, \\
\frac{\Theta 2 h K}{\Theta 0 K}=\left(\frac{\Theta h F}{\Theta 0 F}\right)^{2} \operatorname{dn} h F=\frac{\Theta h F}{\Theta 0 F} \frac{\Theta(1-h) F}{\Theta F},
\end{gathered}
$$

the quadric transformation of the theta function.

Similarly by integration of $(9), \S 19$, with respect to $f$,

$$
\frac{\Theta 2 f F^{\prime}}{\Theta 0 F^{\prime}}=\left(\frac{\Theta f K^{\prime}}{\Theta 0 K^{\prime}}\right)^{2} \operatorname{dn} f K^{\prime}=\frac{\Theta f K^{\prime}}{\Theta 0 K^{\prime}} \frac{\Theta(1-f) K^{\prime}}{\Theta K^{\prime}} .
$$

The same notation is useful for expressing the change in a theta function and eta function from imaginary to real argument and comodulus in the form

$$
\frac{\Theta\left(2 f K^{\prime} i, k\right)}{\Theta(0, k)}=q^{-f^{2}} \frac{H(1-2 f) K^{\prime}}{H K^{\prime}}, \quad \frac{H\left(2 f K^{\prime} i, k\right)}{H(K, k)}=i q^{-f^{2}} \frac{H 2 f K^{\prime}}{H K^{\prime}},
$$

where $q=\exp \left(-\pi K^{\prime} / K\right)$. (J ACOBI, Fundamenta nova, or Werke, I, p. 215; Ca YLeY, Elliptic Functions, p. 151.)

These transformations may be used for the bisection of the elliptic function;

$$
\begin{aligned}
\left(\frac{\Theta h F}{\Theta 0 F}\right)^{2} & =\frac{\Theta 2 h K}{\Theta 0 K} \frac{\mathrm{dn} 2 h K-k \mathrm{cn} 2 h K}{1-k} \\
& =\frac{1}{1-k} \stackrel{\Theta(1-2 h) K}{\Theta 0 K}-\frac{k}{1-k} \frac{H(1-2 h) K}{H K}, \\
\left(\frac{\Theta h F}{\Theta F}\right)^{2} & =\frac{1}{1+k} \frac{\Theta(1-2 h) K}{\Theta 0 K}-\frac{k}{1+k} \frac{H(1-2 h) K}{H K} .
\end{aligned}
$$


Algebraical form of Landen's transformations.

21. The algebraical equivalent of our first quadric transformation in $\S 16$ is (TANNERY and MolK, Fonctions elliptiques, formulas XXII, XXIV)

$$
\begin{aligned}
& m^{2}\left(s-s_{1}\right)=\frac{\left[t-t_{1}-V\left(t_{1}-t_{2} \cdot t_{1}-t_{3}\right)\right]^{2}}{4\left(t-t_{1}\right)}, \\
& m^{2}\left(s-s_{2}\right)=\frac{\left[t-t_{1}+V\left(t_{1}-t_{3} \cdot t_{2}-t_{3}\right)\right]^{2}}{4\left(t-t_{1}\right.}, \\
& m^{2}\left(s-s_{3}\right)=\frac{t-t_{2} t-t_{3}}{4\left(t-t_{1}\right)}=\frac{T}{16\left(t-t_{1}\right)^{2}},
\end{aligned}
$$

so that the graph of a relation $(s, t)$ is a hyperbola; and

$$
\begin{aligned}
& 2 m \vee\left(s_{1}-s_{3}\right)=V\left(t_{1}-t_{3}\right)-V\left(t_{1}-t_{2}\right), \\
& 2 m V\left(s_{2}-s_{3}\right)=V\left(t_{1}-t_{3}\right)-V\left(t_{1}-t_{2}\right),
\end{aligned}
$$

the equivalent of the modular equation $(1) \S 15$, with $k, k^{\prime}$ in terms of $s_{1}, s_{2}, s_{3}$, as in (4) $\S 2$, and $c, c^{\prime}$ the equivalent for $t_{1}, t_{2}, t_{3}$. Also

where

$$
\begin{gathered}
m^{2} \frac{d s}{d t}=\frac{\left(t-t_{1}\right)^{2}-\left(t_{1}-t_{2} \cdot t_{1}-t_{3}\right)}{4\left(t-t_{1}\right)^{2}}, \\
m^{3} \vee S=\frac{\left(t-t_{1}\right)^{2}-\left(t_{1}-t_{2} \cdot t_{1}-t_{3}\right)}{8\left(t-t_{1}\right)^{2}} \vee T,
\end{gathered}
$$

$$
\begin{gathered}
S=4\left(s-s_{1} \cdot s-s_{2} \cdot s-s_{3}\right), \quad T=4\left(t-t_{1} \cdot t-t_{2} \cdot t-t_{3}\right), \\
\frac{d s}{m \sqrt{ } S}=2 \frac{d t}{\sqrt{ } T} .
\end{gathered}
$$

This algebraical result is obtained by substitution in (4), (5), (6), $\S 15$, of

(11) $\sin ^{2} \omega=\operatorname{sn}^{2} h F=\frac{t_{1}-t_{3}}{t-t_{3}}$, or $\frac{t-t_{3}}{t_{2}-t_{3}}, \infty>t>t_{1}$, or $t_{2}>t>t_{3}$.

In the associated complementary transformation of $\S 18$, writing $\sigma$ and $\Sigma$ for $s$ and $S, \tau$ and $T^{\prime}$ for $t$ and $T$, the region $s_{1}>\sigma>s_{2}$ is excluded, because $\sigma-s_{1}$ and $\sigma-s_{2}$ must have the same sign; but

$$
\sin ^{2} \phi=\operatorname{sn}^{2} f K^{\prime}=\frac{s_{1}-s_{3}}{s_{1}-\sigma}, \quad s_{3}>\sigma>-\infty .
$$




$$
\operatorname{sn}^{2} f F^{\prime}=\frac{t_{1}-\tau}{t_{1}-t_{2}}, \text { or } \frac{t_{1}-t_{3}}{t_{1}-\tau}, t_{1}>\tau>t_{2}, \text { or } t_{3}>\tau>-\infty,
$$

and these substituted in (2), (3), §17, will satisfy the algebraical relation above.

On applying this algebraical quadric transformation to our III. E. I.,

$$
\begin{gathered}
m^{3} V(-\Sigma)=\frac{\left(\tau-t_{1}\right)^{2}-\left(t_{1}-t_{2} \cdot t_{1}-t_{3}\right)}{8\left(\tau-t_{1}\right)^{2}} V\left(-T^{\prime}\right) \\
m^{2}(s-\sigma)=\frac{(t-\tau)\left[\left(t-t_{1}\right)\left(\tau-t_{1}\right)-\left(t_{1}-t_{2} \cdot t_{1}-t_{3}\right)\right]}{4\left(t-t_{1}\right)\left(\tau-t_{1}\right)} \\
\frac{2 m V(-\Sigma)}{s-\sigma}=\frac{V\left(-T^{\prime}\right)}{t-\tau}-\frac{\left(t_{1}-t_{2} \cdot t_{1}-t_{3}\right) V\left(-T^{\prime}\right)}{\left(\tau-t_{1}\right)\left[\left(t-t_{1} \cdot \tau-t_{1}\right)-\left(t_{1}-t_{2} \cdot t_{1}-t_{3}\right)\right]} \\
=\frac{V\left(-T^{\prime}\right)}{t-\tau}+\frac{V\left(-T^{\prime}\right)}{u-\tau}+\frac{V\left(-T^{\prime}\right)}{\tau-t_{1}}
\end{gathered}
$$

and on putting

so that

$$
\frac{\sqrt{ }(-\Sigma)}{s-\sigma} \frac{d s}{\sqrt{ } S}=\frac{V\left(-T^{\prime}\right)}{t-\tau} \frac{d t}{\sqrt{ } T}-\frac{V\left(-T^{\prime}\right)}{u-\tau} \frac{d u}{\sqrt{ } U}-\frac{\sqrt{ }\left(-T^{\prime}\right)}{t_{1}-\tau} \frac{d t}{\sqrt{ } T} .
$$

The hyperbolic graph connecting $s$ and $t$ being drawn it shows that as $t$ increases from $t_{1}$ to $\infty, u$ diminishes from $\infty$ to $t_{1}$ and $s$ diminishes from $\infty$ to $s_{1}$ and rises again to infinity so that

$$
\int_{s_{1}}^{\infty} \frac{\sqrt{ }(-\Sigma)}{s-\sigma} \frac{d s}{\sqrt{ } S}=-\int_{t_{1}}^{\infty} \frac{\sqrt{ }\left(-T^{\prime}\right)}{t-\tau} \frac{d t}{\sqrt{ } T}+\sqrt{\left(\frac{\tau-t_{1} \cdot \tau-t_{3}}{t_{1}-\tau \cdot t_{1}-t_{3}}\right) \int \frac{V\left(t_{1}-t_{3}\right) d t}{V T}}
$$

(21) $2 C\left(f K^{\prime}\right)=-2 A\left(f F^{\prime}\right)+\frac{\operatorname{cn} f F^{\prime} \operatorname{dn} f F^{\prime}}{\operatorname{sn} f F^{\prime}} F=-A\left(f F^{\prime}\right)+C\left(f F^{\prime \prime}\right)$.

But as $t$ increases from $t_{3}$ to $t_{2}, u$ diminishes from $t_{2}$ to $t_{3}$, and $s$ increases from $s_{3}$ to $s_{2}$ and diminishes again to $s_{3}$, so that

$$
\int_{s_{3}}^{s_{2}} \frac{V(-\Sigma)}{s-\sigma} \frac{d s}{\sqrt{ } S}=\int_{t_{3}}^{t_{2}} \frac{\sqrt{ }\left(-T^{\prime}\right)}{t-\tau} \frac{d t}{\sqrt{ } T}+\sqrt{ }\left(\frac{\tau-t_{2} \cdot \tau-t_{3}}{t_{1}-\tau \cdot t_{1}-t_{3}}\right) \int \frac{\sqrt{ }\left(t_{1}-t_{3}\right) d t}{\sqrt{ } T}
$$

(23) $2 D\left(f K^{\prime}\right)=2 B\left(f F^{\prime}\right)+\frac{\operatorname{cn} f F^{\prime} \operatorname{dn} f F^{\prime}}{\operatorname{sn} f F^{\prime}} F=B\left(f F^{\prime}\right)+D\left(f F^{\prime}\right)$. 
Making use of the expressions for $A, B, C, D$ in $\S 5, \S 6$, equations (21), (23) are equivalent to the single relation

$$
2 K_{\mathrm{zs}} f K^{\prime}=F_{\mathrm{zs}} f F^{\prime}+F \mathrm{zn} f F^{\prime},
$$

as before in $\S 19$.

22. For the transformation from $t$ back to $s$ in the III. E. I., we take

$$
\begin{aligned}
& \frac{t-t_{1}}{t_{1}-\tau}=\frac{V\left(s-s_{2}\right)+V\left(s-s_{1}\right)}{V\left(s-s_{2}\right)-V\left(s-s_{1}\right)} \quad \frac{V\left(s_{1}-\sigma\right)-V\left(s_{2}-\sigma\right)}{\sqrt{ }\left(s_{1}-\sigma\right)+V\left(s_{2}-\sigma\right)}, \\
& \frac{t-\tau}{t_{1}-\tau}=2 \frac{V\left(s-s_{2}\right) V\left(s_{1}-\sigma\right)-V\left(s-s_{1}\right) V\left(s_{2}-\sigma\right)}{\left[V\left(s-s_{2}\right)-V\left(s-s_{1}\right)\right]\left[V\left(s_{1}-\sigma\right)+V\left(s_{2}-\sigma\right)\right]}, \\
& {\left[V\left(s_{1}-\sigma\right)+V\left(s_{2}-\sigma\right)\right]\left[V\left(s_{1}-s_{2}\right) V\left(s-s_{1}\right)\right]} \\
& \frac{t_{1}-\tau}{t-\tau}=\frac{\left.\times\left[s-s_{2}\right) V(s-\sigma)+V\left(s-s_{1}\right) V\left(s_{2}-\sigma\right)\right]}{2\left(s_{1}-s_{2}\right)(s-\sigma)} \\
& =\frac{1}{2}+\frac{V\left(s_{1}-\sigma \cdot s_{2}-\sigma\right)-V\left(s-s_{1} \cdot s-s_{2}\right)}{2(s-\sigma)}, \\
& \frac{\frac{1}{2} V\left(-T^{\prime}\right)}{t_{1}-\tau}=2 \vee\left(s_{3}-\sigma\right) \text {, } \\
& \frac{\frac{1}{2} V\left(-T^{\prime}\right)}{t-\tau} \frac{d t}{\sqrt{ } T}=\left[\frac{1}{2}+\frac{\sqrt{ }\left(s_{1}-\sigma \cdot s_{2}-\sigma\right)-V\left(s-s_{1} \cdot s-s_{2}\right)}{2(s-\sigma)}\right] \frac{\sqrt{ }\left(s_{3}-\sigma\right) d s}{\sqrt{ } S} \\
& \int_{t_{1}, t_{3}}^{\infty, t_{2}} \frac{\frac{1}{2} V\left(-T^{\prime}\right)}{t-\tau} \frac{d t}{\sqrt{T}}
\end{aligned}
$$

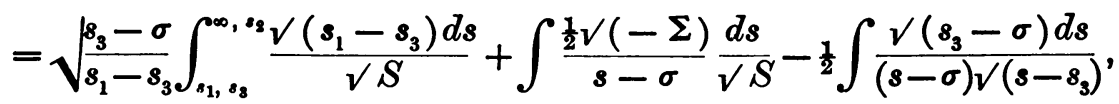

the last integral being zero as $s$ ranges forward and back again.

According to the region of $\tau$,

$$
t_{1}>\tau>t_{2}, \quad \text { or } \quad t_{2}>\tau>-\infty,
$$

and with

the relation (6) is equivalent to

$$
s_{3}>\sigma>-\infty
$$

$$
\begin{aligned}
A\left(f F^{\prime}\right) & =K \frac{\mathrm{cn} f K^{\prime}}{\operatorname{sn} f K^{\prime}}-C\left(f K^{\prime}\right), \\
-B\left(f F^{\prime}\right) & =K \frac{\mathrm{cn} f K^{\prime}}{\operatorname{sn} f K^{\prime}}-D\left(f K^{\prime}\right), \\
C\left(f F^{\prime}\right) & =K \frac{\mathrm{cn} f K^{\prime}}{\operatorname{sn} f K^{\prime}}+C\left(f K^{\prime}\right),
\end{aligned}
$$




$$
D\left(f F^{\prime}\right)=K \frac{\mathrm{cn} f K^{\prime}}{\operatorname{sn} f K^{\prime}}+D\left(f K^{\prime}\right),
$$

and these reduce to the single relation

$$
F_{\mathrm{zn}} f F^{\prime}=K \mathrm{zs} f K^{\prime}-K \frac{\mathrm{cn} f K^{\prime}}{\operatorname{sn} f K^{\prime}}
$$

23. The algebraical equivalent of the second quadric transformation in (8), (9), (10), § 16, is in a similar way equivalent to a hyperbolic graph between $x$ and $s$,

$$
\begin{aligned}
& M^{2}\left(x-t_{1}\right)=\frac{8-s_{1} \cdot 8-s_{2}}{8-s_{3}}=\frac{1 S}{\left(8-s_{3}\right)^{2}}, \\
& M^{2}\left(x-t_{2}\right)=\frac{\left[8-s_{3}-V\left(8_{1}-s_{3} \cdot s_{2}-s_{3}\right)\right]}{8-s_{3}}, \\
& M^{2}\left(x-t_{3}\right)=\frac{\left[8-s_{3}+V\left(s_{1}-s_{3} \cdot s_{2}-s_{3}\right)\right]^{2}}{8-s_{3}},
\end{aligned}
$$

and as in the modular equation (1) $\S 15$,

$$
\begin{aligned}
& M V\left(t_{1}-t_{3}\right)=V\left(s_{1}-s_{3}\right)+V\left(s_{2}-s_{3}\right), \\
& M V\left(t_{1}-t_{2}\right)=V\left(s_{1}-s_{3}\right)-V\left(s_{2}-s_{3}\right) .
\end{aligned}
$$

Also $M m=1$, and

$$
\begin{gathered}
M^{2} \frac{d x}{d s}=\frac{\left(s-s_{3}\right)^{2}-\left(s_{1}-s_{3} \cdot s_{2}-s_{3}\right)}{\left(8-s_{3}\right)^{2}}, \\
M^{3} \vee X=\frac{\left(8-s_{3}\right)^{2}-\left(s_{1}-s_{3} \cdot s_{2}-s_{3}\right)}{\left(8-s_{3}\right)^{2}} \vee S, \\
\frac{d x}{M V X}=\frac{d s}{\sqrt{ } S}, \quad X=4\left(x-t_{1} \cdot x-t_{2} \cdot x-t_{3}\right) .
\end{gathered}
$$

The region $t_{2}>x>t_{3}$ is now excluded, because $x-t_{2}$ and $x-t_{3}$ must have the same sign; but

$$
\sin ^{2} \xi=\operatorname{sn}^{2} 2 h F=\frac{t_{1}-t_{3}}{x-t_{3}}, \quad \infty>x>t_{1},
$$

$$
\sin ^{2} \chi=\operatorname{su}^{2} 2 h K=\frac{8_{1}-s_{3}}{8-s_{3}} \text { or } \frac{8-s_{3}}{s_{2}-s_{3}}, \quad \infty>8>8_{1} \text { or } 8_{2}>8>8_{3}
$$

and these substituted in (8), (9), (10) $§ 16$ will give the algebraical result above. 
In the associated complementary transformation with $\sigma, \Sigma$ for $s, S$ and $x^{\prime}, X^{\prime}$ for $x, X$,

$$
\sin ^{2} \phi=\operatorname{sn}^{2} f K^{\prime}=\frac{s_{1}-\sigma}{s_{1}-s_{2}} \text { or } \frac{s_{1}-s_{3}}{s_{1}-\sigma}, s_{1}>\sigma>s_{2}, \text { or } s_{3}>\sigma>-\infty,
$$

$$
\sin ^{2} \psi=\operatorname{sn}^{2} 2 f K^{\prime}=\frac{t_{1}-x^{\prime}}{t_{1}-t_{2}} \text { or } \frac{t_{1}-t_{3}}{t_{1}-x^{\prime}}, t_{1}>x^{\prime}>t_{2}, \text { or } t_{3}>x^{\prime}>-\infty,
$$

and the algebraical relation is satisfied when these are substituted in (5) $\$ 17$.

In the transformation of the III. E. I.,

$$
\begin{gathered}
M^{2}\left(x-x^{\prime}\right)=\frac{(s-\sigma)\left[\left(s-s_{3} \cdot \sigma-s_{3}\right)-\left(s_{1}-s_{3} \cdot s_{2}-s_{3}\right)\right]}{\left(s-s_{3}\right)\left(\sigma-s_{3}\right)} \\
M^{3} \vee\left(-X^{\prime}\right)=\frac{\left(\sigma-s_{3}\right)^{2}-\left(s_{1}-s_{3} \cdot s_{2}-s_{3}\right)}{\left(\sigma-s_{3}\right)^{2}} V(-\Sigma) \\
\frac{M V\left(-X^{\prime}\right)}{x-x^{\prime}}=\frac{\left(s-s_{3}\right)\left[\left(\sigma-s_{3}\right)^{2}-\left(s_{1}-s_{3} \cdot s_{2}-s_{3}\right)\right] V\left(-\Sigma^{\prime}\right)}{\left(\sigma-s_{3}\right)\left[\left(s-s_{3} \cdot \sigma-s_{3}\right)-\left(s_{1}-s_{3} \cdot s_{2}-s_{3}\right)\right](s-\sigma)} \\
=\frac{V(-\Sigma)}{s-\sigma}-\frac{V\left(-\Sigma^{\prime \prime}\right)}{s-\sigma^{\prime \prime}}
\end{gathered}
$$

where

$$
\sigma^{\prime \prime}-s_{3}=\frac{s_{1}-s_{3} s_{2}-s_{3}}{\sigma-s_{3}}, \quad \sigma^{\prime \prime}-s_{2}=\frac{s_{2}-s_{3} \cdot s_{1}-\sigma}{\sigma-s_{3}}, \quad \sigma^{\prime \prime}-s_{1}=\frac{s_{1}-s_{3} \cdot s_{2}-\sigma}{\sigma-s_{3}} .
$$

In the arrangement

and in the arrangement

$$
\begin{gathered}
s_{1}>\sigma>s_{2}, \quad s_{1}>\sigma^{\prime \prime}>s_{2}, \\
\mathrm{sn}^{2} f^{\prime \prime} K^{\prime}=\frac{s_{1}-\sigma^{\prime \prime}}{s_{1}-s_{2}}=\frac{s_{1}-s_{3} \sigma-s_{2}}{s_{1}-s_{2}} \frac{\mathrm{cn}^{2} f K^{\prime}}{\mathrm{dn}^{2} f K^{\prime}},
\end{gathered}
$$

so that

$$
\begin{gathered}
s_{3}>\sigma>-\infty, \quad s_{3}>\sigma^{\prime \prime}>-\infty, \\
\operatorname{sn}^{2} f^{\prime \prime} K^{\prime}=\frac{s_{1}-s_{3}}{s_{1}-\sigma^{\prime \prime}}=\frac{s_{3}-\sigma}{s_{2}-\sigma}=\frac{\mathrm{cn}^{2} f K^{\prime}}{\mathrm{dn}^{2} f K^{\prime}},
\end{gathered}
$$

The integral relation

$$
\int \frac{\frac{1}{2} \sqrt{ }\left(-X^{\prime}\right)}{x-x^{\prime}} \frac{d x}{\sqrt{ } X}=\int \frac{\frac{1}{2} \sqrt{ }(-\Sigma)}{s-\sigma} \frac{d s}{\sqrt{ } S}-\int \frac{\frac{1}{2} \sqrt{ }\left(-\Sigma^{\prime \prime}\right)}{s-\sigma^{\prime \prime}} \frac{d s}{\sqrt{ } S},
$$

is now equivalent to

(23) $A\left(2 f F^{\prime}\right)=A\left(f K^{\prime}\right)-A(1-f) K^{\prime}$, or $=-B\left(f K^{\prime}\right)+B(1-f) K^{\prime}$, 
implying the relation

$$
F_{\mathrm{zn}} 2 f F^{\prime}=K \mathrm{zn} f K^{\prime}-K \mathrm{zn}(1-f) K^{\prime},
$$

as in (9) $\S 19$; or

$$
C\left(2 f F^{\prime}\right)=C\left(f K^{\prime}\right)-C(1-f) K^{\prime}, \text { or }=D\left(f K^{\prime}\right)-D(1-f) K^{\prime},
$$

implying the equivalent to (24), when (5), (7), (8), § 17 are employed :

$$
F \mathrm{zs} 2 f F^{\prime}=K \mathrm{zs} f K^{\prime}-K \mathrm{zs}(1-f) K^{\prime} .
$$

Thus in transformation $I, h F$ and $f F^{\prime}$ become changed to $2 h K$ and $f K^{\prime}$, and in transformation II, $2 h K$ and $f K^{\prime}$ are changed into $2 h F$ and $2 f F^{\prime}$, a successive application being equivalent to a duplication of $h F$ and $f F^{\prime}$.

24. To proceed from $s$ to $x$ by means of the II transformation, take

$$
\begin{aligned}
& \frac{s-s_{3}}{\sigma-s_{3}}=\frac{V\left(x-t_{3}\right)+V\left(x-t_{2}\right)}{\sqrt{ }\left(x-t_{3}\right)-V\left(x-t_{2}\right)} \cdot \frac{V}{V\left(x^{\prime}-t_{3}\right)-V\left(x^{\prime}-t_{2}\right)}, \\
& \frac{s-\sigma}{\sigma-s_{3}}=2 \frac{V\left(x-t_{2}: x^{\prime}-t_{3}\right)-V\left(x-t_{3} \cdot x^{\prime}-t_{2}\right)}{\left[\sqrt{ }\left(x-t_{3}\right)-V\left(x-t_{2}\right)\right]\left[V\left(x^{\prime}-t_{3}\right)+V\left(x^{\prime}-t_{2}\right)\right]}, \\
& \frac{\sigma-s_{3}}{s-\sigma}=\frac{V\left(x^{\prime}-t_{2} \cdot x^{\prime}-t_{3}\right)+\sqrt{ }\left(x-t_{2} \cdot x-t_{3}\right)}{2\left(x-x^{\prime}\right)}-\frac{1}{2}, \\
& \frac{\frac{1}{2} V(-\Sigma)}{\sigma-s_{3}}=N \sqrt{ }\left(t_{1}-x^{\prime}\right),
\end{aligned}
$$

$$
\begin{gathered}
\frac{V(-\Sigma)}{s-\sigma} \frac{d s}{\sqrt{ } S}=\left[\frac{V\left(x^{\prime}-t_{2} \cdot x^{\prime}-t_{3}\right)+V\left(x-t_{2} \cdot x-t_{3}\right)}{x-x^{\prime}}-1\right] \frac{V\left(t_{1}-x^{\prime}\right) d x}{\sqrt{X}} \\
\int_{s_{1}, s_{2}}^{\infty, s_{3}} \frac{V(-\Sigma)}{s-\sigma} \frac{d s}{\sqrt{ } S}=\int_{t_{1}}^{\infty} \frac{\frac{1}{2} V\left(-X^{\prime}\right)}{x-x^{\prime}} \frac{d x}{\sqrt{ } X} \\
\quad+\frac{1}{2} \int_{t_{1}}^{\infty} \frac{V\left(t_{1}-x^{\prime}\right) d x}{\left(x-x^{\prime}\right) \sqrt{ }\left(x-t_{1}\right)}-\sqrt{\frac{t_{1}-x^{\prime}}{t_{1}-t_{3}}} \int_{t_{1}}^{\infty} \frac{V\left(t_{1}-t_{3}\right) d x}{\sqrt{ } X^{2}}
\end{gathered}
$$

of which the second integral is $\frac{1}{2} \pi$, and the third is $F c^{\prime}$ sn $2 f F^{\prime}$ or $F$ sn $2 f F^{\prime}$, according as

$$
t_{1}>x>t_{2} \text { or } t_{3}>x>-\infty ;
$$

so that in the region $s_{1}>\sigma>s_{2}, t_{1}>x>t_{2}$,

equivalent to

$$
\begin{aligned}
& 2 A\left(f K^{\prime}\right)=A\left(2 f F^{\prime}\right)+\frac{1}{2} \pi-F c^{\prime} \text { sn } 2 f F^{\prime}, \\
& 2 B\left(f K^{\prime}\right)=A\left(2 f F^{\prime}\right)-\frac{1}{2} \pi-F c^{\prime} \text { sn } 2 f F^{\prime},
\end{aligned}
$$

$$
2 K \mathrm{zn} f K^{\prime}=F \mathrm{zn} 2 f F^{\prime}+F c^{\prime} \operatorname{sn} 2 f F^{\prime},
$$


as in (7) $\S 19$; and in the region $s_{3}>\sigma>-\infty, t_{3}>x^{\prime}>-\infty$,

$$
\begin{aligned}
& 2 C\left(f K^{\prime}\right)=C\left(2 f F^{\prime}\right)-\frac{1}{2} \pi-\frac{F}{\operatorname{sn} 2 f F^{\prime \prime}}, \\
& 2 D\left(f K^{\prime}\right)=C\left(2 f F^{\prime \prime}\right)+\frac{1}{2} \pi-\frac{F}{\operatorname{sn} 2 f F^{\prime \prime}},
\end{aligned}
$$

equivalent, as in (7) $\S 19$, to

$$
2 K \mathrm{zs} f K^{\prime}=F \mathrm{zs} 2 f F^{\prime}-F \mathrm{~ns} 2 f F^{\prime} .
$$

25. Another form of LANDEN's transformation may be added here; put

$$
\begin{aligned}
& k=\operatorname{th} \alpha, k^{\prime}=\operatorname{sech} \alpha, \text { then } c^{\prime}=e^{-2 \alpha,} \\
& c^{\prime}=\operatorname{th} \delta, c=\operatorname{sech} \delta, \text { then } k=e^{-2 \delta},
\end{aligned}
$$

and then, as a form of the modular equation of the second order,

$$
\left(e^{2 \alpha}-1\right)\left(e^{2 \delta}-1\right)=2, \quad \operatorname{sh} 2 \alpha \operatorname{sh} 2 \delta=1 .
$$

Now with $\omega=\operatorname{am} h F$, put $\Delta \omega=$ th $\zeta$,

$$
\sin \omega=\frac{\operatorname{ch} \delta}{\operatorname{ch} \zeta}, \quad \cos \omega=\frac{V\left(\operatorname{ch}^{2} \zeta-\operatorname{ch}^{2} \delta\right)}{\operatorname{ch} \zeta}, \quad \operatorname{cn}(1-h) F=\frac{\operatorname{sh} \delta}{\operatorname{sh} \zeta},
$$

$$
F d h=\frac{d \omega}{\Delta \omega}=-\frac{\operatorname{ch} \delta d \zeta}{\left.\sqrt{\left(\operatorname{ch}^{2}\right.} \zeta-\operatorname{ch}^{2} \delta\right)},
$$

and from (7) $\S 16$,

$$
k \operatorname{sn}^{2} h K=e^{-2 \zeta}, \quad \text { sn } h K=e^{-\zeta+\delta} .
$$

In the plane $x O z$ of Fig. $A, v$ and $w$ of $\S 24$ are elliptic coördinates, to employ on WeIR's azimuth diagram; while $\phi$ and $k$ or $\alpha$ are dipolar coördinates, suitable for a stereographic projection or chart, with poles at $A$ and $B$, in which $2 \phi$ is the longitude of $P, 2 \phi^{\prime}$ of $P^{\prime}$ both on latitude $\lambda$, where

(6) $\sin \lambda=$ th $2 \alpha, \quad \cos \lambda=\operatorname{sech} 2 \alpha, \quad \tan \lambda=\operatorname{sh} 2 \alpha, \quad \tan \frac{1}{2} \lambda=$ th $\alpha=k$.

We can put, for the conformal representation of the stereographic projection,

$$
z+x i=a \tan (\phi+\alpha i),
$$

$$
\frac{x}{a}=\frac{\operatorname{sh} 2 \alpha}{\operatorname{ch} 2 \alpha+\cos 2 \phi}, \quad \frac{z}{a}=\frac{\sin 2 \phi}{\operatorname{ch} 2 \alpha+\cos 2 \phi},
$$

$$
\frac{P A, P B}{a}=\frac{r_{1}, r_{2}}{a}=\frac{e^{ \pm a}}{\sqrt{ }\left[\frac{1}{2}(\operatorname{ch} 2 \alpha+\cos 2 \phi)\right]},
$$

and if $O P P_{2}$ is a straight line

$$
\phi+\phi_{2}=\frac{1}{2} \pi \text {. }
$$


But if $A P P^{\prime \prime}$ is a straight line

$$
\psi+\psi^{\prime \prime}=\pi
$$

and $\psi, \psi^{\prime}$ are biangular coördinates of $P$, so that $\psi^{\prime \prime}=\operatorname{am} 2(1-f) F^{\prime}$, with the value of $\psi, \phi, \phi^{\prime}$ in $(1) \S 17$; also

$$
\begin{gathered}
B P=\frac{a(1-k)}{\operatorname{dn} f K^{\prime}}, \quad A P=\frac{a(1+k)}{\operatorname{dn} f K^{\prime}}, \\
B P^{\prime}=B P^{\prime \prime}=\frac{a(1-k)}{\operatorname{dn}(1-f) K^{\prime}}, \quad A P^{\prime}=A P^{\prime \prime}=\frac{a(1+k)}{\operatorname{dn}(1-f) K^{\prime}}, \\
O C \cdot O C^{\prime}=a^{2}=O B^{2}, \quad P B \cdot B P^{\prime}=O B^{2} \frac{(1-k)^{2}}{k}, \\
O C^{\prime}=\frac{a}{k}, \quad O C=a k, \quad C^{\prime} B=a\left(\frac{1}{k}-1\right), \quad B C=a(1-k) .
\end{gathered}
$$

Coefficient of mutual induction of two coaxial circles.

26. Apply the preceding method to Maxwell's $\S 701$ (E. and M.). The mutual induction of a short element of wire at $P$ perpendicular to the plane $A P B$ of fig. $A$ and of a sircular wire on a diameter $A B$ in the perpendicular plane of fig. $B$, is, for the arc $A Q$ where $A O Q=\theta$,

$$
\int \frac{\cos \epsilon}{P Q} d s=\int_{0}^{\theta} \frac{a \cos (\pi-\theta) d \theta}{\left.\sqrt{\left(a^{2}+2 a A\right.} \cos \theta+A^{2}+b^{2}\right)},
$$

an integral composed of incomplete I and II. E. I.'s : and this must be integrated round the circle $A Q B$ to obtain the vector potential $G$ at $P$ perpendicular to the plane $A P B$; and then multiplied by $2 \pi A$ to obtain $M$ for the two circles in parallel planes.

According to our method of leaving the algebraical expression intact, we first substitute

$$
P Q^{2}=a^{2}+2 a A \cos \theta+A^{2}+b^{2}=m^{2}\left(t_{1}-t\right),
$$

and supposing $t=t_{3}$ and $t_{2}$ at $A$ and $B$, where $\theta=0$ and $\pi$,

so that

$$
\begin{aligned}
P A^{2}=r_{1}^{2}=(a+A)^{2}+b^{2} & =m^{2}\left(t_{1}-t_{3}\right), \quad P B^{2}=r_{2}^{2}=(a-A)^{2}+b^{2}=m^{2}\left(t_{1}-t_{2}\right), \\
P Q^{2}-r_{2}^{2} & =2 a A(1+\cos \theta)=m^{2}\left(t_{2}-t\right), \\
r_{1}^{2}-P Q^{2} & =2 a A(1-\cos \theta)=m^{2}\left(t-t_{3}\right),
\end{aligned}
$$

$$
\begin{aligned}
4 a A \cos \theta & =m^{2}\left(t_{2}+t_{3}-2 t\right), \quad r_{1}^{2}-r_{2}^{2}=4 a A=m^{2}\left(t_{2}-t_{3}\right), \\
\frac{r_{2}}{r_{1}} & =\sqrt{\frac{t_{1}-t_{2}}{t_{1}-t_{3}}}=c^{\prime}, \quad \frac{2 \sqrt{ }(a A)}{r_{1}}=\sqrt{\frac{t_{2}-t_{3}}{t_{1}-t_{3}}}=c ;
\end{aligned}
$$




$$
\begin{gathered}
\theta=2 \omega=2 \tan ^{-1} \sqrt{\frac{t-t_{3}}{t_{2}-t}}, d \theta=\frac{d t}{\sqrt{ }\left(t_{2}-t \cdot t-t_{3}\right)}, \frac{d \theta}{P Q}=\frac{2}{r_{1}} \frac{V\left(t_{1}-t_{3}\right) d t}{\sqrt{ } T} \\
M=\int_{0}^{2 \pi} \frac{2 \pi a A \cos (\pi-\theta) d \theta}{P Q}=2 \pi r_{1} \int_{t_{3}}^{t_{2}} \frac{2 t-t_{2}-t_{3}}{\sqrt{ }\left(t_{1}-t_{3}\right)} \frac{d t}{\sqrt{ } T} \\
=4 \pi r_{1} \int \frac{t-t_{3}}{\sqrt{ }\left(t_{1}-t_{3}\right)} \frac{d t}{\sqrt{ } T}-2 \pi r_{1} \frac{t_{2}-t_{3}}{t_{1}-t_{3}} \int \frac{\sqrt{ }\left(t_{1}-t_{3}\right) d t}{\sqrt{ } T}
\end{gathered}
$$

and then by $(10) \S 3$ and $(1),(4) \S 2$,

$$
M=2 \pi r_{1}\left[2(F-E)-c^{2} F^{\prime}\right],
$$

as in MAXwELL, with the original sign changed; and by the quadric transformation employing $(10) \S 18$

$$
M=8 \pi r_{1} \frac{K-H}{1+k}=4 \pi\left(r_{1}+r_{2}\right)(K-H)=8 \pi V(a A) \frac{K-H}{\sqrt{ } k},
$$

doubling MAXweLL's original result, and to be divided by $10^{9}$ to bring it to henries.

Maxwell's modular angle $\gamma$ is now seen to be $A E B$ in fig. $A$ where $A E$ is the tangent from $A$ to the circle on the diameter $C C^{\prime}$, where $P C, P C^{\prime}$ are the bisectors of the angle $A P B$; and then $B E$ is at right angles to $A B$, so that $A, B$ being limiting points of this circle

$$
\cos \gamma=\frac{E B}{E A}=\frac{P B}{P A}=\frac{r_{2}}{r_{1}}=c^{\prime} .
$$

Putting $k=\sin \beta$, so that $\beta$ is the modular angle of the period $K$ and $K^{\prime}$,

$$
\sin \beta=\frac{r_{1}-r_{2}}{r_{1}+r_{2}}=\frac{A C-C B}{A C+C B}=\frac{O C}{O A}=\frac{A C}{A C^{\prime \prime}},
$$

so that $\beta$ is the angle $O C^{\prime} D$ in fig. $B$ where $C D$ is perpendicular to $A B$, and $C^{\prime} D$ the tangent at $D$.

27. A geometrical interpretation can be given in LANDEN's manner of these transformations in the plane $A Q B$ of the circular disc or wire in fig. $B$, as well as in the perpendicular plane $A P B$ of fig. $A$, in which $P C, P C^{\prime}$, the bisectors of the angle $A P B$, are axes of the elliptic cone whose vertex is $P$ and circular base $A Q B$.

The point $F$ in fig. $A$ where $P C$ meets the axis of the circle $A Q B$ is the center of a sphere of which $C$ and $P$ are limiting points, so that $P Q / Q C$ is a constant ratio.

Now, $\omega$ denoting the angle $A B Q$ in fig. $B$,

$$
P Q^{2}=r_{1}^{2} \cos ^{2} \omega+r_{2}^{2} \sin ^{2} \omega,
$$


and at $D$, where $P D C^{\prime}$ is the tangent plane of the cone,

$$
\begin{aligned}
& \cos 2 \omega=-\frac{O C}{O B}=-\frac{r_{1}-r_{2}}{r_{1}+r_{2}}, \\
& \cos ^{2} \omega=\frac{r_{2}}{r_{1}+r_{2}}, \quad \sin ^{2} \omega=\frac{r_{1}}{r_{1}+r_{2}},
\end{aligned}
$$

(3) $A B D=\operatorname{am} \frac{1}{2} F, \quad P D^{2}=r_{1} r_{2}, \quad C D^{2}=A C \cdot C B=\frac{4 r_{1} r_{2} a^{2}}{\left(r_{1}+r_{2}\right)^{2}}$, so that, round the circle $A Q B$,

and so also

$$
\frac{P Q}{Q C}=\frac{P D}{D C}=\frac{r_{1}+r_{2}}{2 a},
$$

$$
\frac{P Q}{Q C^{\prime}}=\frac{r_{1}-r_{2}}{2 a}, \quad \frac{Q C}{Q C^{\prime}}=\frac{r_{1}-r_{2}}{r_{1}+r_{2}}=k .
$$

On fig. $B$ the angles $Q C A, Q C^{\prime} A$ or $C Q O$ are denoted by $\chi, \chi^{\prime}$, and $Q B A, Q^{\prime} B A$ by $\omega, \omega^{\prime}$; and we put, as in $\S 16$,

$$
\begin{gathered}
\chi=\operatorname{am} 2 h K, \quad \omega=\operatorname{am} h F, \quad \omega^{\prime}=\operatorname{am}(1-h) F, \\
P Q=r_{1} \operatorname{dn} h F, \quad C Q=\frac{2 a r_{1}}{r_{1}+r_{2}} \operatorname{dn} h F^{\prime}=C A \operatorname{dn} h F,
\end{gathered}
$$

and then, since $O Q C=\chi^{\prime}, O Q C^{\prime}=\chi, \sin \chi^{\prime}=k \sin \chi, \cos \chi^{\prime}=\operatorname{dn} 2 h K$,

(8) $C Q, C Q^{\prime}=a \cos \chi^{\prime} \pm a k \cos \chi=a(\operatorname{dn} 2 h K \pm k \operatorname{cn} 2 h K)$,

(9) $P Q, P Q^{\prime}=\frac{1}{2}\left(r+r_{2}\right)\left(\cos \chi^{\prime} \pm k \cos \chi\right), \quad P Q \cdot P Q^{\prime}=r_{1} r_{2}$,

(16) $\sin \omega=\frac{1}{2} V\left(1+\sin \chi \cdot 1+\sin \chi^{\prime}\right)-\frac{1}{2} V\left(1-\sin \chi \cdot 1-\sin \chi^{\prime}\right)$, etc., the geometrical equivalent of the LANDEN transformation in $\S 16$. 
28. In the transformation of $M$ to MAxweLL's second form in $\S 702$, we notice that, from elementary geometrical considerations,

(1) $\frac{a d \theta}{C Q}=\frac{d \chi}{\cos \chi^{\prime}}, \quad \frac{a d \theta}{P Q}=\frac{2 a}{r_{1}+r_{2}} \frac{d \chi}{\cos \chi^{\prime}}, \quad \sin \chi^{\prime}=k \sin \chi, \quad \cos \chi^{\prime}=\Delta \chi$,

$$
\begin{aligned}
M & =\int_{0}^{2 \pi} \frac{-2 \pi a A \cos \theta d \theta}{P Q}=\frac{-8 \pi a A}{r_{1}+r_{2}} \int_{0}^{\pi} \cos \left(\chi+\chi^{\prime}\right) \frac{d \chi}{\cos \frac{\chi}{\chi^{\prime}}} \\
& =\frac{8 \pi a A}{r_{1}+r_{2}} \int\left(\frac{k \sin ^{2} \chi}{\cos \chi^{\prime}}-\cos \chi\right) d \chi \\
& =\frac{8 \pi a A}{r_{1}-r_{2}} \int_{0}^{\pi}\left(\frac{1-\Delta^{2} \chi}{\Delta \chi}-k \cos \chi\right) d \chi=4 \pi\left(r_{1}+r_{2}\right)(K-H) .
\end{aligned}
$$

Hence another method in place of MAXwELL's $\$ 702$ for drawing the lines of magnetic force $M=$ constant, employing WeIR's Azimuth Diagram covered by confocal conics for which $r_{1} \pm r_{2}$ is constant.

Denoting WeIR's hour angle by $\alpha$ and latitude argle by $\lambda$, then

(3) $\quad \sin \alpha=\frac{r_{1}-r_{2}}{2 a}, \quad \cos \lambda=\frac{2 a}{r_{1}+r_{2}}, \quad k=\sin \alpha \cos \lambda, \quad \frac{M}{8 \pi a}=\frac{K-H}{\cos \lambda}$.

Supposing the curved line $C P C^{\prime}$ in fig. $A$ to represent for a moment a line of magnetic force of constant $M$, starting from $C$ where $\lambda=0$, and orthogonal to the lines of constant $\Omega$, such as those shown radiating from $A$,

$$
k=\sin \alpha, \quad \frac{M}{8 \pi a}=K-H .
$$

At $C^{\prime}$ where $\alpha=90^{\circ}$,

$$
k=\cos \lambda, \quad \frac{M}{8 \pi a}=\frac{K-H}{k} .
$$

To find an intermediate point $P$ on $C P C^{\prime}$ assume an arbitrary value of $k$ and $K-H$, less than the value at $C$, and calculate $\lambda$ and $\alpha$ from the relation

$$
\begin{aligned}
& \cos \lambda=(K-H) \div \frac{M}{8 \pi a}, \\
& \sin \alpha=\frac{k}{\cos \lambda}=\frac{M}{8 \pi a} \div \frac{K-H}{k} .
\end{aligned}
$$

We thus require a table of $K-H$ and $(K-H) / k$, say for every five degrees of the modular angle $\theta=\sin ^{-1} k$; the work of redrawing MaxweLL's figure XVIII of the lines of magnetic force for a circular current is being carried out on this method. 
The meridians and parallels of latitude of a stereographic projection on fig. $A$, with poles at $A$ and $B$, will map out the electromagnetic field of a straight current through $A$ and the return through $B$ perpendicular to the plane, by means of the longitude for magnetic potential, and then $M=2 \alpha=\log \left(r_{1} / r_{2}\right)$.

29. Putting $\cos \theta=c$, the algebraical form of the quadric substitution is

$$
y \text { or } y-y_{3}=m^{2}\left(s-s_{3}\right)=\frac{a^{2} \sin ^{2} \theta}{P Q^{2}}=\sin ^{2} Q P N,
$$

of which the graph is a hyperbola in the coördinates $(c, y)$;

because

$$
\begin{aligned}
& y_{1}-y=m^{2}\left(s_{1}-s\right)=\left[\frac{a\left(c-c_{1}\right)}{P Q}\right]^{2}, \\
& y_{2}-y=m^{2}\left(s_{2}-s\right)=\left[\frac{a\left(c-c_{2}\right)}{P Q}\right]^{2},
\end{aligned}
$$

$$
\begin{aligned}
& a^{2} c^{2}+2 a A y c+\left(a^{2}+A^{2}+b^{2}\right) y-a^{2}=0, \\
& (a c+A y)^{2}=A^{2}\left(y_{1}-y\right)\left(y_{2}-y\right), \\
& y_{1}=\left(\frac{r_{1}+r_{2}}{2 A}\right)^{2}=\left(\frac{2 a}{r_{1}-r_{2}}\right)^{2}, \quad c_{1}=-\frac{A y_{1}}{a}=-\frac{r_{1}+r_{2}}{r_{1}-r_{2}}=-\sqrt{\frac{y_{1}}{y_{2}}}, \\
& y_{2}=\left(\frac{r_{1}-r_{2}}{2 A}\right)^{2}=\left(\frac{2 a}{r_{1}+r_{2}}\right)^{2}, \quad c_{2}=-\frac{\nexists A y_{2}}{a}=-\frac{r_{1}-r_{2}}{r_{1}+r_{2}}=-\sqrt{\frac{y_{2}}{y_{1}}} \text {, } \\
& y_{1} y_{2}=\left(\frac{a}{A}\right)^{2}, \quad c_{1} c_{2}=1 \text {, } \\
& c=\frac{-y+\sqrt{ }\left(y_{1}-y \cdot y_{2}-y\right)}{\sqrt{ }\left(y_{1} y_{2}\right)}, \\
& c-c_{1}=\frac{V\left(y_{1}-y\right)\left[V\left(y_{1}-y\right)+V\left(y_{2}-y\right)\right]}{V\left(y_{1} y_{2}\right)}, \\
& c-c_{2}=\frac{V\left(y_{2}-y\right)\left[V\left(y_{1}-y\right)+V\left(y_{2}-y\right)\right]}{V\left(y_{1} y_{2}\right)}, \\
& \frac{P Q}{a}=\frac{\sqrt{ }\left(y_{1}-y\right)+\vee\left(y_{2}-y\right)}{\sqrt{ }\left(y_{1} y_{2}\right)}, \quad \frac{C Q}{a}=\frac{\vee\left(y_{1}-y\right)+\sqrt{ }\left(y_{2}-y\right)}{\sqrt{ } y_{1}}, \\
& \frac{P Q}{A}=\vee\left(y_{1}-y\right)+\vee^{\prime}\left(y_{2}-y\right), \\
& \sin \theta=\frac{V y\left[V\left(y_{1}-y\right)-V\left(y_{2}-y\right)\right]}{\sqrt{ }\left(y_{1} y_{2}\right)}, \\
& \tan \frac{1}{2} \theta=\frac{\left(\sqrt{ } y_{1}+\sqrt{ } y_{2}\right) \sqrt{ } y}{\sqrt{ } y_{2} \sqrt{ }\left(y_{1}-y\right)+\sqrt{ } y_{1} \sqrt{ }\left(y_{2}-y\right)} .
\end{aligned}
$$


Taking logarithmic differentials of $\tan \frac{1}{2} \theta$ and $\sin \theta$,

$$
\frac{d \theta}{\sin \theta} \quad \frac{V\left(y_{1} y_{2}\right) d y}{2 y \sqrt{ }\left(y_{1}-y \cdot y_{2}-y\right)}, \quad \cos \theta \frac{d \theta}{\sin \theta}=\frac{d y}{2 y}+\frac{d y}{2 \sqrt{ }\left(y_{1}-y \cdot y_{2}-y\right)}
$$

and multiplying by $\sqrt{ } y=a \sin \theta / P Q$,

$$
\begin{gathered}
\frac{a d \theta}{P Q}=\frac{\sqrt{ }\left(y_{1} y_{2}\right) d y}{\sqrt{ }}, \quad \frac{a \cos \theta d \theta}{P Q}=\frac{d y}{2 \sqrt{ } y}+\frac{y d y}{\sqrt{ } Y}, \\
Y=4 y \cdot y_{1}-y \cdot y_{2}-y
\end{gathered}
$$

and thence the integrals in the algebraical form for determining MaxweLL's second expression for $M$ in terms of $K$ and $H$, noticing that $y$ increases from zero to $y_{2}$ and back again to zero, as $\theta$ increases from 0 to $\pi$.

With the transformation of $(4) \S 25$

$$
\begin{aligned}
& \frac{M}{4 \pi \sqrt{ }(a A)}=\int \frac{2 \operatorname{ch}^{2} \delta-\operatorname{ch}^{2} \zeta}{\operatorname{ch}^{2} \zeta} \frac{d \zeta}{\sqrt{ }\left(\operatorname{ch}^{2} \zeta-\operatorname{ch}^{2} \delta\right)} \\
& =v^{2} \int \frac{2 \operatorname{ch} 2 \delta-\operatorname{ch} 2 \zeta+1}{\operatorname{ch} 2 \zeta+1} \frac{d \zeta}{\sqrt{(\cos 2 \zeta-\operatorname{ch} 2 \delta)}} \\
& =\sqrt{2} \cdot \int^{\infty}\left[\frac{e^{-2 \zeta}}{\sqrt{(\operatorname{ch} 2 \zeta-\operatorname{ch} 2 \delta)}}+\frac{1}{2} \frac{d}{d \zeta} \frac{V(\operatorname{ch} 2 \zeta-\operatorname{ch} 2 \delta)}{e^{2 \zeta}+1}\right] d \zeta,
\end{aligned}
$$

of which the second term vanishes.

Components of electromagnetic force of a circular current.

30. We can now express the components of $M$, and also of the magnetic potential $\Omega$, in terms of $E$ and $F$, or $H$ and $K$, so as to be able to select the simplest form. Thus

$$
\begin{aligned}
\frac{d M}{d b} & =\int_{0}^{2 \pi} \frac{2 \pi a A b \cos \theta d \theta}{P Q^{3}}=-\frac{2 \pi b}{m} \int_{t_{3}}^{t_{2}} \frac{2 t-t_{2}-t_{3}}{t_{1}-t} \frac{d t}{\sqrt{ } T} \\
& =-\frac{2 \pi b}{r_{1}} \int\left(\frac{t_{1}-t_{3}+t_{1}-t_{2}}{t_{1}-t}-2\right) \frac{V\left(t_{1}-t_{3}\right) d t}{V T} \\
& =-\frac{2 \pi b}{r_{1}}\left(\frac{1+c^{\prime 2}}{c^{\prime 2}} E-2 F\right)=-\frac{2 \pi b r_{1}}{r_{2}^{2}}\left[\left(1+c^{\prime 2}\right) E-2 c^{\prime 2} F\right]
\end{aligned}
$$

by a theorem analogous to those in $\S 3$,

$$
\int_{t_{3}}^{t} \frac{t_{1}-t_{2}}{t_{1}-t} \frac{\sqrt{ }\left(t_{1}-t_{3}\right) d t}{\sqrt{ } T}=\int_{0}^{h} \mathrm{dn}^{2}(1-h) F \cdot F d h=h E-\operatorname{zn}(1-h) F .
$$


Or, with

$$
\cos \theta=\cos \left(\chi+\chi^{\prime}\right)=\frac{\left(\cos \chi^{\prime}+k \cos \chi\right)\left(\cos \chi-k \cos \chi^{\prime}\right)}{k^{\prime 2}}
$$

$$
\begin{aligned}
& P Q=\frac{1}{2}\left(r_{1}+r_{2}\right)\left(\cos \chi^{\prime}+k \cos \chi\right), \quad d \theta=\left(\cos \chi^{\prime}+k \cos \chi\right) \frac{d \chi}{\cos \frac{\chi}{\chi^{\prime}},} \\
& \frac{d M}{d b}=\frac{32 \pi a A b}{\left(r_{1}+r_{2}\right)^{3} k^{\prime 2}} \int_{0}^{\pi} \frac{\cos \chi-k \cos \chi^{\prime}}{\cos \chi^{\prime}+k \cos \chi} \frac{d \chi}{\cos \chi^{\prime}}
\end{aligned}
$$

$$
\begin{aligned}
& =\frac{32 \pi a A b}{\left(r_{1}+r_{2}\right)^{3} k^{\prime 4}} \int\left[\left(1+k^{2}\right) \cos \chi \cos \chi^{\prime}-k\left(\cos ^{2} \chi+\cos ^{2} \chi^{\prime}\right)\right] \frac{d \chi}{\cos \frac{\chi^{\prime}}{}} \\
& =-\frac{32 \pi a A b}{\left(r_{1}+r_{2}\right)^{3} k k^{\prime 4}} \int\left[\left(1+k^{2}\right) \cos ^{2} \chi^{\prime}-\left(1-k^{2}\right)\right] \frac{d \chi}{\cos \chi^{\prime}} \\
& =-\frac{16 \pi b}{r_{1}+r_{2}} \frac{\left(1+k^{2}\right) H-\left(1-k^{2}\right) K}{k^{\prime 4}}
\end{aligned}
$$

Next

$$
A \frac{d M}{d A}=M+\int_{0}^{2 \pi} \frac{2 \pi a A \cos \theta\left(A^{2}+a A \cos \theta\right) d \theta}{P Q^{3}}
$$

and similarly

$$
=M+\frac{A^{2}}{b} \frac{d M}{d b}+\int \frac{2 \pi(a A \cos \theta)^{2} d \theta}{P Q^{3}},
$$

$$
a \frac{d M}{d a}=M+\frac{a^{2}}{b} \frac{d M}{d b}+\int \frac{2 \pi(a A \cos \theta)^{2} d \theta}{P Q^{3}}
$$

of which the last integral $C$ is given by

$$
\begin{aligned}
C & =\frac{1}{2} \pi m \int_{t_{2}}^{t_{2}} \frac{\left(t_{2}+t_{3}-2 t\right)^{2}}{t_{1}-t} \frac{d t}{\sqrt{ } T} \\
& =\frac{1}{2} \pi r_{1}\left[\frac{\left(2 t_{1}-t_{2}-t_{3}\right)^{2}}{t_{1}-t_{2} t_{1}-t_{3}} \int \frac{t_{1}-t_{2}}{t_{1}-t} \frac{V\left(t_{1}-t_{3}\right) d t}{\sqrt{ }}\right. \\
& \left.-4 \frac{2 t_{1}-t_{2}-t_{3}}{t_{1}-t_{3}} \int \frac{\sqrt{ }\left(t_{1}-t_{3}\right) d t}{\sqrt{ } T}+4 \int \frac{t_{1}-t}{\sqrt{ }\left(t_{1}-t_{3}\right)} \frac{d t}{\sqrt{ } T}\right] \\
& =\frac{1}{2} \pi r_{1}\left[\frac{\left(1+c^{\prime 2}\right)^{2}}{c^{\prime 2}} E-4\left(1+c^{\prime 2}\right) F+4 E\right]=\frac{1}{2} \pi r_{1} \frac{c^{4}}{c^{\prime 2}} E-M,
\end{aligned}
$$

by preceding theorems on the I. and II. E. I., so that

$$
A \frac{d M}{d A}=\frac{1}{2} \pi r_{1} \frac{c^{4}}{c^{\prime 2}} E+\frac{A^{2}}{b} \frac{d M}{d b}
$$




$$
a \frac{d M}{d a}=\frac{1}{2} \pi r_{1} \frac{c^{4}}{c^{\prime 2}} E+\frac{a^{2}}{b} \frac{d M}{d b}
$$

and the relation

$$
a \frac{d M}{d a}+A \frac{d M}{d A}+b \frac{d M}{d b}=M
$$

is satisfied, required in consequense of $M$ being a homogeneous function of $a, A, b$, of the first degree. Otherwise

$$
\begin{aligned}
& C=\frac{32 \pi a^{2} A^{2}}{\left(r_{1}+r_{2}\right)^{3}} \int_{0}^{\pi} \frac{\left(\cos \chi-k \cos \chi^{\prime}\right)^{2}}{k^{\prime 4}} \frac{d \chi}{\cos \chi^{\prime}} \\
= & \frac{32 \pi a^{2} A^{2}}{\left(r_{1}+r_{2}\right)^{3}} \int \frac{\left(1+k^{4}\right) \cos ^{2} \chi^{\prime}-\left(1-k^{2}\right)-2 k^{3} \cos \chi \cos \chi^{\prime}}{k^{2} k^{\prime 4}} \frac{d \chi}{\cos \chi^{\prime}} \\
= & \frac{64 \pi a^{2} A^{2}}{\left(r_{1}+r_{2}\right)^{3}} \frac{\left(1+k^{4}\right) H-\left(1-k^{2}\right) K}{k^{2} k^{\prime 4}} \\
= & 4 \pi\left(r_{1}+r_{2}\right) \frac{\left(1+k^{4}\right) H-\left(1-k^{2}\right) K}{k^{\prime 4}}, \\
& M+C=4 \pi\left(r_{1}+r_{2}\right) \frac{k^{2}}{k^{\prime 4}}\left(2 H-k^{\prime 2} K\right) .
\end{aligned}
$$

31. If $\Omega$ denotes the magnetic potential or conical angle at $P$ of the circular ring $A Q B$, the magnetic components are (E. and M., § 703),

$$
\frac{d \Omega}{d A}=\frac{1}{2 \pi A} \frac{d M}{d b}=-\frac{4 a b}{r_{1}^{3}} \frac{\left(1+c^{\prime 2}\right) E-2 c^{\prime 2} F}{c^{2} c^{2}}
$$

or

$$
-\frac{32 a b}{\left(r_{1}+r_{2}\right)^{3}} \frac{\left(1+k^{2}\right) H-\left(1-k^{2}\right) K}{k k^{4}},
$$

(2) $\frac{d \Omega}{d b}=-\frac{1}{2 \pi A} \frac{d M}{d A}=-\frac{M+C}{2 \pi A^{2}}-\frac{1}{2 \pi b} \frac{d M}{d b}=-\frac{4 a^{2}}{r_{1}^{3}} \frac{E}{c^{\prime 2}}+\frac{1}{r_{1}} \frac{\left(1+c^{\prime 2}\right) E-2 c^{\prime 2} F}{c^{\prime 2}}$

or

$$
-\frac{32 a^{2}}{\left(r_{1}+r_{2}\right)^{3}} \frac{2 H-k^{\prime 2} K}{k^{\prime 4}}+\frac{3}{r_{1}+r_{2}} \frac{\left(1+k^{2}\right) H-\left(1-k^{2}\right) K}{k^{\prime 4}} .
$$

Also, from the homogeneity of $\Omega$, of zero dimension,

$$
a \frac{d \Omega}{d a}=-A \frac{d \Omega}{d A}-b \frac{d \Omega}{d b}=\frac{b}{2 \pi A^{2}}(M+C)=\frac{4 a^{2} b}{r_{1}^{3}} \frac{E}{c^{\prime 2}}
$$

or

$$
\frac{32 a^{2} b}{\left(r_{1}+r_{2}\right)^{3}} \frac{2 H-k^{2} K}{k^{4}}
$$


In the neighborhood of the axis, where $A, r_{1}-r_{2}, c$, and $k$ are small,

$$
F=\frac{1}{2} \pi\left(1+\frac{c^{2}}{4}+\frac{9 c^{4}}{64} \cdots\right), \quad E=\frac{1}{2} \pi\left(1-\frac{c^{2}}{4}-\frac{3 c^{4}}{64} \cdots\right),
$$

(5) $\quad F-E=\frac{1}{2} \pi\left(\frac{1}{2} c^{2}+\frac{3}{16} c^{4} \cdots\right), \quad E-{c^{\prime}}^{2} F^{\prime}=\frac{1}{2} \pi\left(\frac{1}{2} c^{2}+\frac{1}{1} \frac{3}{6} c^{4} \cdots\right)$,

$$
\frac{d M}{d b}=-\frac{6 \pi^{2} a^{2} A^{2} b}{r_{1}^{5}}, \quad \text { or } \quad-\frac{192 \pi^{2} a^{2} A^{2} b}{\left(r_{1}+r_{2}\right)^{5}}
$$

$$
M+C=\frac{1}{2} \pi r_{1} \frac{16 a^{2} A^{2}}{r_{1}^{4}} \frac{1}{2} \pi\left(1+\frac{3 c^{2}}{4} \cdots\right)=\frac{4 \pi^{2} a^{2} A^{2}}{r_{1}^{3}}\left(1+\frac{3 a A}{r_{1}^{2}}\right)
$$

or

$$
=4 \pi\left(r_{1}+r_{2}\right) \frac{16 a^{2} A^{2}}{\left(r_{1}+r_{2}\right)^{4}} \frac{1}{2} \pi\left(1+\frac{9}{4} k^{2}\right)=\frac{32 \pi^{2} a^{2} A^{2}}{\left(r_{1}+r_{2}\right)^{3}}\left[1+\frac{36 a^{2} A^{2}}{\left(r_{1}+r_{2}\right)^{4}}\right],
$$

$$
\frac{d \Omega}{d A}=\frac{3 \pi a^{2} A b}{r_{1}^{5}}, \quad \text { or } \quad \frac{96 \pi a^{2} A b}{\left(r_{1}+r_{2}\right)^{5}},
$$

$$
\frac{d \Omega}{d b}=\frac{2 \pi a^{2}}{r_{1}^{3}}+\frac{6 \pi a^{3} A}{r_{1}^{5}}, \quad \text { or } \quad \frac{16 \pi a^{2}}{\left(r_{1}+r_{2}\right)^{3}}\left[1+\frac{36 a^{2} A^{2}}{\left(r_{1}+r_{2}\right)^{4}}\right]-\frac{96 \pi a^{2} A^{2}}{\left(r_{1}+r_{2}\right)^{5}}
$$

On the axis itself, $A=0$,

$$
\frac{d \Omega}{d A}=0, \quad \frac{d \Omega}{d b}=\frac{2 \pi a^{2}}{r_{1}^{3}} .
$$

A simpler resolution of the magnetic force is into components $G_{1}$ and $G_{2}$, perpendicular to $P A$ and $P B$, and we find (A. Russell, Philosophical Magazine, April, 1907),

$$
G_{1}=\frac{F-E}{A}=\frac{2}{A}\left(K-\frac{H}{1+k}\right), \quad G_{2}=\frac{E-c^{\prime 2} F}{c^{\prime} A}=\frac{2}{A}\left(\frac{H}{1-k}-K\right),
$$

and on the axis $A=0, G_{1}=G_{2}=\pi a / r_{1}^{2}$, but $G_{1}$ and $G_{2}$ are infinite along the wire.

A similar resolution of the gravitational force of the potential $\int a d \theta / P Q$ of the circular ring $A B$ will show that it can be resolved into the components $2 G_{1}$ and $2 G_{2}$, but now in the direction of $P A$ and $P B$. 
Galvanometer constant of a circular coil.

32. To calculate the galvanometer constant $G$ at a point on the axis of a coil of wire of $n$ turns in the form of a ring of given cross section $\alpha$, we have to evaluate the integral

$$
G=\frac{n}{\alpha} \iint \frac{2 \pi x^{2} d x d y}{\left(x^{2}+y^{2}\right)^{3}}=\frac{2 \pi n}{\alpha} \int \frac{y d x}{\left(x^{2}+y\right)^{\frac{1}{3}}},
$$

taken over the area or round the perimeter.

For a circular section $A Q B$, with the axis of the ring through $C^{\prime}$ in fig. $B$,

$$
G=\frac{2 \pi n}{\pi a^{2}} \int_{0}^{2 \pi} \frac{a^{2} \sin ^{2} \theta d \theta}{C^{\prime} Q}=\int_{0}^{2 \pi} \frac{2 n \sin ^{2} \theta d \theta}{C^{\prime} Q},
$$

and putting as before in $\S 26$, with $O C^{\prime}=a / k$,

(3) $C^{\prime} Q^{2}=m^{2}\left(t_{1}-t\right), \quad C^{\prime} Q^{2}-C^{\prime} B^{2}=\frac{2 a^{2}}{k}(1+\cos \theta)=m^{2}\left(t_{2}-t\right)$,

$$
C^{\prime} A^{2}-C^{\prime} Q^{2}=\frac{2 a^{2}}{k}(1-\cos \theta)=m^{2}\left(t-t_{3}\right),
$$

we have

(4) $m^{2}\left(t_{1}-t_{3}\right)=C^{\prime} A^{2}=a^{2}\left(\frac{1}{k}+1\right)^{2}, \quad m^{2}\left(t_{1}-t_{2}\right)=C^{\prime} B^{\prime \prime}=a^{2}\left(\frac{1}{k}-1\right)$,

$$
c^{\prime}=\sqrt{\frac{t_{1}-t_{2}}{t_{1}-t_{3}}}=\frac{1-k}{1+k}
$$

$$
\begin{aligned}
& G=\frac{2 n k^{2} m^{3}}{a^{4}} \int_{t_{3}}^{t_{2}}\left(t_{2}-t \cdot t-t_{3}\right) \frac{d t}{\sqrt{ } T}
\end{aligned}
$$

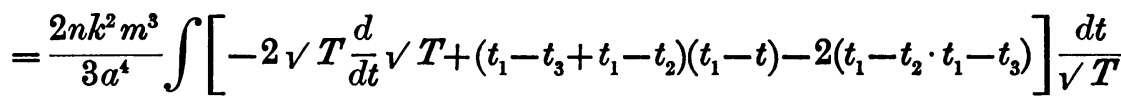

$$
\begin{aligned}
& =\frac{2 n k^{2} m^{3}}{3 a^{4}}\left[\left(t_{1}-t_{3}+t_{1}-t_{2}\right) V\left(t_{1}-t_{3}\right) E-2\left(t_{1}-t_{2}\right) V\left(t_{1}-t_{3}\right) F\right] \\
& =\frac{32 n}{3 A C^{\prime}} \frac{\left(1+c^{\prime 2}\right) E-2{c^{\prime}}^{2} F}{c^{4}} .
\end{aligned}
$$

The reduction can also be carried out with the functions $K$ and $H$, by means of the angles $\chi$ and $\chi^{\prime}$, and 


$$
C^{\prime} Q=\frac{a}{k} \cos \chi^{\prime}+a \cos \chi, \quad \frac{a d \theta}{C^{\prime} Q}=k \frac{d \chi}{\cos \chi^{\prime}},
$$

(8) $G=\frac{4 n k}{a} \int_{0}^{\pi} \sin ^{2}\left(\chi+\chi^{\prime}\right) \frac{d \chi}{\cos \chi^{\prime}}=\frac{8 n}{3 O C^{\prime}} \frac{\left(1+k^{2}\right) H-\left(1-k^{2}\right) K}{k^{2}}$,

and $G$ is given by the same expression when $C^{\prime}$ is moved to any point on the axis of the tore, the modular angle $\beta$ of $K$ and $H$ being half the angle between the tangents from $C^{\prime}$ to the circular section of the tore.

33. The form of the result shows that the integral for $G$ and $d M / d b$ is the same essentially ; for the comparison of these and other integrals it is convenient at this stage to have the following integrals to quote in the sequel:

$$
\int_{0}^{2 \pi} \frac{a d \theta}{P Q}=\frac{4 a F}{r_{1}}=\frac{8 a K}{r_{1}+r_{2}},
$$

the potential of the ring $A B$; and as in $\S 31$,

$$
\begin{aligned}
& \text { (2) } \int \frac{2 a A(1+\cos \theta) d \theta}{P Q^{3}}=\frac{4}{r_{1}} \int_{t_{3}}^{t_{2}} \frac{t_{2}-t}{t_{1}-t} \frac{V\left(t_{1}-t_{3}\right) d t}{V T}=\frac{4}{r_{1}}(F-E)=4 A \frac{G_{1}}{r_{1}} \\
& \text { (3) } \int \frac{2 a A(1-\cos \theta) d \theta}{P Q^{3}}=\frac{4}{r_{1}} \int \frac{t-t_{3}}{t_{1}-t} \frac{V\left(t_{1}-t_{3}\right) d t}{V T}=\frac{4}{r_{1}} \frac{E-{c^{\prime 2} F}^{\prime 2}}{c^{\prime 2}}=4 A \frac{G_{2}}{r_{2}} .
\end{aligned}
$$

Since

$$
\begin{aligned}
\frac{d}{d \theta} \frac{2 a A \sin \theta}{P Q} & =\frac{2 a A \cos \theta}{P Q}+\frac{2 a^{2} A^{2} \sin ^{2} \theta}{P Q^{3}} \\
& =\frac{P Q^{2}-\frac{1}{2}\left(r_{1}^{2}+r_{2}^{2}\right)}{P Q}+\frac{\left(r_{1}^{2}-P Q^{2}\right)\left(P Q^{2}-r_{2}^{2}\right)}{2 P Q^{3}} \\
& =\frac{1}{2} P Q-\frac{1}{2} \frac{r_{1}^{2} r_{2}^{2}}{P Q^{3}},
\end{aligned}
$$

Also

$$
\begin{aligned}
\frac{d}{d \theta}(2 a A \sin \theta P Q) & =\left(P Q^{2}-\frac{r_{1}^{2}+r_{2}^{2}}{2}\right) P Q-\frac{\left(r_{1}^{2}-P Q^{2}\right)\left(P Q^{2}-r_{2}^{2}\right)}{2 P Q} \\
& =\frac{3}{2} P Q^{3}-\left(r_{1}^{2}+r_{2}^{2}\right) P Q+\frac{r_{1}^{2} r_{2}^{2}}{2 P Q}
\end{aligned}
$$




$$
\begin{aligned}
3 \int_{0}^{2 \pi} P Q^{3} d \theta & =2\left(r_{1}^{2}+r_{2}^{2}\right) \int P Q d \theta-r_{1}^{2} r_{2}^{2} \int \frac{d \theta}{P Q} \\
& =8 r_{1}\left(r_{1}^{2}+r_{2}^{2}\right) E-4 r_{1} r_{2}^{2} F^{\prime} \\
& =4 r_{1}^{3}\left[2\left(1+c^{\prime 2}\right) E-c^{\prime 2} F^{\prime}\right] \\
& =\left(r_{1}+r_{2}\right)^{3}\left[4\left(1+k^{2}\right) H-\frac{1}{2} k^{\prime 2}\left(5+3 k^{2}\right) K\right],
\end{aligned}
$$

$$
3 \int \frac{\sin ^{2} \theta d \theta}{P Q}=\frac{16}{r_{1}} \frac{\left(1+{c^{\prime 2}}^{2}\right) E-2{c^{\prime 2}}^{2}}{c^{4}}=\frac{8}{r_{1}+r_{2}} \frac{\left(1+k^{2}\right) H-\left(1-k_{2}^{2}\right) K}{k^{2}},
$$

(10) $3 \int P Q \cos \theta d \theta=4 r_{1} \frac{\left(1+c^{2}\right) E-2 c^{\prime 2} F}{c^{2}}=2\left(r_{1}+r_{2}\right) \frac{\left(1+k^{2}\right) H-\left(1-k^{2}\right) K}{k}$,

so that as is evident from (7) these last two integrals (9) and (10) are essentially the same as well as the integral (1) $\S 30$; and this shows that $G$ is also the potential of the tore at any point $C^{\prime}$ of its axis, the tore being homogeneous and of mass $2 \pi n$.

Potential of an elliptic disc, gravitational and magnetic.

34. Begin now with the potential $V$ of an elliptic disc

$$
\frac{x^{2}}{a^{2}}+\frac{y^{2}}{b^{2}}+\frac{z^{2}}{0}=1
$$

of uniform surface density 1 ; then (CAYley, Proceedings London Mathematical Society, vol. 6, p. 42)

$$
V=2 a b \int_{\lambda}^{\infty} V\left(1-\frac{x^{2}}{a^{2}+\epsilon}-\frac{y^{2}}{b^{2}+\epsilon}-\frac{z^{2}}{\epsilon}\right) \frac{d \epsilon}{\sqrt{ }\left(a^{2}+\epsilon \cdot b^{2}+\epsilon \cdot \epsilon\right)},
$$

where $\lambda$ is the positive root of

$$
1-\frac{x^{2}}{a^{2}+\epsilon}-\frac{y^{2}}{b^{2}+\epsilon}-\frac{z^{2}}{\epsilon}=0
$$

$\mu$ and $\nu$ denoting the other two.

Next by differentiation of $V$ with respect to $z$ the magnetic potential $\Omega$ is obtained of the elliptic disc, magnetized normally with uniform unit intensity, as represented by the component attraction of the disc perpendicular to the plane, or by the solid or conical angle subtended at a point; so that

$$
\Omega=-\frac{d V}{d z}=\int_{\lambda}^{\infty} \frac{2 a b z d \epsilon}{\epsilon \sqrt{\left(1-\frac{x^{2}}{a^{2}+\epsilon}-\frac{y^{2}}{b^{2}+\epsilon}-\frac{z^{2}}{\epsilon}\right) V\left(a^{2}+\epsilon \cdot b^{2}+\epsilon \cdot \epsilon\right)},}
$$

reducing to 


$$
\begin{gathered}
\Omega=\int_{\lambda}^{\infty} \frac{4 a b z d \epsilon}{\epsilon \sqrt{ }(4 \cdot \epsilon-\lambda \cdot \epsilon-\mu \cdot \epsilon-\nu)}=\int_{\lambda}^{\infty} \frac{4 \sqrt{ }(\lambda \mu \nu)}{\epsilon} \frac{d \epsilon}{\sqrt{E}}, \\
E=4 \cdot \epsilon-\lambda \cdot \epsilon-\mu \cdot \epsilon-\nu .
\end{gathered}
$$

This is proved by determining $x, y, z$ in terms of $\lambda, \mu, \nu$, the elliptic coördinates, such that $(x, y, z)$ is a point of intersection of the three confocals of (3) to the elliptic disc given in (1), when

$$
\begin{gathered}
\epsilon=\lambda, \mu, \nu, \\
\infty>\lambda>0>\mu>-b^{2}>\nu>-a^{2}>-\infty,
\end{gathered}
$$

and then

(8) $x^{2}=\frac{a^{2}+\lambda \cdot a^{2}+\mu \cdot a^{2}+\nu}{a^{2}-b^{2} \cdot a^{2}-0}, \quad y^{2}=\frac{b^{2}+\lambda \cdot b^{2}+\mu \cdot b^{2}+\nu}{b^{2}-a^{2} \cdot b^{2}-0}, \quad z^{2}=\frac{0+\lambda \cdot 0+\mu \cdot 0+\nu}{a^{2}-0 \cdot b^{2}-0}$,

$$
1-\frac{x^{2}}{a^{2}+\epsilon}-\frac{y^{2}}{b^{2}+\epsilon}-\frac{z^{2}}{\epsilon}=\frac{\epsilon-\lambda \cdot \epsilon-\mu \cdot \epsilon-\nu}{a^{2}+\epsilon \cdot b^{2}+\epsilon \cdot \epsilon} .
$$

Interpreted geometrically, $\Omega$ is the solid or conical angle subtended by the elliptic disc (1), or the apparent area, as it may be called; and ScHwARz has shown that the apparent area or Scheinbare Grösse of the ellipsoid or of any elliptic section of the tangent cone is given by an expression equally simple (Göttinger Nachrichten, 1885),

$$
\Omega=\int_{\lambda}^{\infty} \frac{2 V\left(-4 \cdot \lambda_{0}-\lambda \cdot \lambda_{0}-\mu \cdot \lambda_{0}-\nu\right) d \epsilon}{\left(\epsilon-\lambda_{0}\right) V(4 \cdot \epsilon-\lambda \cdot \epsilon-\mu \cdot \epsilon-\nu)}=2 \pi-\int_{\nu}^{\mu} \frac{2 \sqrt{ }\left(-E_{0}\right)}{\lambda_{0}-\epsilon} \frac{d \epsilon}{\sqrt{E}}
$$

where the ellipsoid is defined by

$$
\left(E_{0}=4 \cdot \lambda_{0}-\lambda \cdot \lambda_{0}-\mu \cdot \lambda_{0}-\nu\right) \text {, }
$$

$$
\frac{x^{2}}{a^{2}+\lambda_{0}}+\frac{y^{2}}{b^{2}+\lambda_{0}}+\frac{z^{2}}{\lambda_{0}}=1
$$

reducing to the elliptic disc as its focal ellipse when $\lambda_{0}=0$.

Put and

$$
\epsilon-\lambda=M^{2}\left(s-s_{1}\right), \quad \epsilon-\mu=M^{2}\left(s-s_{2}\right), \quad \epsilon-\nu=M^{2}\left(s-s_{3}\right)
$$

and then, as in (3), 55 ,

$$
\begin{gathered}
\epsilon\left(\text { or } \epsilon-\lambda_{0}\right)=M^{2}(s-\sigma), \\
E=4 \cdot \epsilon-\lambda \cdot \epsilon-\mu \cdot \epsilon-\nu=M^{6} S, \\
4 a^{2} b^{2} z^{2}=4 \lambda \mu \nu=-E_{0}=-M^{6} \Sigma ;
\end{gathered}
$$

$$
\Omega=\int_{s_{1}}^{\infty} \frac{2 \sqrt{ }-\Sigma}{s-\sigma} \frac{d s}{\sqrt{S}}=4 A=2 \pi-4 B .
$$


35. Schwarz's theorem is proved by considering the equation of the tangent cone from $(x, y, z)$ to the ellipsoid defined by $\epsilon=\lambda_{0}$, which, when referred to its principal axes, the normals of the three confocals through $(x, y, z)$ defined by $\epsilon=\lambda, \mu, \nu$ becomes (Salmon, Solid Geometry, $\S 173$ ),

$$
\frac{x^{2}}{\lambda-\lambda_{0}}+\frac{y^{2}}{\mu-\lambda_{0}}+\frac{z^{2}}{\nu-\lambda_{0}}=0
$$

one of a family of cones confocal for different values of $\lambda_{0}$.

The conical angle $\Omega$ of this tangent cone, and the perimeter $\Phi$ of the spheroconic on unit sphere of the reciprocal cone

$$
\left(\lambda-\lambda_{0}\right) x^{2}+\left(\mu-\lambda_{0}\right) y^{2}+\left(\nu-\lambda_{0}\right) z^{2}=0
$$

are connected by the relation

$$
\Omega+\Phi=2 \pi .
$$

On the reciprocal cone replacing $x, y, z$ by direction cosines $l, m, n$, we may take

subject to the condition

$$
\begin{aligned}
& l^{2}=k(\mu-\nu)\left(\frac{1}{\lambda-\lambda_{0}}-\frac{1}{\epsilon-\lambda_{0}}\right), \\
& m^{2}=k(\nu-\lambda)\left(\frac{1}{\mu-\lambda_{0}}-\frac{1}{\epsilon-\lambda_{0}}\right), \\
& n^{2}=k(\lambda-\mu)\left(\frac{1}{\nu-\lambda_{0}}-\frac{1}{\epsilon-\lambda_{0}}\right),
\end{aligned}
$$

and now

$$
\begin{gathered}
l^{2}+m^{2}+n^{2}=k\left(\frac{\mu-\nu}{\lambda-\lambda_{0}}+\frac{\nu-\lambda}{\mu-\mu_{0}}+\frac{\lambda-\mu}{\nu-\nu_{0}}\right)=1, \\
k=-\frac{\lambda-\lambda_{0} \cdot \mu-\lambda_{0} \cdot \nu-\lambda_{0}}{\mu-\nu \cdot \nu-\lambda \cdot \lambda-\mu}
\end{gathered}
$$

$$
\begin{gathered}
(d \Phi)^{2}=d l^{2}+d m^{2}+d n^{2}=\frac{-E_{0}}{4\left(\epsilon-\lambda_{0}\right)^{2}} \frac{d \epsilon^{2}}{E} \\
E=4 \cdot \epsilon-\lambda \cdot \epsilon-\mu \cdot \epsilon-\nu, \quad E_{0}=4 \cdot \lambda_{0}-\lambda \cdot \lambda_{0}-\mu \cdot \lambda_{0}-\nu .
\end{gathered}
$$

As a point travels round a quadrant of the sphero-conic, $\epsilon$ decreases from $\mu$ to $\nu$, and

$$
\begin{gathered}
\lambda>\lambda_{0}>0>\mu>\epsilon>\nu, \\
\Phi=4 \int_{\nu}^{\mu} \frac{\frac{1}{2} V\left(-E_{0}\right)}{\lambda_{0}-\epsilon} \frac{d \epsilon}{\sqrt{E}}, \\
\Omega=2 \pi-\Phi=4 \int_{\lambda}^{\infty} \frac{\frac{1}{2} V\left(-E_{0}\right)}{\epsilon-\lambda_{0}} \frac{d \epsilon}{\sqrt{E}} .
\end{gathered}
$$

in accordance with (5) $\S 5, \Omega$ and $\Phi$ being $4 A$ and $4 B$, Schwarz's result. 
36. In the notation of Gauss (Cayley, Proceedings of the London Mathematical Society, vol. 6 ; HaLPHen, Fonctions Elliptiques, vol. 2, chap. 8)

$$
G, G^{\prime \prime}, G^{\prime}=\lambda,-\mu,-\nu,
$$

and in the region $\mu>\epsilon>\nu$ we can put

$$
\begin{gathered}
G+G^{\prime} \cos ^{2} T+G^{\prime \prime} \sin ^{2} T=\lambda-\epsilon, \\
G^{\prime} \cos ^{2} T+G^{\prime \prime} \sin ^{2} T=\lambda_{0}-\epsilon, \\
\left(G^{\prime}-G^{\prime \prime}\right) \cos ^{2} T=\mu-\epsilon,\left(G^{\prime}-G^{\prime \prime}\right) \sin ^{2} T=\epsilon-\nu, \\
d T=\frac{d \epsilon}{\sqrt{(4 \cdot \mu-\epsilon \cdot \epsilon-\nu)},} \\
U=\int_{0}^{T} \frac{d T}{\left.\sqrt{\left(G+G^{\prime} \cos ^{2}\right.} T+G^{\prime \prime} \sin ^{2} T\right)}=\int_{-\nu}^{\epsilon} \frac{d \epsilon}{\sqrt{E}}, \\
U V(\lambda-\nu)=h K, \quad T=\operatorname{am} h K .
\end{gathered}
$$

In the region $\infty>\epsilon>\lambda>\lambda_{0}$, we should take

$$
\begin{gathered}
\sin ^{2} T=\frac{\lambda-\nu}{\epsilon-\nu}, \quad \cos ^{2} T=\frac{\epsilon-\lambda}{\epsilon-\nu}, \quad T=\operatorname{am} h K, \\
G+G^{\prime} \cos ^{2} T+G^{\prime \prime} \sin ^{2} T=\frac{\lambda-\nu \cdot \epsilon-\mu}{\epsilon-\nu}, \\
G^{\prime} \cos ^{2} T+G^{\prime \prime} \sin ^{2} T=\frac{\left(\lambda_{0}-\nu \cdot \epsilon-\nu\right)-(\lambda-\nu \cdot \mu-\nu)}{\epsilon-\nu},
\end{gathered}
$$

equivalent to the substitution

37. The inequalities

$$
\left(\epsilon-\nu, \frac{\lambda-\nu \cdot \mu-\nu}{\epsilon-\nu}\right)
$$

$$
\begin{aligned}
& \infty>\epsilon>\lambda>\lambda_{0}>0>\mu>-b^{2}>\nu>-a^{2}, \\
& \infty>s>s_{1}>\sigma>s_{2}>s>s_{3}>-\infty,
\end{aligned}
$$

show that the form $A\left(f K^{\prime}\right)$ or $B\left(f K^{\prime}\right)$ is required; and from (3) $\S 5$,

$$
\Omega=2 \pi(1-f)-4 K \operatorname{zn} f K^{\prime},
$$

equivalent to NAGAOKA's result for a circle expressed by Weierstrass functions; and here, as in $(6),(7),(8),(9) \S 5$, 


$$
\begin{gathered}
\text { zn } f K^{\prime}=Z f K^{\prime}=E \phi-f E, \\
f K^{\prime}=F\left(\phi, k^{\prime}\right), \quad \phi=\operatorname{am} f K^{\prime},
\end{gathered}
$$

so that LEGENDRE's Table IX can be employed for a numerical application,

$$
\begin{gathered}
\sin ^{2} \phi=\operatorname{sn}^{2} f K^{\prime}=\frac{s_{1}-\sigma}{s_{1}-s_{2}}=\frac{\lambda}{\lambda-\mu}, \\
\cos ^{2} \phi=\operatorname{cn}^{2} f K^{\prime}=\frac{\sigma-s_{2}}{s_{1}-s_{2}}=\frac{-\mu}{\lambda-\mu}, \\
\Delta^{2} \phi=\operatorname{dn}^{2} f K^{\prime}=\frac{\sigma-s_{3}}{s_{1}-s_{3}}=\frac{-\nu}{\lambda-\nu}, \\
k^{2}=\frac{s_{2}-s_{3}}{s_{1}-s_{3}}=\frac{\mu-\nu}{\lambda-\nu}, \quad k^{\prime 2}=\frac{s_{1}-s_{2}}{s_{1}-s_{3}}=\frac{\lambda-\mu}{\lambda-\nu},
\end{gathered}
$$

and the modular angle is then half the angle between the generating lines of the confocal hyperboloid of one sheet, or the focal lines of the tangent cone.

38. Thus for instance, if we take $f=\frac{1}{2}$,

$$
\Delta^{2} \phi=\frac{-\nu}{\lambda-\nu}=k=\sqrt{\frac{\mu-\nu}{\lambda-\nu}}, \quad \frac{1}{\lambda}+\frac{1}{\mu}-\frac{1}{\nu}=0, \quad k=\frac{-\mu}{\lambda},
$$

and now

$$
\mu=-k \lambda, \quad v=-\frac{k \lambda}{1-k},
$$

$$
\text { zn } \frac{1}{2} K^{\prime}=\frac{1}{2}(1-k), \quad \Omega=\pi-2(1-k) K .
$$

At an infinite distance,

(3) $\lambda=\infty, \quad \sin \phi=1, \quad k=0, \quad K=\frac{1}{2} \pi, \quad k^{\prime}=1, \quad K^{\prime}=\infty, \quad E^{\prime}=1$,

so that

$$
\text { zn } f K^{\prime}=E \phi-f E^{\prime}=\sin \phi-f=1-f,
$$

a verification.

$$
\Omega=2 \pi(1-f)-2 \pi(1-f)=0,
$$

Inside the focal ellipse, in its plane,

$$
\lambda=0, \quad f=0, \quad \Omega=2 \pi ;
$$

but in the plane outside the ellipse

$$
\mu=0, \quad f=1, \quad \text { zn } K^{\prime}=0, \quad \Omega=0,
$$

to serve as a verification. 
Along the edge of the focal ellipse

$$
\begin{gathered}
\lambda=0, \quad \mu=0, \quad k^{\prime}=0, \quad \text { zn } f K^{\prime}=0, \\
\Omega=2 \pi(1-f),
\end{gathered}
$$

and two surfaces of constant $\Omega_{1}$ and $\Omega_{2}$ intersect along the edge at an angle

$$
\frac{1}{2}\left(\Omega_{2}-\Omega_{1}\right)=\pi\left(f_{1}-f_{2}\right) ;
$$

see Maxwell, E. and M., $§ 487$.

Along the focal hyperbola

$$
\begin{gathered}
\mu=\nu=-b^{2}, \quad k=0, \quad K=\frac{1}{2} \pi, \\
k^{\prime}=1, \quad E^{\prime}=1, \quad E \phi=\sin \phi, \\
\text { zn } f K^{\prime}=\sin \phi-f,
\end{gathered}
$$

so that

$$
\Omega=2 \pi(1-\sin \phi)=2 \pi\left(1-\sqrt{\frac{\lambda}{\lambda-\mu}}\right),
$$

and the conical angle is now bounded by a cone of revolution of vertical angle $\pi-2 \phi$ (CAYley, Proceedings of the London Mathematical Society, vol. 6).

On the axis perpendicular to the plane of the ellipse

$$
\begin{gathered}
x=0, \quad y=0, \quad z^{2}=\lambda, \quad b^{2}+\mu=0, \quad a^{2}+\nu=0, \\
k^{2}=\frac{a^{2}-b^{2}}{a^{2}+z^{2}}, \quad k^{\prime 2}=\frac{b^{2}+z^{2}}{a^{2}+z^{2}}, \\
\operatorname{sn}^{2} f K^{\prime}=\frac{z^{2}}{b^{2}+z^{2}}, \quad \operatorname{cn}^{2} f K^{\prime}=\frac{b^{2}}{b^{2}+z^{2}}, \quad \operatorname{dn}^{2} f K^{\prime}=\frac{a^{2}}{a^{2}+z^{2}}, \\
\operatorname{dn}^{2}(1-f) K^{\prime}=1-\frac{b^{2}}{a^{2}}=e^{2} .
\end{gathered}
$$

39. The equipotential surface $\Omega=2 \pi f$, a constant, cuts the plane of the ellipse (or any other area whatever) along its edge at a constant angle $\pi f$; this is evident from simple geometrical considerations of the conical angle or apparent area of the ellipse as seen from a point close to the edge, when the apparent area on the unit sphere is cut out by two diametral planes, one parallel to the plane of the area, and the other drawn through the adjacent tangent of the area.

Magnetic interpretation of the potential of the elliptic disc.

40. In the potential of an elliptic disc, as given by CAYLEY,

$$
V=4 a b \int_{\lambda}^{\infty}\left(1-\frac{z^{2}}{\epsilon}-\frac{y^{2}}{b^{2}+\epsilon}-\frac{x^{2}}{a^{2}+\epsilon}\right) \frac{d \epsilon}{\sqrt{ } E},
$$


when modified by (9) $\S 34$, and employing (12) $\S 34$, with

$$
\epsilon=m^{2}(s-\sigma), \quad b^{2}+\epsilon=m^{2}\left(s-\sigma^{\prime}\right), \quad a^{2}+\epsilon=m^{2}\left(s-\sigma^{\prime \prime}\right),
$$
$\infty>s>s_{1}>\sigma>s_{2}>\sigma^{\prime}>s_{3}>\sigma^{\prime \prime}>-\infty$,

$$
\lambda=m^{2}\left(s_{1}-\sigma\right) \text {, }
$$$$
b^{2}+\lambda=m^{2}\left(s_{1}-\sigma^{\prime}\right),
$$$$
a^{2}+\lambda=m^{2}\left(s_{1}-\sigma^{\prime \prime}\right),
$$

$$
\begin{array}{lll}
\mu=-m^{2}\left(\sigma-s_{2}\right), & b^{2}+\mu=-m^{2}\left(s_{2}-\sigma^{\prime}\right), & a^{2}+\mu=m^{2}\left(s_{2}-\sigma^{\prime \prime}\right), \\
\nu=-m^{2}\left(\sigma-s_{1}\right), & b^{2}+\nu=-m^{2}\left(\sigma^{\prime}-s_{3}\right), & a^{2}+\nu=m^{2}\left(s_{3}-\sigma^{\prime \prime}\right),
\end{array}
$$

$$
a^{2} b^{2} z^{2}=\lambda \mu \nu=-\frac{1}{4} m^{6} \Sigma,
$$

$$
\left(a^{2}-b^{2}\right) b^{2} y^{2}=b^{2}+\lambda \cdot b^{2}+\mu \cdot b^{2}+\nu=\frac{1}{4} m^{6} \Sigma^{\prime},
$$$$
\left(a^{2}-b^{2}\right) a^{2} x^{2}=a^{2}+\lambda \cdot a^{2}+\mu \cdot a^{2}+\nu=-\frac{1}{4} m^{6} \Sigma^{\prime \prime},
$$

and the terms in $V$ are

$$
\begin{aligned}
4 a b \int_{\lambda}^{\infty} \frac{d \epsilon}{\sqrt{E}} & =\frac{4 a b K}{\sqrt{(\lambda-\nu)}}, \\
4 a b z^{2} \int \frac{d \epsilon}{\epsilon \sqrt{E}} & =4 z \int_{\delta_{1}}^{\infty} \frac{\frac{1}{2} V(-\Sigma)}{s-\sigma} \frac{d s}{\sqrt{S}} \\
& =4 z A\left(f K^{\prime}\right)=4 z\left[\frac{1}{2} \pi(1-f)-K \mathrm{zn} f K^{\prime}\right],
\end{aligned}
$$$$
4 a b y^{2} \int \frac{d \epsilon}{\left(b^{2}+\epsilon\right) \sqrt{ } E}=\frac{4 a y}{\sqrt{ }\left(a^{2}-b^{2}\right)} \int \frac{\frac{1}{2} \sqrt{ }\left(\Sigma^{\prime}\right)}{s-\sigma^{\prime}} \frac{d s}{\sqrt{ } S}
$$

$$
=\frac{4 a y}{\sqrt{\left(a^{2}-b^{2}\right)}} E\left(f^{\prime} K\right)=\frac{4 a y}{\sqrt{\left(a^{2}-b^{2}\right)}} K \mathrm{zn} f^{\prime} K,
$$$$
4 a b x^{2} \int \frac{d \epsilon}{\left(a^{2}+\epsilon\right) \sqrt{ } E}=\frac{4 b x}{\sqrt{ }\left(a^{2}-b^{2}\right)} \int \frac{\frac{1}{2} V\left(-\Sigma^{\prime \prime}\right)}{s-\sigma^{\prime \prime}} \frac{d s}{\sqrt{ } S}
$$

$$
\begin{aligned}
& =\frac{4 b x}{\sqrt{\left(a^{2}-b^{2}\right)}} C\left(f^{\prime \prime} K^{\prime}\right) \\
& =\frac{4 b x}{\sqrt{\left(a^{2}-b^{2}\right)}}\left[K \mathrm{zs} f^{\prime \prime} K^{\prime}-\frac{1}{2} \pi\left(1-f^{\prime \prime}\right)\right] .
\end{aligned}
$$

Here, as in (3) § 2,

(12) $f=\int_{\sigma}^{s_{1}} \div \int_{\delta_{2}}^{s_{1}} \frac{d \sigma}{\sqrt{(-\Sigma)}}, \operatorname{sn}^{2} f K^{\prime}=\frac{\lambda}{\lambda-\mu}, \operatorname{cn}^{2} f K^{\prime}=\frac{-\mu}{\lambda-\mu}, \mathrm{dn}^{2} f K^{\prime}=\frac{-\nu}{\lambda-\nu}$,

(13) $f^{\prime}=\int_{s_{3}}^{\sigma^{\prime}} \div \int_{s_{3}}^{s_{2}} \frac{d s}{\sqrt{ } \bar{S}}, \operatorname{sn}^{2} f^{\prime} K=\frac{-b^{2}-\nu}{\mu-\nu}, \operatorname{cn}^{2} f^{\prime} K=\frac{b^{2}+\mu}{\mu-\nu}, \mathrm{dn}^{2} f^{\prime} K=\frac{b^{2}+\lambda}{\lambda-\nu}$,

(14) $f^{\prime \prime}=\int_{-\infty}^{\sigma^{\prime \prime}} \div \int_{-\infty}^{s_{3}} \frac{d \sigma}{\sqrt{(-\Sigma)}}, \operatorname{sn}^{2} f^{\prime \prime} K^{\prime}=\frac{\lambda-\nu}{a^{2}+\lambda}, \operatorname{cn}^{2} f^{\prime \prime} K^{\prime}=\frac{a^{2}+\nu}{a^{2}+\lambda}, \operatorname{dn}^{2} f^{\prime \prime} K^{\prime}=\frac{a^{2}+\mu}{a^{2}+\lambda}$. 
We have seen already in (5) and (15) $\S 34$, that

$$
\Omega=-\frac{d V}{d z}=4 A\left(f K^{\prime}\right)=2 \pi(1-f)-4 K \mathrm{zn} f K^{\prime},
$$

is the magnetic potential of the elliptic dise with uniform normal magnetization parallel to $O z$, or of the unit current round the elliptic ring; so also

$$
\Omega^{\prime}=-\frac{d V}{d y}=\frac{4 a}{\sqrt{\left(a^{2}-b^{2}\right)}} E\left(f^{\prime} K\right)=\frac{4 a}{\sqrt{\left(a^{2}-b^{2}\right)}} K \mathrm{zn} f^{\prime} K
$$

is the potential for uniform magnetization parallel to $O y$ the minor axis ; and

$$
\Omega^{\prime \prime}=-\frac{d V}{d x}=\frac{4 b}{\sqrt{\left(a^{2}-b^{2}\right)}} C\left(f^{\prime \prime} K^{\prime}\right)=\frac{4 b}{\sqrt{\left(a^{2}-b^{2}\right)}}\left[K \mathrm{zs} f^{\prime \prime} K^{\prime}-\frac{1}{2} \pi\left(1-f^{\prime \prime}\right)\right],
$$

for uniform magnetization parallel to $O x$ the major axis : and $\Omega^{\prime}, \Omega^{\prime \prime}$ are the components $F, G$ of the vector potential of the elliptic current.

For uniform magnetization of the elliptic disc with direction cosines $l, m, n$, the magnetic potential is

$$
n \Omega+m \Omega^{\prime}+l \Omega^{\prime \prime}
$$

Induced magnetism of a hollow ellipsoid of soft iron.

41. We may cite here the expression of the induction of a uniform magnetic field on a hollow ellipsoid of soft iron, bounded by confocals, of the family

$$
\frac{x^{2}}{a^{2}+\lambda}+\frac{y^{2}}{b^{2}+\lambda}+\frac{z^{2}}{\lambda}=1
$$

$\lambda_{0}$ and $\lambda_{1}$ defining the outer and inner surface of the shell.

We require the three incomplete II. E. I.'s

$$
\begin{gathered}
A, B, C=\int_{\lambda}^{\infty} \frac{d \epsilon}{\left(a^{2}+\epsilon, b^{2}+\epsilon, \epsilon\right) \vee P^{\prime}}, \\
P=4 \cdot a^{2}+\epsilon \cdot b^{2}+\epsilon \cdot \epsilon, \quad A+B+C=\frac{1}{\sqrt{ }\left(a^{2}+\lambda \cdot b^{2}+\lambda \cdot \lambda\right)} ;
\end{gathered}
$$

and with the fraction $h$ defined as in $\S 2$ by

$$
h=\int_{\lambda}^{\infty} \div \int_{0}^{\infty} \frac{d \epsilon}{\sqrt{ } P}
$$


we find from $\S 3$,

$$
\begin{gathered}
A=\frac{1}{a\left(a^{2}-b^{2}\right)}[h(K-H)-\mathrm{zn} h K], \\
B=\frac{a}{b^{2}\left(a^{2}-b^{2}\right)}\left[h\left(H-k^{2} K\right)-\mathrm{zn}(1-h) K\right], \\
C=\quad \frac{1}{a b^{2}} \quad[-h H+\mathrm{zs}(1-h) K], \\
k^{2}=1-\frac{b^{2}}{a^{2}}, \quad k^{\prime}=\frac{b}{a} .
\end{gathered}
$$

If the external inducing magnetic field has a potential

$$
V=X x+Y y+Z z,
$$

we can examine the components one at a time, so that if the component $X_{x}$ induces a magnetic potential $\Omega$ in the iron of the shell, $\Omega_{0}$ in the outside space, and $\Omega_{1}$ in the cavity, the magnetic conditions are satisfied by putiting

where

$$
\begin{gathered}
\Omega_{1}=L_{1} x, \quad \Omega_{0}=L_{0} \frac{A}{A_{0}} x, \\
\Omega=L_{0} \frac{A_{1}-A}{A_{1}-A_{0}} x+L_{1} \frac{A-A_{0}}{A_{1}-A_{0}} x,
\end{gathered}
$$

$$
\frac{A_{1}-A}{A_{1}-A_{0}}=\frac{\left(h_{1}-h\right)(K-H)-\left(\mathrm{zn} h_{1} K-\mathrm{zn} h K\right)}{\left(h_{1}-h_{0}\right)(K-H)-\left(\mathrm{zn} h_{1} K-\operatorname{zn} h_{0} K\right)}, \cdots ;
$$

and then determining $L_{0}$ and $L_{1}$ from the condition, with magnetic permeability $\mu$,

$$
\mu \frac{d}{d \nu}(X x+\Omega)=\frac{d}{d \nu_{1}}\left(X x+\Omega_{1}\right) \text { or } \frac{d}{d \nu_{0}}\left(X x+\Omega_{0}\right)
$$

in crossing the inner or outer surface, noticing that

$$
x \frac{d A}{d \nu}=-\frac{x}{a^{2}+\lambda} \frac{1}{\sqrt{ } P} \frac{d \lambda}{d \nu}=-\frac{2 p x}{a^{2}+\lambda} \frac{1}{\sqrt{ } P}=-\frac{2 l}{\sqrt{ } P} .
$$

Other cases for components of $V$ such as $x y, x y z, \ldots$ are given in the Journal de Physique (1881); the hydrodynamical analogues can be devel. oped by means of the same analysis. 
Potential of a circulcr disc, gravitational and magnetic.

42. For a circular disc put $a^{2}=b^{2}$,

$$
V=2 a^{2} \int_{\lambda}^{\infty} \cdot \sqrt{ }\left(1-\frac{x^{2}+y^{2}}{a^{2}+\epsilon}-\frac{z^{2}}{\epsilon}\right) \frac{d \epsilon}{\left(a^{2}+\epsilon\right) \sqrt{ } \epsilon} ;
$$

ellipsoidal coördinates are now unsuitable, and the elliptic coördinates, $v$ and $w$, must be employed, with the substitution

$$
\begin{gathered}
a^{2}+\epsilon=a^{2} \operatorname{ch}^{2} u, \quad \epsilon=a^{2} \operatorname{sh}^{2} u, \\
x^{2}+y^{2}=a^{2} \operatorname{ch}^{2} v \cos ^{2} w, \quad z^{2}=a^{2} \operatorname{sh}^{2} v \sin ^{2} w, \\
r_{1}, r_{2}=a(\operatorname{ch} v \pm \cos w),
\end{gathered}
$$

$r_{1}, r_{2}$ denoting the focal distances of $P$ from the foci $A, B$ in fig. $A$; and

$$
1-\frac{x^{2}+y^{2}}{a^{2}+\epsilon}-\frac{z^{2}}{\epsilon}=1-\frac{\operatorname{ch}^{2} v \cos ^{2} w}{\operatorname{ch}^{2} u}-\frac{\operatorname{sh}^{2} v \sin ^{2} v v}{\operatorname{sh}^{2} u}
$$

$$
=\frac{\left(\operatorname{ch}^{2} u-\operatorname{ch}^{2} v\right)\left(\operatorname{ch}^{2} u-\cos ^{2} w\right)}{\operatorname{ch}^{2} u \operatorname{sh}^{2} u},
$$

Next substitute

$$
\begin{gathered}
V=4 a \int_{v}^{\infty} \frac{V\left(\operatorname{ch}^{2} u-\operatorname{ch}^{2} v \cdot \operatorname{ch}^{2} u-\cos ^{2} w\right)}{\operatorname{ch}^{2} u \operatorname{sh} u} d u, \\
\Omega=-\frac{d V}{d z}=\int_{\lambda}^{\infty} \frac{2 a^{2} z d \epsilon}{\epsilon\left(a^{2}+\epsilon\right) \sqrt{ } \epsilon \sqrt{\left(1-\frac{x^{2}+y^{2}}{a^{2}+\epsilon}-\frac{z^{2}}{\epsilon}\right)}} \\
=4 \int_{v}^{\infty} \frac{\operatorname{sh} v \sin w d u}{\operatorname{sh} u V\left(\operatorname{ch}^{2} u-\operatorname{ch}^{2} v \cdot \operatorname{ch}^{2} u-\cos ^{2} w\right)} .
\end{gathered}
$$

$$
\begin{gathered}
\operatorname{sh}^{2} u=m^{2}(s-\sigma), \quad \operatorname{ch}^{2} u=m^{2}\left(s-s_{3}\right), \\
m^{2}\left(\sigma-s_{3}\right)=1, \quad \operatorname{ch}^{2} u=\frac{s-s_{3}}{\sigma-s_{3}}, \quad \operatorname{sh}^{2} u=\frac{s-\sigma}{\sigma-s_{3}}, \\
\operatorname{ch}^{2} u-\operatorname{ch}^{2} v=\frac{s-s_{1}}{\sigma-s_{3}}, \quad \operatorname{ch}^{2} u-\cos ^{2} w=\frac{s-s_{2}}{\sigma-s_{3}}, \\
\operatorname{ch}^{2} v=\frac{s_{1}-s_{3}}{\sigma-s_{3}}, \quad \operatorname{sh}^{2} v=\frac{s_{1}-\sigma}{\sigma-s_{3}}, \\
\cos ^{2} w=\frac{s_{2}-s_{3}}{\sigma-s_{3}}, \quad \sin ^{2} w=\frac{c-s_{2}}{\sigma-s_{3}}, \\
k^{2}=\frac{s_{2}-s_{3}}{s_{1}-s_{3}}=\frac{\cos ^{2} w}{\operatorname{ch}^{2} v}, \quad k=\frac{r_{1}-r_{2}}{r_{1}+r_{2}},
\end{gathered}
$$

as in MaXwelL's second expression for $M, \S 701$. 
The inequality sequence runs

$$
\infty>s>s_{1}>\sigma>s_{2}>s_{3}>-\infty,
$$

so that, from $\S 2$, dropping $K^{\prime}$,

$$
\operatorname{ch} v=\frac{1}{\operatorname{dn} f}=\frac{\operatorname{dn}(1-f)}{k}, \quad \cos w=\operatorname{dn}(1-f) .
$$

If then we write $A$ for $V\left(x^{2}+y^{2}\right), x=A \cos \phi, y=A \sin \phi$,

Since

$$
\begin{gathered}
\frac{A}{a}=\operatorname{ch} v \cos w=\frac{\operatorname{dn}(1-f)}{\operatorname{dn} f}=\frac{k}{\operatorname{dn}^{2} f}=\frac{\operatorname{dn}^{2}(1-f)}{k}, \\
\frac{z}{a}=\operatorname{sh} v \operatorname{sn} w=\frac{k^{\prime 2} \operatorname{sn} f \operatorname{cn} f}{\operatorname{dn}^{2} f}=\frac{k^{\prime 2}}{k} \operatorname{sn}(1-f) \operatorname{cn}(1-f), \\
\frac{r_{1}, r_{2}}{a}=\operatorname{ch} v \pm \cos w=\left(\frac{1}{k} \pm 1\right) \operatorname{dn}(1-f) .
\end{gathered}
$$

$$
\frac{\operatorname{sh} u d u}{\sqrt{ }\left(\operatorname{ch}^{2} u-\operatorname{ch}^{2} v \cdot \operatorname{ch}^{2} u-\cos ^{2} w\right)}=\frac{V\left(\sigma-s_{3}\right) d s}{\sqrt{ } S},
$$

equation (7), as before in $(15) \S 34$, becomes transformed to

$$
\Omega=4 \int_{s_{1}}^{\infty} \frac{\frac{1}{2} \sqrt{ }(-\Sigma)}{s-\sigma} \frac{d s}{\sqrt{S}}=4 A\left(f K^{\prime}\right)=2 \pi(1-f)-4 K \mathrm{zn} f K^{\prime}
$$

To make $s$ oscillate between $s_{2}$ and $s_{3}$, substitute

(21) $\quad \operatorname{ch}^{2} u=\frac{\sigma-s_{3}}{s-s_{3}}, \quad \operatorname{sh}^{2} u=\frac{\sigma-s}{s-s_{3}}, \quad \operatorname{ch}^{2} v=\frac{\sigma-s_{3}}{s_{2}-s_{3}}, \quad \cos ^{2} w=\frac{\sigma-s_{3}}{s_{2}-s_{3}}$.

43. The potential for uniform magnetization of the circular disc parallel to $A B$ is, as before in (16), (17), $\S 40$,

$$
\begin{aligned}
\Omega^{\prime} & =-\frac{d V}{d A}=\int_{\lambda}^{\infty} \frac{2 a^{2} A d \epsilon}{\left(a^{2}+\epsilon\right)^{2} V \epsilon \sqrt{\left(1-\frac{A^{2}}{a^{2}+\epsilon}-\frac{z^{2}}{\epsilon}\right)}} \\
& =\int_{v}^{\infty} \frac{4 \operatorname{ch} v \cos w \operatorname{sh} u \mathrm{dn}}{\operatorname{ch}^{2} u V\left(\operatorname{ch}^{2} u-\operatorname{ch}^{2} v \cdot \operatorname{ch}^{2} u-\cos ^{2} w\right)} \\
& =\frac{4}{\cos w} \int_{s_{1}}^{\infty} \frac{s_{2}-s_{3}}{s-s_{3}} \frac{V\left(s_{1}-s_{3}\right) d s}{\sqrt{ } S}=4 \frac{K-H}{\cos w} .
\end{aligned}
$$


From MaXwell's second expression in $\S 701$,

$$
M=4 \pi\left(r_{1}+r_{2}\right)(K-H)=8 \pi a \operatorname{ch} v(K-H)=2 \pi A \Omega^{\prime},
$$

and this is evident when we notice in figure $B$ that $M$ is composed of elements

$$
\frac{\cos \theta}{\bar{P} \bar{Q}^{\prime \prime}} a d \theta-\frac{\cos \theta}{P Q} a d \theta,
$$

which is the magnetic potential at $P$ of a strip $Q Q^{\prime \prime}$ parallel to $A B$, magnetized longitudinally; and these elements are integrated over the circle $A Q B$, and multiplied afterwards by $2 \pi A$ to obtain $M$ for the two circles.

Replace $A$ by $x$, then at any point $(x, 0, z)$

$$
\begin{gathered}
M=2 \pi x \Omega^{\prime}=-2 \pi x \frac{d V}{d x}, \quad 2 \pi \Omega=-2 \pi \frac{d V}{d z}, \\
2 \pi \frac{d \Omega}{d x}=-2 \pi \frac{d^{2} V}{d x d z}=\frac{1}{x} \frac{d M}{d z}, \\
2 \pi \frac{d \Omega}{d z}=-2 \pi \frac{d^{2} V}{d z^{2}}=2 \pi\left(\frac{d^{2} V}{d x^{2}}+\frac{1}{x} \frac{d V}{d x}\right)=-\frac{1}{x} \frac{d M}{d x},
\end{gathered}
$$

and a magnetic line of force along which $M$ is constant is the orthogonal trajectory of the equipotential surfaces $\Omega=$ constant; in fact $M$ is the Stokes function of the magnetic potential, and

or as they may be written

$$
\begin{aligned}
& \frac{d^{2} \Omega}{d z^{2}}+\frac{d^{2} \Omega}{d x^{2}}+\frac{1}{x} \frac{d \Omega}{d x}=0 \\
& \frac{d^{2} M}{d z^{2}}+\frac{d^{2} M}{d x^{2}}-\frac{1}{x} \frac{d M}{d x}=0
\end{aligned}
$$

$$
\begin{aligned}
& \frac{d}{d z}\left(x \frac{d \Omega}{d z}\right)+\frac{d}{d x}\left(x \frac{d \Omega}{d x}\right)=0 \\
& \frac{d}{d z}\left(\frac{1}{x} \frac{d M}{d z}\right)+\frac{d}{d x}\left(\frac{1}{x} \frac{d M}{d x}\right)=0
\end{aligned}
$$

These are MAXwELL's results of $\S 703$, obtained from independent physical considerations when $x$ and $z$ are replaced by his $a$ and $b$; the symmetry round the axis $z$ shows that the integration employed in MAxweLl's treatment can be effected, so that his result, where $V$ and $\Omega$ are equivalent, may be written

$$
\begin{aligned}
& \frac{d M}{d a}=\int_{0}^{2 \pi} a \frac{d V}{d y} d \theta=2 \pi a \frac{d V}{d y}, \\
& \frac{d M}{d b}=-\int a \frac{d V}{d r} d \theta=-2 \pi a \frac{d V}{d r} .
\end{aligned}
$$


The potential of the circular disc is now written, either from (1) or (6), § 42,

$$
\begin{aligned}
V= & 4 a \int_{0}^{\infty}\left(1-\frac{\operatorname{ch}^{2} v \cos ^{2} w}{\operatorname{ch}^{2} u}-\frac{\operatorname{sh}^{2} v \sin ^{2} w}{\operatorname{sh}^{2} u}\right) \frac{\operatorname{sh} u d u}{V\left[\left(\operatorname{ch}^{2} u-\operatorname{ch}^{2} v\right) \cdot\left(\operatorname{ch} u-\cos ^{2} w\right)\right]} \\
& =4 \int_{s_{1}}^{\infty}\left[a-A \frac{V\left(s_{1}-s_{3} \cdot s_{2}-s_{3}\right)}{s-s_{3}}-z \frac{V\left(s_{1}-\sigma \cdot \sigma-s_{2}\right)}{s-\sigma}\right] \frac{V\left(\sigma-s_{3}\right) d s}{V S} \\
& =4 a \sqrt{ }\left(\frac{\sigma-s_{3}}{s_{1}-s_{3}}\right) K-4 A \sqrt{ }\left(\frac{\sigma-s_{3}}{s_{2}-s_{3}}\right)(K-H)-4 z A\left(f K^{\prime}\right) \\
& =\frac{4 a K}{\operatorname{ch} v}-A \Omega^{\prime}-z \Omega,
\end{aligned}
$$

of which each term can be interpreted by means of the homogeneity relation

$$
V=a \frac{d V}{d a}+A \frac{d V}{d A}+z \frac{d V}{d z}
$$

since

$$
\frac{d V}{d z}=-\Omega, \quad \frac{d V}{d A}=-\Omega^{\prime},
$$

and therefore

$$
\frac{d V}{d a}=\frac{4 K}{\operatorname{ch} v}=\frac{8 a K}{r_{1}+r_{2}},
$$

the potential at $\boldsymbol{P}$ of the circular ring round the rim of the disc.

Similarly in the case of the elliptic dise in $\S 40$ the term in (8)

$$
4 a b \int \frac{d \epsilon}{\sqrt{ } \bar{E}}=\frac{4 a b K}{\sqrt{ }(\lambda-\nu)}
$$

may be interpreted as the potential at $P$ of a ring round the ellipse cut off by a consecutive confocal ellipse, so that the thickness is inversely proportional to the perpendicular from the center on the tangent.

The Stokes function of a circular plate and ring.

44. Denote the Stokes function of the plate by $N$, using $b$ again instead of $z$; then, as in $\S \S 31,43$,

$$
\begin{aligned}
\frac{d N}{d b}=2 \pi A \frac{d V}{d A} & =-2 \pi A \Omega^{\prime}=-4 \pi\left(r_{1}+r_{2}\right)(K-H), \\
\frac{d N}{d A} & =-2 \pi A \frac{d V}{d b}=2 \pi A \Omega \\
& =2 \pi A\left[2 \pi(1-f)-4 K \operatorname{mn} f K^{\prime}\right] \\
& =2 \pi A \cdot 4 A\left(f K^{\prime}\right),
\end{aligned}
$$


and by analogy

$$
\frac{d N}{d a}=2 \pi a \cdot 4 A(1-f) K^{\prime}=2 \pi a\left[2 \pi f-4 K \mathrm{zn}(1-f) K^{\prime}\right],
$$

a change of $f$ into $1-f$, and $A$ into $a$; and this term is the Stokes function of the circular ring, of which the potential function is given in (1), $\S 33$ : so that the Stokes function at $P$ of the circle $A Q B$ is $2 \pi a$ times the apparent area at $P^{\prime}$.

Then by the homogeneity of the second degree in $a, A, b$, of $N$

$$
\begin{gathered}
2 N=a \frac{d N}{d a}+A \frac{d N}{d A}+b \frac{d N}{d b} \\
=8 \pi a^{2} A(1-f) K^{\prime}+8 \pi A^{2} \cdot A f K^{\prime}-4 \pi b\left(r_{1}+r_{2}\right)(K-H), \\
N=4 \pi\left(a^{2}-A^{2}\right) B\left(f K^{\prime}\right)+2 \pi^{2} A^{2}-2 \pi b\left(r_{1}+r_{2}\right)\left(K-H+K \mathrm{dn}^{2} f K^{\prime}\right) .
\end{gathered}
$$

For points on the plate, $f=0, N=2 \pi^{2} A^{2}$ : while $f=1$ for points in its plane outside, and $N=2 \pi^{2} a^{2}$; and $N-2 \pi^{2} A^{2}$ may be taken as the Stokes function at $P$ of this plate and a coaxial equal and opposite plate at the level of $P$.

Changing the superficial density from unity to $1 / 2 \pi p$, we shall see in the sequel that

$$
\frac{2 \pi^{2} A^{2}-N}{2 \pi p}=M,
$$

where $M$ is the function in $\S 54$ investigated by Viriamu Jones for a helical current, or the equivalent cylindrical current sheet; and the potential of the two end plates is the magnetic potential of the cylinder magnetized longitudinally, while the Stokes function $N-2 \pi^{2} A^{2}$ is the magnetic potential for magnetization across the axis of the cylinder.

\section{The various dissections of an integral.}

45. To arrive geometrically at the expression of the conical angle $\Omega$ by means of the angles $\theta, \phi, \psi, \chi, \omega$ defined previously on fig. $A$ and $B$, we employ the idea given by MAXwELL in E. and M. $\S 418$, that the area $\Omega$, cut out on unit sphere by a cone whose vertex is at the centre, is equal to $2 \pi$ minus the length $\Phi$ of the curve traced out on the sphere by the reciprocal cone; this is a direct result and generalisation of the theorem that the sum of the area of a spherical triangle and the perimeter of the polar triangle is $2 \pi$.

The same idea is employed by Schwarz in his memoir Die Scheinbare Grösse des Ellipsoides, the apparent area and conical angle being the same thing, either of the ellipsoid, or of the tangent cone, or any elliptic section of it.

If the tangent plane $P Q Y$ of the cone, shown in elevation and plan in figures $A$ and $B$, turns through an angle $d \Phi$ while $O Q$ turns through the small angle 
$d \theta$, and if the normal line $P q$ of the cone cuts the plane $A Q B$ in $q$ on $Y M$ produced (fig. $B$ ), then $Q$ and $q$ describe curves which are polar reciprocal with respect to $M$; so that $Q M y$ is perpendicular to $q y$ the tangent at $q$; and the sector velocity of $q$ round $M$

$$
\begin{gathered}
\frac{1}{2} M q^{2} \frac{d \theta}{d t}=\frac{1}{2} P q^{2} \frac{d \Phi}{d t} \cos M y P, \\
\frac{d \Phi}{d \theta}=\frac{M q^{2}}{P q^{2}} \frac{P y}{M y}=\frac{P M^{2}}{P \bar{Y}^{2}} \cdot \frac{P Q}{P M}=\frac{1}{1-\frac{Q Y^{2}}{P Q^{2}}} \frac{P M}{P Q},
\end{gathered}
$$

so that, in MAXweLL's notation, with $O M=A$,

Now from $\S 27$

$$
2 \pi-\Omega=\Phi=\int_{0}^{2 \pi} \frac{1}{1-\frac{A^{2} \sin ^{2} \theta}{P Q^{2}}} \cdot \frac{b d \theta}{P Q} .
$$

$$
\frac{A^{2} \sin ^{2} \theta}{P Q^{2}}=\frac{4 a^{2} A^{2} \sin ^{2} \theta}{\left(r_{1}+r_{2}\right)^{2} C Q^{2}}=\left(\frac{2 A}{r_{1}+r_{2}}\right)^{2} \sin ^{2} \chi, \quad \frac{b d \theta}{P Q}=\frac{2 b}{r_{1}+r_{2}} \frac{d \chi}{\Delta \chi},
$$

so that, in LEGENDRE's form of $\S 14$,

$$
\Phi=\int_{0}^{\frac{1}{2} \pi} \frac{4 \sqrt{ } \alpha}{1+n \sin ^{2} \chi} \frac{d \chi}{\Delta \chi},
$$

(6) $\quad n=-\left(\frac{2 A}{r_{1}+r_{2}}\right)^{2}, \quad k=\frac{r_{1}-r_{2}}{r_{1}+r_{2}}, \quad \alpha=(1+n)\left(1+\frac{k^{2}}{n}\right)=\left(\frac{2 b}{r_{1}+r_{2}}\right)^{2}$.

To reduce $\Phi$ to our form $B$ in $\S 5$, we put

$$
\sin ^{2} \chi=\frac{s-s_{3}}{s_{2}-s_{3}}, \quad \frac{d \chi}{\Delta \chi}=\frac{V\left(s_{1}-s_{3}\right) d s}{\sqrt{ } S},
$$

and interpreted in fig. $A$, where $O M=A, O C=a k, O C^{\prime}=a / k$,

$$
\begin{gathered}
\frac{\sigma-s_{3}}{s_{2}-s_{3}}=-\frac{1}{n}=\left(\frac{r_{1}+r_{2}}{2 A}\right)^{2}, \frac{\sigma-s_{3}}{s_{1}-s_{3}}=\operatorname{dn}^{2} f K^{\prime}=\left(\frac{r_{1}-r_{2}}{2 A}\right)^{2}=k \frac{r_{1}^{2}-r_{2}^{2}}{4 A^{2}}=k \frac{a}{A}=\frac{O C}{O M}, \\
\frac{\sigma-s_{2}}{s_{2}-s_{3}}=\frac{\left(r_{1}+r\right)^{2}-4 A^{2}}{4 A^{2}}, \quad \frac{s_{1}-\sigma}{s_{1}-s_{3}}=\frac{4 A^{2}-\left(r_{1}-r_{2}\right)^{2}}{4 A^{2}} \\
\frac{s_{1}-\sigma \cdot \sigma-s_{2}}{s_{1}-s_{3} \cdot s_{2}-s_{3}}=\frac{b^{2}}{A^{2}}
\end{gathered}
$$

so that, as before in $\S 34$,

$$
\begin{gathered}
\Phi=4 \int_{s_{3}}^{s_{2} \frac{1}{2} V(-\Sigma)} \frac{d s}{\sigma-s} \frac{d s}{\sqrt{ }}=4 B\left(f K^{\prime}\right), \\
\Omega=2 \pi-4 B\left(f K^{\prime}\right)=4 A\left(f K^{\prime}\right)=4 \int_{s_{1}}^{\infty} \frac{\left.\frac{1}{2} \sqrt{ } \sqrt{ }-\Sigma\right)}{s-\sigma} \frac{d s}{\sqrt{ } S^{\prime}}
\end{gathered}
$$


46. If $d \alpha$ denotes an element of area at any point $Q$ inside the circle, due to any mode of dissection

$$
V=\int \frac{d \alpha}{P} \bar{Q}, \quad \Omega=\int \frac{b d \alpha}{P Q^{3}},
$$

and the integrals which arise in the determination of these potential functions change in appearance with the method of dissection employed by the use of each system of variables; so it will save repetition in the sequel if we cite here some preliminary lemmas.

I. The potential at a point $F$ on its axis of a flat ring-shaped disc, bounded internally and externally by concentric circles on diameter $a b$ and $A B$, is

$$
\frac{\text { area }}{\frac{1}{2}(F A+F a)}=2 \pi(F A-F a),
$$

and thence also the same for any fraction of the ring cut out by two radii at an angle $\theta$, replacing $2 \pi$.

II. The potential at $P$ of the line $A B$ in fig. $A$ is

(3) $\int_{-a}^{a} \frac{d x}{P} \bar{Q}=\log \frac{P A+P B+A B}{P A+P B-A B}=2 t^{-1} \frac{A B}{P A+P B}=2 t^{-1} \frac{P A-P B}{2 O M}$, and when $P$ is at $F$, the potential of $A B$ is twice the potential of $O A$, and

$$
2 \operatorname{th}^{-1} \frac{O A}{F A} \text {. }
$$

In the elliptic coördinates of $\S 42$ the potential at $P$ assumes the various forms

$$
\begin{aligned}
\log \frac{\operatorname{ch} v+1}{\operatorname{ch} v-1} & =2 \log \frac{\operatorname{ch} v+1}{\operatorname{sh} v}=2 \log \frac{\operatorname{sh} v}{\operatorname{ch} v-1}=\int_{v}^{\infty} \frac{d v}{\operatorname{sh} v} \\
& =2 \operatorname{sh}^{-1} \frac{1}{\operatorname{sh} v}=2 \operatorname{ch}^{-1} \frac{\operatorname{ch} v}{\operatorname{sh} v}=2 \operatorname{th}^{-1} \frac{1}{\operatorname{ch} v}=\operatorname{ch}^{-1} \frac{\operatorname{ch}^{2} v+1}{\operatorname{ch}^{2} v-1}, \text { etc. }
\end{aligned}
$$

The Stokes function at $P$ of the line $A B$ is

$$
2 \pi(P A-P B)=4 \pi a \cos w,
$$

an expression much simpler.

III. The conical angle of the ring as seen from $F$ on its axis is

$$
2 \pi\left(\frac{F O}{F a}-\frac{F O}{F A}\right)=\frac{F O \times \text { area }}{F A \cdot F^{\prime} a \cdot \frac{1}{2}(F A+F a)},
$$

and when the diameters $a b, A B$ are nearly equal, this may be replaced by

$$
\frac{F O \times \text { area }}{F A^{3}}
$$


So also for any strip cut out by two radii ; and if the radii make a small angle $d \theta$, the same expression holds when the plane of the strip is tilted about its mean radius $O A$ through any angle, provided $F O$ is replaced by the perpendicular $F M$ on the plane, and the expression becomes

$$
\left(\frac{F M}{\bar{F} a}-\frac{F M}{F A}\right) d \theta \text {. }
$$

IV. For a narrow parallel strip such as $Q Q^{\prime \prime}$, of breadth $d y$, and $P R$ the perpendicular on it from $P$, the conical angle at $P$ is

$$
\frac{P M}{P R^{2}}\left(\cos P Q R-\cos P Q^{\prime \prime} R\right) d y=\frac{P M \times \text { area }}{P R^{2} \cdot Q Q^{\prime \prime}}\left(\frac{R Q}{P} \frac{R}{Q}-\frac{R Q^{\prime \prime}}{P Q^{\prime \prime}}\right) .
$$

47. Considering that $V+b \Omega$ is composed of complete I. and II. E. I.'s, the complete III. E. I. which arises in the determination of $\Omega$ will serve at the same time for $V$; and according to the method of dissection we shall find it depend on one of the five following forms, which we shall represent in the following notation, in which the III. E. I. is not restricted to be complete;

$$
\begin{aligned}
& \Omega(M Q)=\int_{0}^{\theta} \frac{a A \cos \theta+a^{2}}{M Q^{2}} \frac{b d \theta}{P Q}=\int \frac{a \cdot M Y}{M Q^{2}} \frac{b d \theta}{P Q}, \\
& \Omega(P Y)=\int \frac{P Q^{2} b d \theta}{P Y^{2}-P Q}, \\
& \Omega(P Z)=\int \frac{a A \cos \theta+A^{2}+b^{2}}{P Z^{2}} \frac{b d \theta}{P Q},
\end{aligned}
$$

where $P Z$ is the perpendicular on $O Q$, and $P Z^{2}=A^{2} \sin ^{2} \theta+b^{2}$;

$$
\begin{aligned}
& \Omega(P N)=\int \frac{Q N^{2}}{P N^{2}} \frac{b d \theta}{P Q}, \\
& \Omega(P R)=\int \frac{a \cos \theta(a \cos \theta+A)}{P R^{2}} \frac{b d \theta}{P Q}=\int \frac{O N \cdot M N}{P R^{2}} \frac{b d \theta}{P} \bar{Q},
\end{aligned}
$$

and these III. E. I.'s are connected in pairs by the ten equations following, as is verified by differentiation :

$$
\begin{aligned}
\Omega(P Y)-\Omega(P Z) & =I_{1}=\sin ^{-1} \frac{M Z \cdot Q Z}{P Y \cdot P Z}=\cos ^{-1} \frac{P M \cdot P Q}{P Y \cdot P} \\
& =\frac{1}{2} \pi-\text { angle between planes } P Q Y, P Q Z \\
\Omega(M Q)-\Omega(P Z) & =I_{2}=\sin ^{-1} \frac{M Z \cdot P Q}{P Z \cdot M Q}=\cos ^{-1} \frac{M N \cdot M P}{P Z \cdot M Q} \\
& =\text { angle between } M P Q, Q P Z
\end{aligned}
$$




$$
\begin{aligned}
\Omega(M Q)-\Omega(P Y) & =I_{3}=\sin ^{-1} \frac{M Z \cdot M P}{P Y \cdot M Q}=\cos ^{-1} \frac{Q Z \cdot P Q}{P Y \cdot M Q} \\
& =\text { angle between } P Q T, P Q Y
\end{aligned}
$$

$$
\begin{aligned}
\Omega(M Q)+\Omega(P N) & =I_{4}=\sin ^{-1} \frac{Q N \cdot P M}{P N \cdot M Q}=\cos ^{-1} \frac{M N \cdot P Q}{\overline{P N} \cdot M Q} \\
& =\text { angle between } P Q T, Q M R
\end{aligned}
$$

$$
\begin{aligned}
\Omega(M Q)+\Omega(P R) & =I_{s}=\sin ^{-1} \frac{Q N \cdot P Q}{P R \cdot M Q}=\cos ^{-1} \frac{M N \cdot P M}{P R \cdot M Q} \\
& =\text { angle between } M P Q, M Q R
\end{aligned}
$$

$$
\begin{aligned}
\Omega(P R)-\Omega(P N) & =I_{6}=\sin ^{-1} \frac{Q N \cdot M N}{P R \cdot P N}=\cos ^{-1} \frac{P M \cdot P Q}{P R \cdot P N} \\
& =\frac{1}{2} \pi-\text { angle between } Q P R, Q P N
\end{aligned}
$$

$$
\Omega(P R)+\Omega(P Y)=I_{7}=\sin ^{-1}=\frac{\sin \theta\left(a A \cos \theta+a^{2}+b^{2}\right)}{P R \cdot P Y^{2}}
$$

$$
\begin{aligned}
& =\cos ^{-1} \frac{P M \cos \theta \cdot P Q}{P R \cdot P Y} \\
& =\frac{1}{2} \pi-\text { angle between } Q P R, Q P Y \text {; } \\
& \Omega(P R)+\Omega(P Z)=I_{8}=\sin ^{-1} \frac{P M \sin \theta \cdot P Q}{P R \cdot P Z}=\cos ^{-1} \frac{a A \sin ^{2} \theta-b^{2} \cos \theta}{P R \cdot P Z} \\
& \text { = angle between } Q P Z, Q P R \text {; } \\
& \Omega(P Y)-\Omega(P N)=I_{9}=\sin ^{-1} \frac{P M \sin \theta \cdot P Q}{P Y \cdot P N} \\
& =\cos ^{-1} \frac{\sin \theta\left(a A \cos \theta+A^{2}+b^{2}\right)}{P Y \cdot P N} \\
& \text { = angle between } Q P N, Q P Y \text {; } \\
& \Omega(P Z)+\Omega(P N)=I_{10}=\sin ^{-1} \frac{\sin \theta\left(a A \cos \theta+A^{2}+b^{2}\right)}{P Z \cdot P N} \\
& =\cos ^{-1} \frac{P M \cos \theta \cdot P Q}{P Z \cdot P N} \\
& =\frac{1}{2} \pi \text { - angle between } Q P N, Q P Z \text {. }
\end{aligned}
$$


In a complete III. E. I., when $\theta$ ranges from 0 to $2 \pi$, the $I$ term disappears or is $2 \pi$, and

$$
\begin{aligned}
\Phi & =2 \pi-\Omega=\Omega(M Q)=\Omega(P Y)=\Omega(P Z) \\
& =2 \pi-\Omega(P N)=2 \pi-\Omega(P R) .
\end{aligned}
$$

We shall require in conjunction with $\Omega(P Z)$ the associated integral

and then

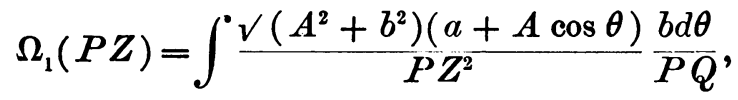

$$
\begin{aligned}
& \Omega_{1}(P Z)+\Omega(P Z)=\int \frac{a+\sqrt{ }\left(A^{2}+b^{2}\right)}{1\left(A^{2}+b^{2}\right)-A \cos \theta} \frac{b d \theta}{P}, \\
& \Omega_{1}(P Z)-\Omega(P Z)=\int \frac{a-\sqrt{ }\left(A^{2}+b^{2}\right)}{\sqrt{\left(A^{2}+b^{2}\right)+A \cos \theta} \frac{b d \theta}{P Q},}
\end{aligned}
$$

two III. E. I.'s, with parameter $f_{1} F^{\prime}, f_{2} F^{\prime}$, such that

$$
\operatorname{sn} f_{1} F^{\prime}=\frac{a-V\left(A^{2}+b^{2}\right)}{r_{2}}, \quad \text { sn } f_{2} F^{\prime}=\frac{r_{1}}{a+\sqrt{ }\left(A^{2}+b^{2}\right)},
$$

leading to a simple geometrical construction; and it is found that $2 \pi-\Omega_{1}(P Z)$ is the conical angle subtended by the circle at $P_{1}$ on $O P$ produced.

So too in conjunction with $\Omega(P R)$ we require

$$
\begin{gathered}
\Omega_{1}(P Q)=\int \frac{V\left(a^{2}+b^{2}\right)(A+a \cos \theta)}{P R^{2}} \frac{b d \theta}{P Q}, \\
\Omega_{1}(P R)+\Omega(P R)=\int \frac{A+a \cos \theta}{\sqrt{ }\left(a^{2}+b^{2}\right)-a \cos \theta} \frac{b d \theta}{P Q}, \\
\Omega_{1}(P R)-\Omega(P R)=\int \frac{A+a \cos \theta}{\sqrt{\left(a^{2}+b^{2}\right)+a \cos \theta} \frac{b d \theta}{P Q},}
\end{gathered}
$$

and $\Omega_{1}(P R)$ is the conical angle at $P_{2}$ where $P_{1} P_{2}$ passes through $B$, or $P_{2} P^{\prime}$ is parallel to $A B$.

48. Thus with sector elements $\frac{1}{2} a^{2} d \theta$ for the determination of $V$, the potential $d V$ at $P$ of the element about $O Q$ is given by

$$
\begin{aligned}
\frac{d V}{d \theta} & =\int_{0}^{a} \frac{r d r}{P Q}=\int \frac{r+A \cos \theta}{P Q} d \theta-\int \frac{A \cos \theta d \theta}{P Q} \\
& =P Q-P O-2 A \cos \theta \mathrm{th}^{-1} \frac{a}{P Q+P O},
\end{aligned}
$$

of which the first part is by Lemma I the potential of the strip $O Q q^{\prime} o$, between $O Q, o q^{\prime}$ radiating from $Z$, and the second is by Lemma II the potential of a shaving of uniform breadth $d \theta \cdot A \cos \theta$ and length $O Q$. 
As required in integration by parts, the second part of (1) can be written

(3)

$$
\begin{gathered}
2 \frac{d}{d \theta}\left(A \sin \theta \operatorname{th}^{-1} \frac{a}{P Q+P O}\right)-\frac{a^{2} A^{2} \sin ^{2} \theta}{P Q \cdot P O+a A \cos \theta+A^{2}+b^{2}} \frac{1}{P Q} \\
=2 \frac{d}{d \theta}\left(A \sin \theta \mathrm{th}^{-1} \frac{a}{P Q+P O}\right)-\frac{P Q \cdot P O-a A \cos \theta-A^{2}-b^{2}}{P Z^{2}} \frac{A^{2} \sin ^{2} \theta}{P Q} \\
=2 \frac{d}{d \theta}\left(A \sin \theta \mathrm{th}^{-1} \frac{a}{P Q+P O}\right)-P O\left(1-b^{2}\right) \\
+\frac{a A \cos \theta+A^{2}+b^{2}}{P Q}-\frac{b d \Omega(P Z)}{d \theta} ;
\end{gathered}
$$

So also, if $d \Omega$ is the conical angle subtended by the sector element about $O Q$, the application of Lemmas III and IV shows that

$$
\begin{aligned}
\frac{d \Omega}{d \theta} & =\frac{P M}{P \bar{O}}-\frac{P M}{P Q}-\frac{P M}{P Z^{2}}\left(\frac{Q Z}{P Q}-\frac{O Z}{P O}\right) A \cos \theta \\
& =b \frac{P O}{P Z^{2}}-\frac{d \Omega(P Z)}{d \theta}=b \frac{P O}{P} \frac{Z^{2}}{d \theta}+\frac{d I_{1}}{d \theta}-\frac{d \Omega(P Y)}{d \theta},
\end{aligned}
$$

so that

$$
\frac{d V}{d \theta}+b \frac{d \Omega}{d \theta}=\frac{a^{2}+a A \cos \theta}{P Q}-2 \frac{d}{d \theta}\left(A \sin \theta \operatorname{th}^{-1} \frac{a}{P Q+P O}\right)
$$

and thence the previous expressions in (13), § 43.

49. The dissection which leads directly to Mrnchis's integral in the Philosophical Magazine, February, 1894, is made by lines radiating from $M$.

Denoting the angle $A M Q$ by $\eta$,

(1) $\frac{1}{2} M Q^{2} d \eta=$ sector element about $M Q=\frac{1}{2} M Y \cdot a d \theta=\frac{1}{2}\left(a^{2}+a A \cos \theta\right) d \theta$, and by Lemma III,

$$
\begin{gathered}
d \Omega=\left(1-\frac{P M}{\overline{P Q}}\right) d \eta \\
\frac{d \Omega}{d \theta}=\frac{d \eta}{d \theta}-\frac{a^{2}+a A \cos \theta}{M Q^{2}} \frac{b}{P Q}=\frac{d \eta}{d \theta}-\frac{d \Omega(M Q)}{d \theta},
\end{gathered}
$$

so that for any incomplete arc $A Q$

$$
\Omega=\eta-\Omega(M Q) .
$$

Now with the substitution of $\$ 26$ and in addition

so that

(6) $b^{2}=m^{2}\left(t_{1}-\tau\right), \quad(a \sim A)^{2}=m^{2}\left(\tau-t_{2}\right), \quad(a+A)^{2}=m^{2}\left(\tau-t_{3}\right)$, 
then when $M$ is inside the circle

$$
\begin{gathered}
a-A=m \vee\left(\tau-t_{2}\right), \quad a=\frac{1}{2} n\left[\left(\tau-t_{2}\right)+v\left(\tau-t_{3}\right)\right], \\
a A \cos \theta=\frac{1}{4} m^{2}\left(t_{2}+t_{3}-2 t\right), \\
a A \cos \theta+a^{2}=\frac{1}{2} m^{2}\left[\tau-t+V\left(\tau-t_{2} \cdot \tau-t_{3}\right)\right] .
\end{gathered}
$$

Integrating round the circle, the angle $\eta$ makes a complete revolution when $M$ is inside, and

$$
\begin{aligned}
& \Omega=2 \pi-\int_{t_{3}}^{t_{2}}\left[1+\frac{V\left(\tau-t_{2} \cdot \tau-t_{3}\right)}{\tau-t}\right] \frac{V\left(t_{1}-\tau\right) d t}{\frac{1}{2} \sqrt{ } T} \\
& =2 \pi-2 \sqrt{\frac{t_{1}-\tau}{t_{1}-t_{3}}} \int \frac{V\left(t_{1}-t_{3}\right) d t}{\sqrt{ }}-2 \int \frac{\frac{1}{2} V\left(-T^{\prime}\right)}{\tau-t} \frac{d t}{\sqrt{T}} \\
& =2 \pi-2 F c^{\prime} \text { sn } 2 f F^{\prime}-2 B\left(2 f F^{\prime}\right) \\
& =2 \pi(1-f)-2 F \mathrm{zn} 2 f F^{\prime}-2 F c^{\prime} \text { sn } 2 f F^{\prime} ;
\end{aligned}
$$

hence Minchin's result obtained directly.

If $M$ is outside the circle, the angle $\eta$ oscillates; but we must take

and now

$$
a=\frac{1}{2} m\left[\sqrt{ }\left(\tau-t_{3}\right)-V\left(\tau-t_{2}\right)\right],
$$

$$
\Omega=2 \int\left[\frac{\sqrt{ }\left(\tau-t_{2} \cdot \tau-t_{3}\right)}{\tau-t}-1\right] \frac{d t}{\sqrt{ } T}=2 \pi f+2 F_{\mathrm{zn}} 2 f F^{\prime}-2 F c^{\prime} \operatorname{sn} f F^{\prime},
$$

equivalent to a change of $f$ into $1-f$, as in going from $P$ to $P^{\prime \prime}$ so that (10) may be taken to hold for all cases.

As $P$ travels round the circle $C P C^{\prime}$ the quantities $c, k, F, E, K, H$ do not vary, and $f$ may be taken as the independent variable.

Starting from $C$ in the plane of the disc, where $f=0$,

$$
\Omega=2 \pi, \quad V=4 a \frac{E+c^{\prime} F}{1+c^{\prime}}=4 a H, \quad \frac{V}{A C}=2\left(E+c^{\prime} F^{\prime}\right) .
$$

At $C^{\prime}$ outside the dise in its plane, $f=1$,

(14) $\Omega=0$,

$$
V=4 a \frac{E-c^{\prime} F}{1-c^{\prime}}=4 a \frac{H-k^{\prime 2} K}{k}, \quad \frac{V}{A C^{\prime}}=2\left(E-c^{\prime} F^{\prime}\right) .
$$

As $P$ travels to $P^{\prime \prime}, f$ increases from $f$ to $f^{\prime \prime}=1-f$, so that

$$
\Omega^{\prime \prime}=2 \pi f+2 F \text { zn } 2 f F^{\prime}-2 F c^{\prime} \text { sn } 2 f F^{\prime},
$$

$$
\Omega+\Omega^{\prime \prime}=2 \pi-4 F c^{\prime} \operatorname{sn} 2 f F^{\prime}=2 \pi-4 F \sin \psi^{\prime}, \quad \Omega-\Omega^{\prime \prime}=2 \pi-4 B\left(2 f F^{\prime}\right),
$$

and $\Omega^{\prime \prime}$ is the apparent area of the coaxial circle through $P$ seen from any point on the circle $A B$. 


$$
\frac{V}{A P}+\frac{V^{\prime \prime}}{A P^{\prime \prime}}=4 E-4 F \sin ^{2} \psi^{\prime}-\left(\Omega+\Omega^{\prime \prime}\right) \sin \psi^{\prime}=4 E-2 \pi \sin \psi^{\prime},
$$

which is Mrnchis's equation (23), p. 212, Philosophical Magazine, February, 1894.

When $P$ reaches $P^{\prime}, f$ has increased to $f^{\prime}=1+f$,

$$
\begin{gathered}
\Omega^{\prime}=-2 \pi f+2 F^{\prime} \text { zn } 2 f F^{\prime}+2 F \sin \psi^{\prime}, \\
\Omega-\Omega^{\prime}=2 \pi,
\end{gathered}
$$

but

$$
V^{\prime}-V^{\prime \prime}=0 .
$$

At $P^{\prime \prime \prime}, f$ has reached $f^{\prime \prime \prime}=2-f$;

$$
\Omega-\Omega^{\prime \prime \prime}=4 \pi, \quad V-V^{\prime \prime \prime}=0,
$$

so that $V$ has no cyclic constant as $P$ describes a curve linked with the circular edge; a cylic constant like the $4 \pi$ for $\Omega$ would imply a non-conservative potential and the possibility of the creation of energy, called otherwise Perpetual Motion.

50. Dissected by $Q N$ into strips $y d x=a \sin ^{2} \theta d \theta$, then by Lemma IV, $\S \mathbf{4 6}$,

$$
d \Omega=\frac{b d x}{P \bar{N}^{2}} \frac{a \sin \theta}{P Q}=\frac{a^{2} \sin ^{2} \theta}{P N^{2}} \frac{b d \theta}{P Q}, \quad \frac{d \Omega}{d \theta}=\frac{d \Omega(P N)}{d \theta},
$$

so that $\chi$ of $\S 27$ is the appropriate coördinate angle, leading to E. F.'s of $h K$ and $f K^{\prime}$, and

$$
\begin{aligned}
\Omega & =\int_{0}^{2 \pi} \frac{a^{2} \sin ^{2} \theta}{\left(\frac{r_{1}+r_{2}}{2 a}\right)^{2} C Q^{2}-a^{2} \sin ^{2} \theta} \frac{b d \theta}{P} Q \\
& =\int^{\frac{1}{2 \pi}} \frac{4 \sin ^{2} \chi}{\left(\frac{r_{1}+r_{2}}{2 a}\right)^{2}-\sin ^{2} \chi} \frac{b}{r_{1}+r_{2}} \frac{d \chi}{\Delta \chi},
\end{aligned}
$$

which is reduced to the form $A$ of $\S 5$ by putting

$$
\sin ^{2} \chi=\frac{s_{1}-s_{3}}{s-s_{3}}, \quad\left(\frac{r_{1}-r_{2}}{2 a}\right)^{2}=\frac{s_{1}-s_{3}}{\sigma-s_{3}},
$$

and this makes

(4) $\Omega=4 \int_{s_{1}}^{\infty} \frac{\frac{1}{2} V(-\Sigma)}{s-\sigma} \frac{d s}{\sqrt{S}}=4 A\left(f K^{\prime}\right)=2 \pi(1-f)-4 K \mathrm{zn} f K^{\prime}$. 
With the variable $y$ of $\S 29$

$$
1-y=\frac{P N^{2}}{P \bar{Q}^{2}}, \quad \frac{y}{1-y}=\frac{a^{2} \sin ^{2} \theta}{P N^{2}},
$$

and denoting the value of $Y$ for $y=1$ by $Y^{\prime}$,

$$
Y^{\prime}=4\left(y_{1}-1\right)\left(y_{2}-1\right)=-4 \frac{b^{2}}{A^{2}}=-4 \frac{b^{2}}{a^{2}} y_{1} y_{2},
$$

so that from (11) $\S 29$

in which

$$
\begin{aligned}
\Omega & =\int_{0}^{y_{2}} \frac{2 y}{1-y} \frac{b}{a} \frac{\vee\left(y_{1} y_{2}\right) d y}{\vee Y}=\int\left(\frac{1}{1-y}-1\right) \frac{2 \sqrt{ }\left(-Y^{\prime}\right) d y}{\sqrt{ }} \\
& =4 B\left(f^{\prime} K^{\prime}\right)-2 K \sqrt{\frac{-Y^{\prime}}{y_{1}}}
\end{aligned}
$$

(8) $\mathrm{dn}^{2} f^{\prime} K^{\prime}=\frac{\sigma^{\prime}-s_{3}}{s_{1}-s_{3}}=\frac{1}{y_{1}}=\left(\frac{2 A}{r_{1}+r_{2}}\right)^{2}, \quad \mathrm{dn}^{2} f K^{\prime}=\left(\frac{r_{1}-r_{2}}{2 A}\right)^{2}$, from (8) $§ 45$,

$$
\begin{aligned}
& \mathrm{dn}^{2} f^{\prime} K^{\prime} \mathrm{dn}^{2} f K^{\prime}=k^{2}, \quad f+f^{\prime}=1, \\
\Omega= & 2 \pi(1-f)+4 K \mathrm{zn}(1-f) K^{\prime}-4 K \frac{2 b}{r_{1}+r_{2}} \\
= & 2 \pi(1-f)-4 K \mathrm{zn} f K^{\prime},
\end{aligned}
$$

as before in (4).

So also by Lemma II

$$
d V=d x_{\frac{1}{2}} \log \frac{P Q+a \sin \theta}{P Q-a \sin \theta}=a \sin \theta d \theta \operatorname{th}^{-1} \frac{a \sin \theta}{P Q},
$$

and as before in $\S \mathbf{4 8}$ we may transform this into

$$
\text { (12) } \frac{d V}{d \theta}=-\frac{d}{d \theta}\left[(a \cos \theta+A) \operatorname{th}^{-1} \frac{a \sin \theta}{P Q}\right]+\frac{a^{2}+a A \cos \theta}{P Q}-b \frac{d \Omega(P N)}{d \theta} \text {, }
$$

so that, as before in (4) $\S 48$, over the circle,

$$
V+b \Omega=\int_{0}^{2 \pi}(a+A \cos \theta) \frac{a d \theta}{P} \bar{Q}=a \frac{d V}{d a}+A \frac{d V}{d A} .
$$

To connect with the E. F.'s of $h F$ and $2 f F^{\prime}$, by means of $\Omega(M Q)$,

$$
\begin{gathered}
\frac{d \Omega}{d \theta}=\frac{d \Omega(P N)}{d \theta}=\frac{d I_{4}}{d \theta}-d \Omega(M Q), \\
\Omega=2 \pi-\Omega(M Q),
\end{gathered}
$$

as in (4) $\S 49$. 
51. Dissecting by lines $Q Q^{\prime \prime}$ parallel to $A B$ into strips

of which by Lemma IV

$$
Q Q^{\prime \prime} d y=2 a^{2} \cos ^{2} \theta d \theta
$$

(2) $d \Omega=\frac{b d y}{P R^{2}}\left(\frac{Q R}{P \bar{Q}^{ \pm}} \pm \frac{R Q^{\prime \prime}}{P Q^{\prime \prime}}\right)=\left(\frac{a^{2} \cos ^{2} \theta+a A \cos \theta}{P Q}+\frac{a \cos ^{2} \theta-a A \cos \theta}{P Q^{\prime \prime}}\right) \frac{b d \theta}{P R^{2}}$, we may take

$$
\frac{d \Omega}{d \theta}=\frac{a^{2} \cos ^{2} \theta+a A \cos \theta}{P R^{2}} \frac{b}{P Q}=\frac{d \Omega(P R)}{d \theta},
$$

and then integrate round the circle from 0 to $2 \pi$.

So also from Lemma II, with the same dissection,

(4) $d V=d y \log \frac{P Q+P Q^{\prime \prime}+2 a \cos \theta}{P Q+P Q^{\prime \prime}-2 a \cos \theta}=2 d y$ th ${ }^{\circ} \frac{2 a \cos \theta}{P Q+P Q}=2 d y \operatorname{th}^{-1} \frac{P Q-P Q^{\prime \prime}}{2 A}$,

$$
\frac{d V}{d \theta}-2 \frac{d}{d \theta}\left(y \operatorname{th}^{-1} \frac{P Q-P Q^{\prime \prime}}{2 A}\right)=\frac{4 a^{2} A^{2} \sin ^{2} \theta}{4 A^{2}-\left(P Q-P Q^{\prime \prime}\right)^{2}}\left(\begin{array}{c}
1 \\
P Q
\end{array}+\frac{1}{P Q^{\prime \prime}}\right)
$$

$$
\begin{aligned}
& =\frac{1}{2} a^{2} \sin ^{2} \theta \frac{P Q \cdot P Q^{\prime \prime}-A^{2}+a^{2}-b^{2}}{P R}\left(\frac{1}{P Q}+\frac{1}{P Q^{\prime \prime}}\right) \\
& =\left(P R^{2}-b^{2}\right)\left(\frac{a^{2}+a A \cos \theta+b^{2}}{P R^{2} \cdot P Q}+\frac{a^{2}-a A \cos \theta+b^{2}}{P Q^{2} \cdot P R^{\prime}}\right),
\end{aligned}
$$

and here again, in integrating round the circle we can take

$$
V=\int_{0}^{2 \pi}\left(P R^{2}-b^{2}\right) \frac{a^{2}+a A \cos \theta+b^{2}}{P R^{2}} \frac{d \theta}{P Q},
$$

and $V+b \Omega$ is the same as before in $(13) \S 50$.

52. Finally there is the integration for $V$ and $\Omega$ required when the circle is dissected into concentric rings; and now $d V / d r$ is the potential of a circle of radius $r$, and

$$
\begin{gathered}
\frac{d V}{d a}=4 F^{\prime} \frac{a}{r_{1}}=8 K \frac{a}{r_{1}+r_{2}}, \\
V=\int_{0}^{a} 4 F^{\prime} \frac{a}{r_{1}} d a=\int 8 K \frac{a}{r_{1}+r_{2}} d a,
\end{gathered}
$$

of which the result is known already by the other dissections, although the integration appears intractable at first sight.

So also, from $(3) \S 31$

$$
\frac{d \Omega}{d a}=-\frac{d^{2} V}{d a d b}=\frac{4 a b}{r_{1}^{3}} \frac{E}{c^{\prime 2}}=\frac{32 a b}{\left(r_{1}+r_{2}\right)^{3}} \frac{2 H-k^{\prime 2} K}{k^{\prime 4}},
$$

leading to an integral of appearance still more intractable. 
53. Treating the potential $U$ of the solid cylinder in the preceding manner, as given by a function homogeneous in the second degree of $a, A, b$,

$$
2 U=a \frac{d U}{d a}+A \frac{d U}{d A}+b \frac{d U}{d b}
$$

the first term is $a$ times the potential of the curved surface, or skin potential as it may be called of the cylinder, so that

$$
\frac{1}{a} \frac{d U}{d a}=\int_{0}^{2 \pi} \operatorname{th}^{-1} \frac{b}{P Q} d \theta=\int_{t_{3}}^{t_{2}} 2 \mathrm{th}^{-1} \sqrt{\frac{t_{1}-\tau}{t_{1}-t}} \cdot \frac{d t}{V\left(t_{2}-\frac{d t}{\left.t \cdot t-t_{3}\right)}\right.},
$$

not an elliptic integral; but $d U / d A$ and $d U / d b$ are given in $\S \S 44,54$ as the potential of the cylinder due to transverse and longitudinal magnetization.

We shall apply the same method in $\S 64$ to Mr. Coleridge FARR's problem (Proceedings of the Royal Society, November, 1898), to determine the electro-rnagnetic force of a cylindrical coil of finite thickness, where a similar intractable integral is encountered, in conjunction with elliptic integrals, which we consider tractable.

A graphical representation can be given on the Mercator chart of the integral $d W / a d a$ in (2) by denoting the angle $M Q P$ by $\phi$, so that

$$
\operatorname{th}^{-1} \frac{b}{P Q}=\operatorname{th}^{-1} \sin \phi=\log \tan \left(\frac{1}{4} \pi+\frac{1}{2} \phi\right) ;
$$

then if $\phi, \theta$ are taken as latitude and longitude, and the curve drawn on the Mercator chart, the area will represent the integral (2).

Or else $\operatorname{th}^{-1}(b / P Q)$ can be expanded in odd powers of $b / P Q=b /\left(r_{1} \Delta \omega\right)$, and then the integral of each term such as

$$
D_{n}=\int_{0}^{\frac{1}{2} \pi} \frac{d \omega}{(\Delta \omega)^{2 \pi+1}}
$$

can be expressed by means of $F$ and $E$, by means of the recurring relation

$$
(2 n-1) c^{\prime 2} D_{n}-(2 n-2)\left(1+{c^{\prime}}^{2}\right) / D_{n-1}+(2 n-3) D_{n-2}=0,
$$

starting with $D_{0}=F, D_{1}=E / c^{\prime 2}$.

\section{Mutual induction of a circle and coaxial helix.}

54. The III. E. I. arises in the calculation of $M$ between a helix and a coaxial ring; this has been carried out by Viriamu Jones in the Philos ophical Magazine, January, 1889 ; Philosophical Transactions (1891); Proceedings of the Royal Society, December, 1897 ; consult also Webster, Electricity and Magnetism, p. 457 ; MASCART and JOUBERT, Electricity (translated by Atrinson); and Gray, Absolute Measurements in Electricity, volume II, p. 313. 
It results from the form of the integral that $M$ is the same as between the ring and a uniform current sheet in a coaxial cylinder replacing the helix, in which the linear helical current $\gamma_{h}$ and the superficial current $\gamma$ across unit height of the cylinder are connected with $p$ the pitch of the helix by the relation

$$
\gamma_{h}=2 \pi p \gamma \text {. }
$$

For if $N$ denotes the number of windings and $\Theta$ the total angle of the helix of height $b$,

$$
N \gamma_{h}=b \gamma, \quad \text { where } \quad \Theta=\frac{b}{p}, \quad N=\frac{\Theta}{2 \pi}=\frac{b}{2 \pi p} .
$$

The potential of this current sheet has been worked out by Minchin in the Philosophical Magazine, February, 1894, as being the same as the potential of a cylinder magnetized longitudinally, or of two end plates of the cylinder, positive and negative; the expression for the potential of a plate has been cited already in $\S 43$ above.

We notice now that $M$ is the Stokes function of the two end plates, or of the cylinder magnetized longitudinally, and that $M \gamma_{h} / 2 \pi A$ is the magnetic potential in the plane $x 0 z$ at a distance $A$ from the axis of the cylinder magnetized transversely parallel to this plane with intensity $\gamma$; and denoting this magnetic potential by $\Omega^{\prime} \gamma$, and by $W$ the potential of the solid cylinder,

$$
\frac{M \gamma_{h}}{2 \pi A}=\Omega^{\prime} \gamma, \quad \text { or } \quad M p=A \Omega^{\prime} ;
$$

and

(4) $A \Omega^{\prime}=-A \frac{d U}{d x}=\int_{0}^{b} \int_{0}^{2 \pi}-\frac{-a A \cos \theta d \theta d z}{V\left(\alpha^{2}+z^{2}\right)}=\int_{0}^{2 \pi}-a A \cos \theta d \theta \operatorname{th}^{-1} \frac{b}{P Q}$,

the first integral expression given by Viriamu Jones, employing his notation of $\alpha^{2}$ and $\alpha^{2}+z^{2}$ for $M Q^{2}$ and $P Q^{2}$, but using $z$ instead of $x$, and changing his $\theta$ into $\pi-\theta$, to agree with the preceding treatment.

The result for $M p$ is the same whether $2 a$ denotes the diameter of the helix and $2 A$ of the ring, or vice-versa as with Viriamu Jones, being symmetrical in $a$ and $A$.

Integration by parts enables him to replace (4) by

(5) $A \Omega^{\prime}=\int_{0}^{2 \pi} \frac{a^{2} A^{2} b \sin ^{2} \theta d \theta}{a^{2} V\left(\alpha^{2}+b^{2}\right)}=\frac{1}{2} \int\left(-a A \cos \theta+a^{2}\right) \frac{b d \theta}{P Q}-\frac{1}{2}\left(a^{2}-A^{2}\right) \Omega(M Q)$, integrals discussed already in $\S \S 33,49$; and (5) is the equivalent of the double integral

$$
A \Omega^{\prime}=\int_{0}^{b} \int_{0}^{2 \pi} \frac{a^{2} A^{2} \sin ^{2} \theta d \theta d z}{\left(\alpha^{2}+z^{2}\right)^{2}}=\int_{0}^{b} \int_{0}^{\pi} \frac{2 a^{2} d \theta d z}{P Q^{3}},
$$

showing that the magnetic potential $\Omega^{\prime}$ at $P$ of the cylinder magnetized parallel to $O M$ is the same as that of the surface magnetized circumferentially with intensity $a \sin \theta$. 
It is the second form (5) which is reducible immediately to the standard elliptic form, and by the substitution in $\S \S 26,49$, writing

$$
\begin{gathered}
M Q^{2}=\alpha^{2}=A^{2}+2 A a \cos \theta+a^{2}=m^{2}(\tau-t), \\
P Q^{2}=\alpha^{2}+b^{2}=m^{2}\left(t_{1}-t\right),
\end{gathered}
$$

and thence from (5)

$$
\begin{aligned}
& A \Omega^{\prime}=\frac{1}{2} b \int_{t_{3}}^{t_{2}} \frac{m^{4}\left(t_{2}-t\right)\left(t-t_{3}\right)}{m^{2}(\tau-t)} \frac{\frac{d t}{\sqrt{\left(t_{2}-t \cdot t-t_{3}\right)}}}{m \sqrt{ }\left(t_{1}-t\right)} \\
& \quad=m b \int \frac{\left(t_{2}-t\right)\left(t-t_{3}\right) d t}{(\tau-t) V T},
\end{aligned}
$$$$
p M=A \Omega^{\prime}=m b \int\left(t-t_{3}+\tau-t_{2}-\frac{\tau-t_{2} \cdot \tau-t_{3}}{\tau-t}\right) \frac{d t}{\sqrt{T}}
$$

$$
=r_{1} b\left[\int \frac{t-t_{3}}{\sqrt{ }\left(t_{1}-t_{3}\right)} \frac{d t}{\sqrt{ } T}+\frac{\tau-t_{2}}{t_{1}-t_{3}} \int \frac{\sqrt{ }\left(t_{1}-t_{3}\right) d t}{\sqrt{ } T}\right.
$$

$$
\left.-\sqrt{\frac{\tau-t_{2}}{t_{1}-\tau \cdot \tau-t_{3}}} \int \frac{\frac{1}{2} V\left(-T^{\prime}\right)}{\tau-t} \frac{d t}{\sqrt{ } T}\right] \text {. }
$$

Hence, on fig. $A$,

so that

$$
\begin{aligned}
\sqrt{\frac{t_{1}-\tau}{t_{1}-t_{2}}}=\frac{b}{r_{2}} & =\sin \psi, \quad \sqrt{\frac{\tau-t_{2}}{t_{1}-t_{2}}}=\frac{a-A}{r_{2}}=\cos \psi, \\
\sqrt{\frac{\tau-t_{3}}{t_{1}-t_{3}}} & =\frac{a+A}{r_{1}}=\cos \psi^{\prime}=\Delta \psi, \quad \psi=\operatorname{am} 2 f F^{\prime},
\end{aligned}
$$

$$
\begin{gathered}
p M=r_{1} b\left[F-E+F c^{\prime 2} \cos ^{2} \psi-\cot \psi \cos \psi^{\prime} B\left(2 f F^{\prime}\right)\right] . \\
\frac{M}{r_{1} \Theta}=F-E-\cot \psi \cos \psi^{\prime} A(1-2 f) F^{\prime},
\end{gathered}
$$

the equivalent of Viriamu Jones's result in (6) p. 198, Proceedings of the Royal Society, 1898.

We shall replace his angle $\beta$ by $\psi_{1}$ to avoid confusion with Maxwell's modular angle $\beta$, and then

(15) $\psi_{1}=\operatorname{am}(1-2 f) F^{\prime}, \sin \psi_{1}^{\prime}=\frac{\tan \psi^{\prime}}{\tan \psi}=\frac{a-A}{a+A} \frac{r_{1}}{r_{2}}, \cos \psi_{1}^{\prime}=\frac{V(a A)}{\frac{1}{2}(a+A)}$,

so that the angle $\psi_{1}^{\prime}$ is constructed on fig. $A$ by drawing the circle on the diameter $A M$ cutting the axis $O F$ in $G$, and then drawing $O P_{1}$ perpendicular to the radius $G O^{\prime}$ of this circle, then the angle $O A P_{1}$ or $O G O^{\prime}$ is $\psi_{1}^{\prime}$. 
55. With the notation of $\S 47$, equation (5) $\S 54$, is written
(1) $2 A \Omega^{\prime}+\left(a^{2}-A^{2}\right) \Omega(M Q)=\int\left(a^{2}-a A \cos \theta\right) \frac{b d \theta}{P Q}$.
(2) $2 A \Omega^{\prime}+\left(a^{2}-A^{2}\right) \Omega(P Y)=\int\left(a^{2}-a A \cos \theta\right) \frac{b d \theta}{P Q}-\left(a^{2}-A^{2}\right) I_{3}$;

and in a complete revolution of $\theta$ from 0 to $2 \pi$,

$$
\begin{aligned}
\Omega(M Q) & =\int_{0}^{\pi} \frac{M Q^{2}+a^{2}-A^{2}}{M Q^{2}} \frac{b d \theta}{P Q}=b \int \frac{d \theta}{P Q}+\int \frac{a^{2}-A^{2}}{M Q^{2}} \frac{b d \theta}{P Q} \\
& =2 F \frac{b}{r_{1}}+2 B\left(2 f F^{\prime}\right)=2 F c^{\prime} \text { sn } 2 f F^{\prime}+2 B\left(2 f F^{\prime}\right)
\end{aligned}
$$

equivalent to the result in $(10) \S 49$.

Also $I_{3}$ vanishes in a complete revolution of $\theta$, and from (16) $\S 47$,

so that

$$
\Omega(P Y)=\Phi=4 B\left(f K^{\prime}\right),
$$

in which

$$
\begin{aligned}
2 A \Omega^{\prime}+4\left(a^{2}-A^{2}\right) B\left(f K^{\prime}\right) & =\int_{0}^{2 \pi}\left(a^{2}-a A \cos \theta\right) \frac{b d \theta}{P Q} \\
& =\frac{8 a^{2} b K}{r_{1}+r_{2}}+2 b\left(r_{1}+r_{2}\right)(K-H),
\end{aligned}
$$

(6) $p M=A \Omega^{\prime}=b\left(r_{1}+r_{2}\right) K \mathrm{dn}^{2} f K^{\prime}+b\left(r_{1}+r_{2}\right)(K-H)$

$$
-2\left(a^{2}-A^{2}\right) B\left(f K^{\prime}\right),
$$

in the form given in $(5) \S 44$, the quadric transformation of (13) $\S 54$.

This again, by means of $(2),(3), \S 17$, can be transformed into

$$
\frac{M}{\left(r_{1}+r_{2}\right) \Theta}=K-H+\frac{\mathrm{cn} 2 f K^{\prime}}{\operatorname{sn} 2 f K^{\prime}} C(1-2 f) K^{\prime} .
$$

In the indeterminate case of $2 f=1$ and $C(1-2 f) K^{\prime}=\infty$, we have $A=a$; but returning to the integral in (10) $\$ 54$, and putting $\tau=t_{2}$,

(8) $p M=A \Omega^{\prime}=\frac{m b}{a} \int \frac{\left(t-t_{3}\right) d t}{\sqrt{ } T}=b m \sqrt{ }\left(t_{1}-t_{3}\right)(F-E)=r_{1} b(F-E)$,

$$
\frac{M}{\Theta}=r_{1}(F-E)=\left(r_{1}+r_{2}\right)(K-H+k K),
$$

and this is the coefficient of self induction of the helix on a final turn of itself. 
Numerical calculation of the mutual induction.

56. The numerical calculation of $M$ for given dimensions $a, A, b$ now requires the five operations following, in the numerical tables of F. E., vol. II, LEGENDRE, and it is useful to check the calculation on fig. $A$, drawn to scale.

I. Calculate $\psi$ and $\psi^{\prime}$ from

and then

$$
\tan \psi=\frac{b}{a-A}, \quad \tan \psi^{\prime}=\frac{b}{a+A} ;
$$

$$
r_{1}=(a+A) \sec \psi^{\prime}, \quad r_{2}=(a-A) \sec \psi .
$$

II. Calculate $\psi_{1}$ and the modular angle $\gamma$ from

$$
\sin \psi_{1}=\frac{\cos \psi}{\cos \psi^{\prime}}, \quad \cos \gamma=\frac{\sin \psi^{\prime}}{\sin \psi} .
$$

III. Calculate $f$ by Legendre's Table IX from

$$
2 f=\frac{F\left(\psi, c^{\prime}\right)}{F\left(\frac{1}{2} \pi, c^{\prime}\right)}, \quad 1-2 f=\frac{F\left(\psi_{1}, c^{\prime}\right)}{F\left(\frac{1}{2} \pi, c^{\prime}\right)} .
$$

IV. Calculate zn $(1-2 f) F^{\prime}$ by Table IX from

$$
\operatorname{zn}(1-2 f) F^{\prime}=E\left(\beta^{\prime}, c^{\prime}\right)-(1-2 f) E\left(\frac{1}{2} \pi, c^{\prime}\right) \text {. }
$$

V. Look out

$$
F=F\left(\frac{1}{2} \pi, c\right), \quad E=E\left(\frac{1}{2} \pi, c\right) .
$$

When $f$ is one of the rational fractions of (1) $\S 4$, we can express $c, c^{\prime}$, sn $2 f F^{\prime}$, zn $2 f F^{\prime}, \ldots$, as algebraical functions of a parameter, leaving $F$ and $E$ as the only transcendents, and these are tabulated by LEGENDRE in his Table I to an extra degree of accuracy.

In the numerical application of Viriamu Jones, Philosophical Transactions (1891), Proceedings of the Royal Society (1897),

$$
\begin{gathered}
2 a=21.02673, \quad 2 A=13.01997, \quad 2 b=5.02480 \text { (inches), } \\
a-A=4.00338, \quad a+A=17.02335, \quad b=2.51240 ;
\end{gathered}
$$

and working with four figure logarithms we find $\psi=32^{\circ} 3^{\prime}, \psi^{\prime}=8^{\circ} 24^{\prime}$, $\gamma=74^{\circ} 1^{\prime}, \psi_{1}=58^{\circ} 58^{\prime}$; or to the nearest degree, $\gamma=74^{\circ}, \psi_{1}=59^{\circ}$, thus avoiding proportional parts in Legendre's Table IX; $F=2.70807$, $E=1.08443$, and $F^{\prime \prime}=1.60198, E\left(\psi_{1}, c^{\prime}\right)=1.04123,1-2 f=0.6500$, $f=0.1750, \quad \pi f=0.54985, \quad E^{\prime}=1.54052, \quad(1-2 f) E^{\prime}=1.00134$, $E \psi_{1}=1.01847, \quad \operatorname{zn}(1-2 f) F^{\prime}=0.01713, \quad F^{\prime} \mathrm{zn}(1-2 f) F^{\prime}=0.04638$, $A(1-2 f) F^{\prime}=0.50347, \cot \psi \cos \psi^{\prime} A(1-2 f) F^{\prime}=0.7952, F-E=1.6236$, $M / r_{1} \Theta=0.8284, \Theta=201 \pi, r_{1}=17.21, M=9001$ inches, as against 9028 inches, given by Viriamu Jones. 
This rough calculation is retained for the purpose of showing the term requiring extra calculated figures, compared with the parts where fewer figures are retained for the same degree of accuracy; thus of the three chief terms of $M / r_{1} \Theta$, the first $F-E=1.6236$ is about double the second $\pi f \cot \psi \cos \psi^{\prime}=0.8638$, while the remainder $\cot \psi \cos \psi^{\prime} F$ zn $(1-2 f) F^{\prime}=0.0734$.

The modular angle must thus be determined with extra accuracy for the determination of $F-E$; and in this case raising the modular angle by one minute to $74^{\circ} 1^{\prime}$ will make $F-E=1.6258$, and raise $M$ to 9024 .

A diminution of 0.1 per cent. in $f$ in the second term will raise $M$ about 0.05 per cent. to about 9028.5 , without affecting the last term appreciably.

Variation of the induction due to change in the dimensions.

57. Viriamu JoNes calculates the effect of small error of measurement $d A, d a, d b$ in $A, a, b$ in giving a change $d M$ in $M$, such that

$$
d M=\left(A \frac{\partial M}{\partial A}\right) \frac{d A}{A}+\left(a \frac{\partial M}{\partial a}\right) \frac{d a}{a}+\left(b \frac{\partial M}{\partial b}\right) \frac{d b}{b},
$$

so that 1 per cent. change in $A, a, b$ gives a change in $M$ of

$$
\frac{A}{100} \frac{\partial M}{\partial A}, \quad \frac{a}{100} \frac{\partial M}{\partial a}, \quad \frac{b}{100} \frac{\partial M}{\partial b} ;
$$

and their sum is $M / 100$, because $M$ is a homogeneous function of the variables $A, a, b$, and of the first degree, the total helical angle $\Theta$ or number of windings $\Theta \div 2 \pi$ being kept constant. But if the pitch $p$ of the helix is kept constant, $M$ is of the second degree in $A, a, b$.

Differentiating the double integral expression for $M$ in (4) $\S 54$,

$$
M=\frac{\Theta}{b} \int_{0}^{b} \int_{0}^{2 \pi}-\frac{A a \cos \theta d \theta d z}{V\left(\alpha^{2}+z^{2}\right)},
$$

and supposing the limits of $\theta$ to be 0 and $2 \pi$ in the sequel, we have

$$
\begin{aligned}
& \frac{\partial M}{\partial A}=\frac{\Theta}{b} \iint \frac{-a \cos \theta d \theta d z}{V\left(\alpha^{2}+z\right)}+\frac{\Theta}{b} \iint \frac{(A+a \cos \theta) A a \cos \theta d \theta d z}{\left(\alpha^{2}+z^{2}\right)^{\frac{2}{2}}} \\
& A \frac{\partial M}{\partial A}=M+\Theta \int \frac{\left(A^{2}+A a \cos \theta\right) A a \cos \theta d \theta}{\alpha^{2} V\left(\alpha^{2}+b^{2}\right)}=\Theta A^{2} \int \frac{a^{2}+a A \cos \theta}{M Q^{2}} P \bar{Q}
\end{aligned}
$$

and similarly

$$
a \frac{\partial M}{\partial a}=M+\Theta \int \frac{\left(A a \cos \theta+a^{2}\right) A a \cos \theta d \theta}{\alpha^{2} \sqrt{ }\left(\alpha^{2}+b^{2}\right)}=\Theta a^{2} \int \frac{a A \cos \theta+A^{2}}{M Q^{2}} \frac{d \theta}{P Q}
$$


so that

$$
a \frac{d M}{d a}+A \frac{d M}{d A}=2 M-\Theta \int \frac{-a A \cos \theta d \theta}{P Q},
$$

while, keeping $\Theta$ constant,

and then

$$
\begin{aligned}
& b M=\Theta \int_{0}^{b} \int \frac{-a A \cos \theta d \theta d z}{\sqrt{ }\left(\alpha^{2}+z^{2}\right)}, \\
& b \frac{d M}{d b}+M=\Theta \int \frac{-a A \cos \theta d \theta}{P Q},
\end{aligned}
$$

$$
a \frac{d M}{d a}+A \frac{d M}{d A}+b \frac{d M}{d b}=M,
$$

a verification of the homogeneity. Also

$$
\begin{gathered}
\frac{1}{A} \frac{d M}{d A}+\frac{1}{a} \frac{d M}{d u}=\Theta \int \frac{d \theta}{P Q}=4 F \frac{\Theta}{r_{1}}=4 F^{\prime} \frac{\sin \psi^{\prime}}{p}, \\
\frac{1}{A} \frac{d M}{d A}-\frac{1}{a} \frac{d M}{d a}=\Theta \int \frac{a^{2}-A^{2}}{M Q^{2}} \frac{d \theta}{P Q}=\frac{4 B\left(2 f F^{\prime}\right)}{p}, \\
\frac{1}{A} \frac{d M}{d A}=\frac{2}{p}\left[F c^{\prime} \operatorname{sn} 2 f F^{\prime}+B\left(2 f F^{\prime}\right)\right]=\frac{4 B\left(f K^{\prime}\right)}{p} \\
\frac{1}{a} \frac{d M}{d u}=\frac{2}{p}\left[F c^{\prime} \operatorname{sn} 2 f F^{\prime}-B\left(2 f F^{\prime}\right)\right]=-\frac{4 A(1-f) K^{\prime}}{p},
\end{gathered}
$$

and we see now that the conditions are satisfied that this $M$ of Viriamu Jones is the Stokes function of Minchis's pair of end circular plates of density $\gamma= \pm 1 / 2 \pi p$, corresponding to a helical current $\gamma_{h}=1$, and

(16) $p M=-2 a^{2} \cdot A(1-f) K^{\prime}+2 A^{2} \cdot B\left(f K^{\prime}\right)+b\left(r_{1}+r_{2}\right)(K-H)$, in agreement with $(6) \S 55$ above.

58. Thus the magnetic components for the helical current $\gamma_{h}$, axial and transversal, are, for magnetic potential $\Omega$,

$$
\frac{d \Omega}{d b}=-\frac{\dot{\gamma_{h}}}{2 \pi A} \frac{d M}{d A}=-4 \gamma B\left(f K^{\prime}\right)=-\gamma\left(2 \pi f+4 K \mathrm{zn} f K^{\prime}\right),
$$

and, now keeping $p$ constant in (8) $\S 57$,

$$
\begin{aligned}
\frac{d \Omega}{d A} & =\frac{\gamma_{h}}{2 \pi A} \frac{d M}{d b}=\frac{\gamma}{A} \int_{0}^{2 \pi} \frac{-a A \cos \theta d \theta}{P} \bar{Q} \\
& =4 \gamma \frac{r_{1}+r_{2}}{2 A}(K-H)=4 \gamma \operatorname{dn} f K^{\prime} \frac{K-H}{k}=4 \gamma \frac{K-H}{\operatorname{dn}(1-f) K^{\prime}} .
\end{aligned}
$$


With $b=\infty$ we must take $t_{1}=\infty$, and the integrals in (10) $\S 54$ reduce to

$$
\begin{aligned}
& p M=A \Omega^{\prime}=m^{2} \int_{t_{3}}^{t_{2}} \frac{t_{2}-t \cdot t-t_{3}}{\tau-t} \frac{d t}{\sqrt{\left(4 \cdot t_{2}-t \cdot t-t_{3}\right)}} \\
& =m^{2} \int\left(t-t_{3}+\tau-t_{2} \frac{\tau-t_{2} \cdot \tau-t_{3}}{\tau-t}\right) \frac{d t}{\sqrt{ }\left(4 \cdot t_{2}-t \cdot t-t_{3}\right)}
\end{aligned}
$$

with

$$
=m^{2}\left[\frac{t_{2}-t_{3}}{2}+\tau-t_{2}-V\left(\tau-t_{2} \cdot \tau-t_{3}\right)\right] \frac{1}{2} \pi
$$

(5) $4 a A=m^{2}\left(t_{2}-t_{3}\right), \quad(a \sim A)^{2}=m^{2}\left(\tau-t_{2}\right), \quad(a+A)^{2}=m^{2}\left(\tau-t_{3}\right)$, so that

$$
p M=\pi A^{2}(A<a) \quad \text { or } \quad \pi a^{2}(A>a) .
$$

Otherwise, with $b=\infty$ in (5) $\S 54$,

$$
p M=A \Omega^{\prime}=\int_{0}^{2 \pi} \frac{a^{2} A^{2} \sin ^{2} \theta d \theta}{a^{2}+2 a A \cos \theta+A^{2}}=\pi A^{2} \text { or } \pi a^{2},
$$

employing the substitution of $\S 26$.

Thus for the infinite helix, in the plane of the end $A B$,

and at $M$ or $M^{\prime}$,

$$
\frac{d \Omega}{d b}=-\frac{\gamma_{h}}{p}=-2 \pi \gamma \quad \text { or } \quad O
$$

$$
\begin{array}{ccc}
\frac{d \Omega}{d A}=4 \gamma \frac{K_{1}-H_{1}}{k_{1}} & \text { or } & 4 \gamma\left(K_{1}-H_{1}\right), \\
k_{1}=\frac{O M}{O A} & \text { or } & \frac{O A}{O M^{\prime}} .
\end{array}
$$

Thence by subtraction, for a helix extending from $b$ to infinity,

$$
\begin{gathered}
\frac{d \Omega}{d b}=4 \gamma A\left(f K^{\prime}\right), \\
\frac{d \Omega}{d A}=4 \gamma\left[\frac{K-H}{d \ln (1-f) K^{\prime}}-\frac{K_{1}-H_{1}}{k_{1}}\right],
\end{gathered}
$$

with $2 f \lessgtr 1$, according as $A \lessgtr a$. 


\section{Potential energy of two coaxial helices.}

59. There remains still the evaluation of the potential energy of two coaxial helical currents or of their equivalent cylindrical current sheets, given by $\mathrm{V}$. Jones in $\S 13$, p. 202, Proceedings of the Royal Society, 1897, and this is the same as the mutual potential energy of the two pairs of equivalent end plates. Viriamu Jones shows that this energy depends on the integral

$$
F(z)=\frac{A a}{p} \int_{0}^{2 \pi} \cos \theta d \theta f(z),
$$

$f(z)=z \log \left[\sqrt{ }\left(1+\frac{z^{2}}{\alpha^{2}}\right)+\frac{z}{\alpha}\right]-\sqrt{ }\left(\alpha^{2}+z^{2}\right), f^{\prime}(z)=\left[\log \sqrt{ }\left(1+\frac{z^{2}}{\alpha^{2}}\right)+\frac{z}{\alpha}\right]$

$$
F(z)=M z+\int_{0}^{2 \pi} \frac{-A a \cos \theta}{p} d \theta \vee\left(\alpha^{2}+z^{2}\right),
$$

and the second term in $F(z)$,

$$
\begin{aligned}
H(z) & =\frac{m^{3}}{p} \int_{t_{3}}^{t_{2}}\left(2 t-t_{2}-t_{3}\right)\left(t_{1}-t\right) \frac{d t}{\sqrt{T}} \\
& =\frac{m^{3}}{p} \int\left(\left(t_{2}-t \cdot t-t_{3}\right)-\vee T \frac{d}{d t} \vee T\right) \frac{d t}{\sqrt{ } T},
\end{aligned}
$$

the same integral as for $G$ in $\S 32$.

But it is the force between the two helices which alone is required, and this is given by $d F(z) / d z$, or by the change in $F(z)$ due to a small relative axial displacement of the helices, and as this is equivalent to the removal of a circular element from one end to the other of a helix, this force depends only on the difference of the values of $M$ between one helix and the two circular ends of the other helix ; and this is calculated by the preceding analysis, which gives the value of $M$ as a function, say $M(b)$, of $b$ the height of one helix say of radius $A$, when the ring of radius $a$ is in the plane of one base of the helix.

When the circle is at a distance $z$ from the mean section of a helix of height $h$

$$
M=M\left(z+\frac{1}{2} h\right)-M\left(z-\frac{1}{2} h\right) ;
$$

and since $M(-z)=-M(z)$, this makes $M=2 M\left(\frac{1}{2} h\right)$, in the mean section.

The hydrodynamical analogy is complete, $M$ being the Stokes function, and $V\left(z+\frac{1}{2} h\right)-V\left(z-\frac{1}{2} h\right)$ the velocity function, $V(z)$ denoting the potential of a circular plate, due to a uniform distribution of source or sink over the plate, thereby producing a streaming motion circulating through the helix, a line of flow having $M=$ constant: thereby we gain a physical conception of the lines of magnetic force inside and outside a solenoid.

MAXweLL's figure XVIII can be utilised by the method of superposition for drawing these lines when the ring is made to expand into a cylinder by progressive axial displacement. 


\section{Exploration of the electromagnetic field.}

60. The exploration of the electromagnetic field of the ring current is given by MAXweLl, E. and M. $\S 702$, and in fig. XVIII by drawing the twelve surfaces $\Omega=2 \pi f$, taking

$$
f=(0,1,2,3, \ldots, 11,12) \frac{1}{12} ;
$$

equivalent to direction at the ring on a clock face at $B$, for every hour and half hour from XII to VI, and again from VI to XII.

Any surface $\Omega=2 \pi f$ will cross the axis $O z$ at a point $H$ such that

$$
\sin \psi=\sin O B H=\frac{O H}{H B}=1-\frac{2 \pi f}{2 \pi}=1-f,
$$

so that $H$ is determined geometrically by drawing a circle center $A$ and radius $(1-f) A B$, cutting the circle on diameter $A B$ in $H^{\prime}$, and joining $B H^{\prime}$ cutting $O z$ in $H$.

Thence at $O$, where $f=1$,

$$
\frac{d \Omega}{d b}=\frac{2 \pi(1-f)}{a(1-f)}=\frac{2 \pi}{a},
$$

the galvanometer constant for the ring.

Practically we need only draw the surfaces from $f=0$ to $\frac{1}{2}$, or between XII and III o'clock, as the remainder from III to VI can be inferred by the theorems in (25) that $O M \cdot O M^{\prime}=O B^{2}$ for points $P, P^{\prime}$ on a line through $B$.

In another method of exploration we can utilize the analytical results given in Philosophical Transactions, 1904, and determine the value of $\Omega, V$, and $M$ in $\S \S 34,42,43,44,54$, when $2 f$ is one of the simplest rational fractions, such as

$$
2 f=1, \frac{2}{3}, \frac{1}{2}, \frac{1}{3}, \frac{1,3}{4}, \frac{1}{5}, \frac{1}{6}, \ldots
$$

In this method it is simple to work with the elliptic functions to modulus $c^{\prime}$ and argument $2 f F^{\prime}$ and to use the quadric transformation to obtain the result to argument $f K^{\prime}$, if required.

A curve along which $2 f$ is a rational fraction is an algebraical curve, passing through $A$ at an angle $f \pi$ with $O x$, and having $O z$ as an asymptote.

We proceed to trace a few of the simplest, and for this purpose it is useful to employ a stereographic chart with poles at $A$ and $B$, and center of projection the antipodes of $O$, in which case, as in $\S 25$,

$$
k=\tan \frac{1}{2} \lambda=\operatorname{th} \alpha, \quad c^{\prime}=\tan \left(45^{\circ}-\frac{1}{2} \lambda\right)=e^{-2 \alpha},
$$

$\lambda$ denoting the latitude, the angle $F O L$ on fig. $A$. 
Exploring the values of $\Omega, V, M$ at the principal divisions of the clock face: XII o'clock, $\quad f=0, \quad b=0, \quad \psi=0, \quad x=a k=a \frac{1-c^{\prime}}{1+c^{\prime}}$,

$$
\Omega=2 \pi, \quad V=16 a \frac{E+c^{\prime} F}{1+c^{\prime}}, \quad M=0 .
$$

VI o'clock, $\quad f=1, \quad b=0, \quad \psi=\pi, \quad x=\frac{a}{k}=a \frac{1+c^{\prime}}{1-c^{\prime}}$,

$$
\Omega=0, \quad V=16 a \frac{E-c^{\prime} F}{1-c^{\prime}}, \quad M=0 .
$$

III o'clock, $2 f=1, \quad \psi=\frac{1}{2} \pi, \quad x=a, \quad z=2 a \frac{c^{\prime}}{c}, \quad \sin \psi^{\prime}=c^{\prime}, \quad \psi=2 \operatorname{am} \frac{1}{2} K^{\prime}-\frac{1}{2} \pi$, $\Omega=\pi-2 F c^{\prime}, \quad V=4 a \frac{H}{c}-\pi z=z\left(\frac{2 H}{c^{\prime}}-\pi\right), \quad \frac{M}{\Theta}=2 a \frac{F-E}{c}=z \frac{F-E}{c^{\prime}}$, on reference to $(9) \S 55$.

II o'clock, $2 f=\frac{2}{3}$; and (see Philosophical Transactions, 1904, page 261 ) in region $B, 3>p>1$,

$$
\begin{array}{cc}
c^{2}=\frac{(p-1)^{3}(p+3)}{16 p}, & c^{\prime 2}=\frac{(p+1)^{3}(-p+3)}{16 p}, \\
\text { zn } \frac{2}{3} F^{\prime}=\frac{3-p}{6} \vee p, & \text { zn } \frac{2}{3} F^{\prime \prime}=\frac{3-p}{6} \vee p,
\end{array}
$$

$\cos \psi=\operatorname{cn} \frac{2}{3} F^{\prime}=\frac{p-1}{p+1}, \quad \cos \psi^{\prime}=\mathrm{dn} \frac{2}{3} F^{\prime \prime}=\frac{p-1}{2}, \quad \sec \psi-\sec \psi^{\prime}=1$, along the curve, analogous to

$$
\psi+\psi^{\prime} \text {, or } \tan \psi+\tan \psi^{\prime} \text {, or } \cos \psi+\cos \psi^{\prime}, \ldots \text { a constant, }
$$

a circle, or parabola, or magnetic line of force, $\cdots$ in biangular coördinates $\psi, \psi^{\prime}$. I o'clock :

$$
2 f=\frac{1}{3}, \quad \sin \Psi=\operatorname{sn} \frac{1}{3} F^{\prime}=\frac{2}{p+1},
$$

$$
\sin \Psi^{\prime}=c^{\prime} \operatorname{sn} \frac{1}{3} F^{\prime \prime}=\sqrt{\frac{p+1 \cdot-p+3}{4 p}}, \quad \text { zn } \frac{1}{3} F^{\prime \prime}=\frac{9-p^{2}}{12 \sqrt{ } p} .
$$

I. $30,2 f=\frac{1}{2}, \quad \psi=\operatorname{am} \frac{1}{2} F^{\prime \prime}, \quad \sin \psi=\sqrt{\frac{1}{1+c}}, \quad \sin \psi^{\prime}=\sqrt{ }(1-c)$,

$$
\tan ^{2} \psi-\tan ^{2} \psi^{\prime}=1, \quad \frac{z^{2}}{(a-x)^{2}}-\frac{z^{2}}{(a+x)^{2}}=1,
$$

zn $\frac{1}{2} F^{\prime}=\frac{1}{2}(1-c), \quad \Omega=\frac{3}{2} \pi-F(1-c)-2 F \sqrt{ }(1-c)$. 
XII. 45, or II. $15,2 f=\frac{1}{4}$ or $\frac{3}{4}$ (Philosophical Transactions, page 478),

$$
\begin{gathered}
1>p>\sqrt{ } 2-1, \quad \vee c=\frac{1}{2}\left(\frac{1}{p}-p\right), \\
\cos ^{2} \psi^{\prime}=\operatorname{dn}^{2} \frac{1}{4} F^{\prime \prime}=\frac{(1+p)^{3}(1-p)}{4 p}, \quad \sin ^{2} \psi^{\prime}=\frac{\left(1+p^{2}\right)\left(-1+2 p+p^{2}\right)}{4 p}, \\
\tan ^{2} \psi=\frac{4 p^{3}}{(1+p)(1-p)^{3}}, \quad \operatorname{zn} \frac{1}{4} F^{\prime \prime}=\frac{\left(1+2 p+3 p^{2}\right)\left(-1+2 p+p^{2}\right)}{16 p^{2}},
\end{gathered}
$$

and a change of $p$ into $-1 / p$ will change $2 f$ from $\frac{1}{4}$ to $\frac{3}{4}$.

XII. 36, I. 12, I. 48, II. $24 ; 2 f=\frac{1}{5}, \frac{2}{5}, \frac{3}{5}, \frac{4}{5}$, and the results in Philosophical Transactions, page 264 , to be consulted.

XII. 30, II. 30, $2 f=\frac{1}{6}, \frac{5}{6}$; consult page 283 .

61. In an apparatus constructed by Professor Ayrton and Viriamu Jones;

$$
2 A=8, \quad 2 a=13 \text { inches; }
$$

and then for the height $b$ we shall find in correspondence with $2 f$,

For if

$$
\begin{aligned}
& 2 f \mid 0, \quad \frac{1}{4}, \quad \frac{1}{3}, \quad \frac{1}{2}, \quad \frac{2}{3}, \quad \frac{3}{4}, \quad 1 \\
& b \mid 0,1.36,1.47,2.57,4.59,9.79, \infty \text {. }
\end{aligned}
$$

$$
2 f=\frac{1}{2}, \quad b=\frac{a^{2}-A^{2}}{2 \sqrt{ }(a A)}=2.574,
$$

(2) $2 f=\frac{2}{3}$,

$$
\left(\frac{a-A}{a+A}\right)^{2}={c^{\prime}}^{2} \operatorname{sn}^{2}(1-2 f) F^{\prime}=\frac{(p+1)(-p+3)}{4 p},
$$

$$
p=2.8322, \quad \frac{b}{a-A}=\operatorname{tn} \frac{2}{3} F^{\prime}=\frac{2 \vee p}{p-1}, \quad b=4.593,
$$

(3)

$$
\begin{gathered}
2 f=\frac{1}{3}, \quad \frac{2 V a A}{a+A}=\operatorname{dn} \frac{2}{3} F^{\prime}=\frac{p-1}{2}, \quad p=2.9428, \\
\frac{b}{a-A}=\operatorname{tn} \frac{1}{3} F^{\prime}=\frac{2}{V^{\prime}(p-1 \cdot p+3)}, \quad b=1.471, \\
2 f=\frac{3}{4}, \quad \operatorname{dn}^{2} \frac{1}{4} F^{\prime}=\frac{4 a A}{(a+A)^{2}}=\frac{416}{441}=\frac{(1+p)^{3}(1-p)}{4 p},
\end{gathered}
$$

a quartic for $p$ having a root $p=0.43$ and making

$$
\begin{gathered}
c=\frac{1}{4}\left(\frac{1}{p}-p\right)^{2}=0.719, \quad c^{\prime}=0.695, \\
\cos ^{2} \psi^{\prime}=\operatorname{dn}^{2} \frac{3}{4} F^{\prime}=\frac{4}{4} \frac{1}{16} c^{2}=0.547 \\
b=(a+A) \tan \Psi^{\prime}=9.79 .
\end{gathered}
$$


Similarly for $2 f=\frac{1}{4}, b=1.364$.

62. To determine the proportions of the apparatus when the modular angle $\gamma$ is raised to $75^{\circ}$, and we take $2 f=\frac{1}{3}$, as in fig. $A$, we put

$$
c^{2}=\frac{(p-1)^{3}(p+3)}{16 p}=\sin ^{2} 75^{\circ}=\frac{2+\sqrt{ } 3}{4},
$$

of which one root is $p=-\sqrt{ } 3$, but this root leads to

$$
\text { sn } \frac{1}{3} F=\sqrt{ } 3-1, \quad \text { dn } \frac{1}{3} F=\frac{1}{2} \sqrt{ } 2 .
$$

But with the other real root,

(8) $r_{1}=(a+A) \sec \psi^{\prime}=(a+A) \operatorname{dn} \frac{1}{3} F^{\prime}=(a+A) \sqrt{\frac{2 p}{p-1 \cdot p+3}}$,

$$
\begin{aligned}
& p=\frac{\left(z^{2}+1\right)^{2}}{V^{3}}=\left(\frac{\nabla^{2}+1}{v^{2}-1}\right)^{\frac{1}{2}}=2.948661, \\
& \csc \psi=\operatorname{sn} \frac{1}{3} F^{\prime \prime}=\frac{1}{2}(p+1)=1.97433 \quad\left(\psi=30^{\circ} 26^{\prime}\right),
\end{aligned}
$$

$$
\sin \psi^{\prime}=c^{\prime} \sin \psi=\sqrt{\frac{p+1 .-p+3}{4 p}}=0.1319 \quad\left(\psi^{\prime}=7^{\circ} 34^{\prime}\right),
$$

$$
\sin \psi_{1}=\operatorname{sn} \frac{2}{3} F^{\prime}=\frac{2 \sqrt{ } p}{p+1} \quad\left(\psi_{1}=60^{\circ} 26^{\prime}\right),
$$

$$
\cos \psi_{1}^{\prime}=\operatorname{dn} \frac{2}{3} F^{\prime}=\frac{1}{2}(p-1)=0.97433 \quad\left(\psi_{1}^{\prime}=13^{\circ} 13^{\prime}\right),
$$

(11) $F=2.768063145, \quad E=1.076405113, \quad F-E=1.691658032$.

These angles are contrasted with those of the apparatus of Viriamu Jones in the table following.

\begin{tabular}{c|c|c}
$2 f$ & $\frac{1}{3}=0.33$ & 0.35 \\
\hline $\sin ^{-1} c=\gamma$ & $75^{\circ}$ & $74^{\circ} 1^{\prime}$ \\
$\sin ^{-1} k=\beta$ & $36^{\circ} 4^{\prime}$ & $34^{\circ} 36^{\prime}$ \\
$\psi$ & $30^{\circ} 26^{\prime}$ & $32^{\circ} 3^{\prime}$ \\
$\psi^{\prime}$ & $7^{\circ} 34^{\prime}$ & $8^{\circ} 24^{\prime}$ \\
$\psi_{1}$ & $60^{\circ} 26^{\prime}$ & $58^{\circ} 58^{\prime}$ \\
$\psi_{1}^{\prime}$ & $13^{\circ} 3^{\prime}$ & $13^{\circ} 36^{\prime}$ \\
$A / a$ & 0.63196 & 0.61921
\end{tabular}


63. If the windings of the helix are not complete, or if the current is considered through a fraction of the circular ring, the theta functions are required or the pseudo-elliptic algebraical III. E. I. can be employed for a rational value of the fraction $f$, the simplest cases of which will be found worked out in the Philosophical Transactions, 1904.

Lord RAYLEIGH has shown, in a Report to the British Association (1899), that all error in extreme cases of this assumption as between two coaxial helices is eliminated by taking an average for different symmetrical positions.

One object of the present memoir is to see if by a slight change in one of the dimensions of a helix, say the height $b$, designed for the experimental determination of the ampere, it would be possible to make $2 f$ one of the rational fractions given above, and so simplify and check the numerical work.

Or we may construct the apparatus so as to make the angle $\gamma$ in fig. $A$ or $\beta$ in fig. $B$ an integral number of degrees, so that the value of $F$ and $E$ or $K$ and $H$ can be taken out of LEgendRE's Table I and IX without use of proportional parts, and utilise all the decimal figures.

\section{Potential and Stokes function of a solid coil.}

64. To solve Coleridge FARR's problem for a solid cylindrical coil, we have to determine the potential $W$ and Stokes function $P$ of a series of coaxial helical currents or equivalent cylindrical current sheets filling up a solid cylinder, integrating with respect to the radius the expression for a cylindrical sheet.

It is simpler to determine $W$ for a coil stretching in fig. $A$ from the plane $A B$ to infinity on the right, as this is equivalent to integrating over a single circular plate $A B$ of superficial density $\gamma=\gamma_{h} / 2 \pi p$ (qualified as shown in (8) by the factor $a-r, r$ referring to any internal radius $O Q^{\prime}$ ), the influence of the other end plate at infinity being insensible.

In $\S 48$ we have found for the potential $V$ of the plate $A B$ of unit density

$$
\frac{d V}{d \theta}=P Q-P O-A \cos \theta \cdot 2 \operatorname{th}^{-1} \frac{a}{P Q+P O},
$$

the equivalent of $d^{2} W / d a d \theta$, and integrating by parts with respect to $a$,

$$
\begin{aligned}
\frac{d W}{d \theta}= & \int_{0}^{a}(P Q-P O) d a-A \cos \theta \int 2 \operatorname{th}^{-1} \frac{a}{P Q+P O} d a \\
= & \left(\frac{1}{2} a+\frac{3}{2} A \cos \theta\right) P Q-\left(a+\frac{3}{2} A \cos \theta\right) P O \\
& \quad-2 \mathrm{th}^{-1} P \frac{a}{P Q+P O}\left(\frac{1}{4} A^{2}-\frac{1}{2} b^{2}+a A \cos \theta+\frac{3}{4} A^{2} \cos 2 \theta\right) .
\end{aligned}
$$


Integrating again with respect to $\theta$ from 0 to $2 \pi$, the term

$$
I=\int 2 \operatorname{th}^{-1} \frac{a}{P Q+P O} d \theta
$$

is not an elliptic integral, and so considered intractable, like the integral (2) in $\S 53$, to be denoted by $I_{1}$; but

$$
\begin{array}{r}
\int a A \cos \theta \cdot 2 \mathrm{th}^{-1} \frac{a}{P Q+P O} d \theta=a \int\left(1-\frac{b^{2}}{P Z^{2}}\right)\left(a A \cos \theta+A^{2}+b^{2}\right) \frac{d \theta}{P Q} \\
-a V\left(A^{2}+b^{2}\right) \int\left(1-\frac{b^{2}}{P Z^{2}}\right) d \theta
\end{array}
$$

$$
\begin{aligned}
& =\int\left(a A \cos \theta+A^{2}+b^{2}\right) a \frac{d \theta}{P Q}+a b\left[(2 \pi-\Omega(P Z)]-2 \pi a V\left(A^{2}+b^{2}\right) .\right. \\
& \int A^{2} \cos 2 \theta \cdot 2 \operatorname{th}^{-1} \frac{a}{P Q+P O} d \theta=-a A^{2} \int \frac{\sin ^{2} \theta d \theta}{P Q}
\end{aligned}
$$

$$
+\left(A^{2}+b^{2}\right) \int(a+A \cos \theta) \frac{d \theta}{P Q}-b_{V}\left(A^{2}+b^{2}\right) \Omega_{1}(P Z),
$$

so that $W$ is composed of

$$
\left(\frac{1}{2} b^{2}-\frac{1}{4} A^{2}\right) I, \quad-a b[2 \pi-\Omega(P Z)], \quad \frac{3}{4} b V\left(A^{2}+b\right) \Omega_{1}(P Z),
$$

and I. and II. E. I.'s, amounting to employing the theorems of $\S 33$,

$$
\begin{gathered}
\frac{a F}{2 r_{1}}\left(a^{2}-A^{2}-13 b^{2}\right)+\frac{3 a r_{1} E}{2}, \\
2 W=\left(b^{2}-\frac{1}{2} A^{2}\right) I-2 a b[2 \pi-\Omega(P Z)]+\frac{3}{2} b V\left(A^{2}+b^{2}\right) \Omega_{1}(P Z) \\
+\frac{a F}{r_{1}}\left(a^{2}-A^{2}-13 b^{2}\right)+3 a r_{1} E .
\end{gathered}
$$

The potential $W$ may also be considered due to the circular plate $A B$, supposing the density at any interior radius $O Q^{\prime}=r$ is $a-r$; and then, expressed as a double integral

$$
W=\iint \frac{(a-r) r d r d \theta}{P Q^{\prime}},
$$

(9) $\frac{d W}{d a}=\iint \frac{r d r d \theta}{P Q^{\prime}}=$ potential of the end plate of uniform density

$$
\begin{gathered}
=\int\left(a^{2}+a A \cos \theta\right) \frac{d \theta}{P Q}-b[2 \pi-\Omega(P Z)], \\
\frac{d W}{d A}=\iint \frac{-(a-r)(r \cos \theta+A) r d r d \theta}{P Q^{\prime 3}},
\end{gathered}
$$




$$
\frac{d W}{d b}=\iint \frac{-(a-r) r d r \cdot b d \theta}{P Q^{\prime 3}}
$$

and

$$
a \frac{d W}{d a}+A \frac{d W}{d A}+b \frac{d W}{d b}=2 W,
$$

the homogeneity condition of $W$, of two dimensions in length.

65. Other double integrals required in the calculation are

$$
2 \pi-\Omega(P Z)=\iint \frac{r d r \cdot b d \theta}{P Q^{3}},
$$

the apparent area or normal attraction of the $\operatorname{disc} A B$;

$$
\begin{aligned}
\Omega_{1}(P Z) & =\iint \frac{V\left(A^{2}+b^{2}\right) d r \cdot b d \theta}{P Q^{3}}, \\
I & =\iint \frac{d r d \theta}{P Q^{\prime}},
\end{aligned}
$$

the intractable integral (3) $\S 64$ which is the potential of the disc $A B$, with density $r^{-1}$;

$$
\begin{aligned}
& \iint \frac{r \cos \theta d r d \theta}{P Q^{\prime 3}}=\iint \frac{(r+A \cos \theta) \cos \theta-A \cos ^{2} \theta}{P Q^{\prime 3}} d r d \theta \\
& \quad=\int\left(\frac{1}{P O}-\frac{1}{P Q}\right) \cos \theta d \theta-\int \frac{A \cos ^{2} \theta}{P Z^{2}}\left(\frac{a+A \cos \theta}{P Q}-\frac{A \cos \theta}{P O}\right) d \theta \\
& \quad=\frac{a}{A} \int \frac{d \theta}{P Q}-\frac{V\left(A^{2}+b^{2}\right)}{A b} \Omega_{1}(P Z)+v\left(A^{2}+b^{2}\right) \int \frac{\cos \theta d \theta}{P Z^{2}}
\end{aligned}
$$

from $(17) \S 47$, and the third integral vanishes ;

$$
\iint \frac{r^{2} d r d \theta}{P Q^{\prime 3}}=I-2 a \int \frac{d \theta}{P Q}+\frac{V\left(A^{2}+b^{2}\right)}{b} \Omega_{1}(P Z),
$$

(6) $\frac{1}{b} \frac{d W}{d b}=I-2 a \int \frac{d \theta}{P Q}+\frac{V\left(A^{2}+b^{2}\right)}{b} \Omega_{1}(P Z)-\frac{a}{b}[2 \pi-\Omega(P Z)]$,

$$
\begin{aligned}
A \frac{d}{d} \frac{W}{A} & =2 W-a \frac{d}{d} \frac{W}{u}-b \frac{d W}{d b} \\
& =-\frac{1}{2} A^{2} I+\frac{1}{2} b V\left(A^{2}+b^{2}\right) \Omega_{1}(P Z)-\frac{a F}{r_{1}}\left(a^{2}-A^{2}+3 b^{2}\right)+a r_{1} E,
\end{aligned}
$$

$$
\frac{d P}{d b}=-2 \pi A \frac{d W}{d A}, \quad \frac{d P}{d A}=2 \pi A \frac{d W}{d b} .
$$


66. The Stokes function $P$ can be found by an integration of Viriamu Jones's $M$, but an infinite constant comes in when the coil stretches from $b$ to $\infty$; so returning to a coil extending from 0 to $b$, or from $A B$ to the parallel plane through $P$,

$$
\frac{d^{2} P}{d a d \theta}=\frac{d p M}{d \theta}=\frac{a^{2} A^{2} \sin ^{2} \theta}{M Q^{2}} \frac{b}{P Q}
$$

and since

$$
\begin{gathered}
a^{2}=M Q^{2}-2 A \cos \theta(a+A \cos \theta)+A^{2} \cos 2 \theta \\
\frac{d P}{d \theta}=A^{2} \sin ^{2} \theta \int \frac{b d a}{P Q}-2 A^{2} \sin ^{2} \theta \cos \theta \int \frac{a+A \cos \theta}{M Q^{2}} \frac{b d a}{P Q} \\
+A^{2} \sin ^{2} \theta \cos 2 \theta \int \frac{1}{M Q^{2}} \frac{b d a}{P Q} \\
=A^{2} b(1-\cos 2 \theta) \operatorname{th}^{-1} \frac{a}{P Q+P O}+2 A^{3} \sin ^{2} \theta \cos \theta\left(\operatorname{th}^{-1} \frac{b}{P Q}-\operatorname{th}^{-1} \frac{b}{P O}\right) \\
-A^{3} \sin \theta \cos 2 \theta\left[I_{2}-\tan ^{-1}\left(\frac{P O}{b} \tan \theta\right)\right],
\end{gathered}
$$

where $I_{2}$ is given in $(7) \S 47$, and the integration of this term by parts with respect to $\theta$ will depend on $\Omega(P Z)$ and $\Omega_{1}(P Z)$; and $P$ is thus composed of $\frac{1}{2} A^{2} b I$ and I., II., and III. E. I.'s, which can be evaluated in the preceding manner.

The intractable integral

(4) $I=\int 2 \operatorname{th}^{-1} \frac{a}{P Q+P O} d \theta=\int \operatorname{th}^{-1} \frac{a+A \cos \theta}{P Q} d \theta-\int \operatorname{th}^{-1} \frac{A \cos \theta}{P O^{-}} d \theta$, of which the second integral vanishes.

Putting $(a+A \cos \theta) / P Q=\sin \phi^{\prime}$, so that $\phi^{\prime}$ is the angle between $P O$ and $P Z$, and drawing the curve on the Mercator chart connecting latitude $\phi^{\prime}$ and longitude $\theta$, the area will represent $I$, as before in $\S 53$ for $I_{1}$.

For a flat coil in the plane $A B$,

$$
P=\iint \frac{-2 \pi A r \cos \theta d r d \theta}{P Q^{\prime}}
$$

(6) $\frac{d P}{d \theta}=-2 \pi A \cos \theta(P Q-P O)+\pi A^{2}(1+\cos 2 \theta) \operatorname{th}^{-1} \frac{a}{P Q+P O}$, so that

$$
P=\frac{1}{2} \pi A^{2} I \text { and I., II., and III. E. I.'s. }
$$




\section{Legendre's discussion of the oblique cone.}

67. The method of discussion by LEGENDRE of the surface of the oblique cone in his Fonctions elliptiques I, p. 329, may be resumed here, because his $\Phi$, the angle of the sector of the developed surface, and $\Omega$ the conical angle of opening of the reciprocal cone are connected by the relation

$$
\Phi+\Omega=2 \pi,
$$

so that one calculation implies the other.
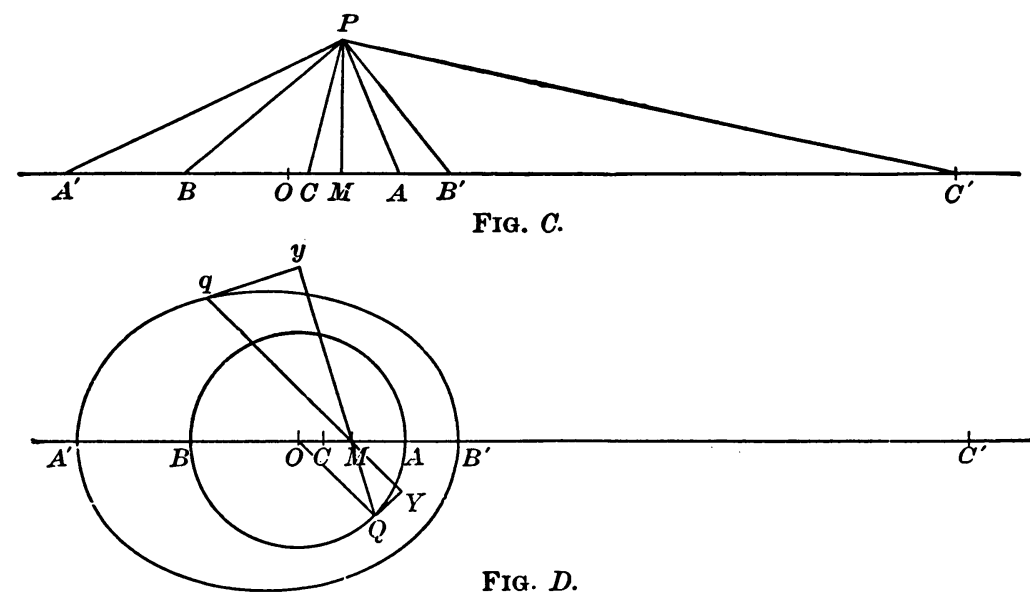

Adopting LEGENDRE's notation for his oblique cone on a circular base, figures $C$ and $D$, and considering it as the reciprocal of the cone discussed previously in figs. $A$ and $B$, then $P M$ the perpendicular from the vertex $P$ on the plane of the circular base $A B$ making an angle $\lambda$ with $P C$ the bisector of the angle $A P B$, and the edges $P A^{\prime}, P B^{\prime}$ of the reciprocal cone making an angle $\lambda^{\prime}$ with $P C$, and putting (LEGENDRE)

(2) $A O=O B=r, \quad O M=f, \quad M P=h, \quad P A=\alpha, \quad P B=\alpha^{\prime}$,

in LEGENDRE's notation.

$$
\begin{gathered}
\sin P A C=\sin \left(\lambda^{\prime}+\lambda\right)=\frac{h}{\alpha}, \quad \cos \left(\lambda^{\prime}+\lambda\right)=\frac{r-f}{\alpha}, \\
\sin P B C=\sin \left(\lambda^{\prime}-\lambda\right)=\frac{h}{\alpha}, \quad \cos \left(\lambda^{\prime}-\lambda\right)=\frac{r+f}{\alpha^{\prime}}, \\
\cos 2 \lambda=\frac{r^{2}-f^{2}+h^{2}}{\alpha \alpha^{\prime}}, \quad \sin 2 \lambda=\frac{2 f h}{\alpha \alpha^{\prime}}, \\
\cos 2 \lambda^{\prime}=\frac{r^{2}-f^{2}-h^{2}}{\alpha \alpha^{\prime}}, \quad \sin 2 \lambda^{\prime}=\frac{2 r h}{\alpha \alpha^{\prime}}, \\
\cos \lambda \sin \lambda^{\prime}=\frac{h\left(\alpha^{\prime}+\alpha\right)}{2 \alpha \alpha^{\prime}}, \quad \sin \lambda \cos \lambda^{\prime}=\frac{h\left(\alpha^{\prime}-\alpha\right)}{2 \alpha \alpha^{\prime}}, \\
\frac{\tan \lambda}{\tan \lambda^{\prime}}=\frac{\alpha^{\prime}-\alpha}{\alpha^{\prime}+\alpha}=\cos \theta,
\end{gathered}
$$


If $P q$ is the generating line of the reciprocal cone perpendicular to the plane $P Q Y$, making an angle $\beta$ with $P M$, and $c$ denotes $\cos A O Q$,

$$
\begin{gathered}
\cot \beta=\frac{M Y}{P M}=\frac{r-f c}{h}=\frac{\sin 2 \lambda^{\prime}-c \operatorname{am} 2 \lambda}{\cos 2 \lambda-\cos \lambda^{\prime}}, \\
\cos \beta \cos 2 \lambda^{\prime}+\sin \beta \sin 2 \lambda^{\prime}=\cos \left(2 \lambda^{\prime}-\beta\right) \\
=\cos \beta \cos 2 \lambda+c \sin \beta \sin 2 \lambda^{\prime}=\cos \beta^{\prime},
\end{gathered}
$$

if $\beta^{\prime}$ denotes the angle between $P Q^{\prime}$ and $P M^{\prime}$ the perpendicular on the other circular section of the cone in fig. $D$; so that

$$
\beta+\beta^{\prime}=2 \lambda^{\prime},
$$

or the sum of the angles which $P Q^{\prime}$ makes with $P M, P M^{\prime}$ is constant, and these are the focal lines of the reciprocal cone in fig. $A$; hence the fundamental property of a sphero-conic.

68. LEGENDRE denotes the angle of the sector $A P Q$ when developed by $\Phi$, and the angle $A O Q$ by $\omega$, so that

and, writing $c$ for $\cos \omega$,

$$
\begin{aligned}
& P Q^{2} d \Phi=P Y \cdot r d \omega, \\
& \Phi=\int \frac{P Y^{2}}{P Q^{2}} \cdot \frac{r d \omega}{P Y},
\end{aligned}
$$

$$
P Q^{2}=r^{2}-2 r f c+f^{2}+h^{2}, \quad P Y^{2}=(r-f c)^{2}+h^{2} .
$$

The angle $Q P Y$ is the $\psi$ of LEgendre, and to reduce $\Phi$ to our form, put

Then, as before in $\S 29$,

$$
\begin{gathered}
m^{2}(\sigma-s)=\frac{P Q^{2}}{P Y^{2}}=\sec ^{2} \psi=\frac{r^{2}-2 r f c+f^{2}+h^{2}}{(r-f c)^{2}+h^{2}}, \\
m^{2}\left(s_{2}-s\right)=\frac{Q Y^{2}}{P Y^{2}}=\tan ^{2} \psi=\frac{f^{2}\left(1-c^{2}\right)}{(r-f c)^{2}+h^{2}}, \\
m^{2}\left(\sigma-s_{2}\right)=1 .
\end{gathered}
$$

$$
\begin{aligned}
& m^{2}\left(s_{1}-s\right)=\frac{\alpha \alpha^{\prime}}{h^{2}} \cos ^{2} \lambda\left(\frac{\left(\alpha^{\prime}+\alpha\right)-\left(\alpha^{\prime}-\alpha\right) c}{2 P Y}\right)^{2}, \\
& m^{2}\left(s-s_{3}\right)=\frac{\alpha \alpha^{\prime}}{h^{2}} \sin ^{2} \lambda\left(\frac{\left(\alpha^{\prime}+\alpha\right) c-\left(\alpha^{\prime}-\alpha\right)}{2 P Y}\right)^{2} .
\end{aligned}
$$

Putting $s=s_{2}, c= \pm 1$,

$$
\begin{gathered}
m^{2}\left(s_{1}-s_{2}\right)=\frac{\alpha \alpha^{\prime}}{h^{2}} \cos ^{2} \lambda, \quad m^{2}\left(s_{2}-s_{3}\right)=\frac{\alpha \alpha^{\prime}}{h^{2}} \sin ^{2} \lambda, \\
m^{2}\left(s_{1}-s_{3}\right)=\frac{\alpha \alpha^{\prime}}{h^{2}}, \quad m^{4}\left(s_{1}-s_{2}\right)\left(s_{2}-s_{3}\right)=\frac{f^{2}}{h^{2}},
\end{gathered}
$$


so that $\lambda$ is the modular angle,

$$
\sin ^{2} \lambda=\frac{s_{2}-s_{3}}{s_{1}-s_{3}}=k^{2},
$$

$$
\begin{gathered}
m^{2}\left(s_{1}-\sigma\right)=\frac{\alpha \alpha^{\prime}}{h^{2}} \cos ^{2} \lambda-1=\frac{\alpha \alpha^{\prime}}{h^{2}} \cos ^{2} \lambda^{\prime}, \\
m^{2}\left(\sigma-s_{3}\right)=\frac{\alpha \alpha^{\prime}}{h^{2}} \sin ^{2} \lambda+1=\frac{\alpha \alpha^{\prime}}{h^{2}} \sin ^{2} \lambda, \\
\frac{1}{2} m^{3} V(-\Sigma)=\frac{r}{h} .
\end{gathered}
$$

Again from (11) $§ 67$,

$$
\begin{aligned}
& \tan \left(\beta-\lambda^{\prime}\right)=\tan \left(\lambda^{\prime}-\beta^{\prime}\right)=\tan \frac{1}{2}\left(\beta-\beta^{\prime}\right) \\
& \quad=\tan \lambda \frac{c \cos \lambda \sin \lambda^{\prime}-\sin \lambda \cos \lambda^{\prime}}{\cos \lambda \sin \lambda^{\prime}-c \sin \lambda \cos \lambda^{\prime}}=\tan \lambda \frac{\left(\alpha^{\prime}+\alpha\right) c-\left(\alpha^{\prime}-\alpha\right)}{\alpha^{\prime}+\alpha-\left(\alpha^{\prime}-\alpha\right) c}=\sqrt{\frac{s-s_{3}}{s_{1}-s^{\prime}},}
\end{aligned}
$$

so that as before in $\S 35$,

$$
\begin{aligned}
& d \beta=\frac{f h d c}{(r-f c)^{2}+h^{2}}=d \tan ^{-1} \sqrt{\frac{s-s_{3}}{s_{1}-s}}=\frac{d s}{\sqrt{\left(4 \cdot s_{1}-s \cdot s-s_{3}\right)}}, \\
& \frac{r d \omega}{P Y}=\frac{-r d c}{P Y V\left(1-c^{2}\right)}=\frac{-f r d c}{P Y^{2} \cdot m V\left(s-s_{2}\right)}=-\frac{r}{h} \frac{d s}{m \sqrt{ } S} . \\
& \Phi=\int_{s_{3}}^{s_{2}} \frac{\sigma-s_{2}}{\sigma-s} \frac{r}{h} \frac{d s}{\sqrt{ } S}=\int \frac{\frac{1}{2} V(-\Sigma)}{\sigma-s} \frac{d s}{V^{\prime} s} .
\end{aligned}
$$

We notice now that in LEGENDRE's notation, with

$$
\cos \theta=\frac{\alpha^{\prime}-\alpha}{\alpha^{\prime}+\alpha}, \quad \text { and } \quad \tan \frac{1}{2} \omega=\tan \frac{1}{2} \theta \tan \frac{1}{2} \phi,
$$

equivalent geometrically to a change from the excentric anomaly $\omega$ to the true anomaly $\phi$ in an orbit of excentricity $\cos \theta$,

$$
\begin{gathered}
\frac{\left(\alpha^{\prime}+\alpha\right) c-\left(\alpha^{\prime}-\alpha\right)}{\alpha^{\prime}+\alpha-\left(\alpha^{\prime}-\alpha\right) c}=\frac{\cos \omega-\cos \theta}{1-\cos \omega \cos \theta}=\cos \phi, \\
\sqrt{\frac{s-s_{3}}{s_{1}-s}}=\tan \lambda \cos \phi, \quad \sqrt{\frac{s-s_{3}}{s_{2}-s_{3}}}=\frac{\cos \phi}{\Delta \phi}
\end{gathered}
$$

and the elliptic functions here are a quarter-period out of phase with the preceding in $\S 26$, and

$$
\phi=\operatorname{am}(1-\grave{h}) K \text {. }
$$

Also

$$
\frac{\mathrm{cn}^{2} f K^{\prime}}{\operatorname{dn}^{2} f \bar{K}^{\prime}}=\frac{\sigma-s_{2}}{\sigma-s_{3}} \frac{s_{1}-s_{3}}{s_{1}-s_{2}}=\frac{h^{2}}{\alpha \alpha^{\prime}} \frac{1}{\sin ^{2} \lambda^{\prime} \cos ^{2} \lambda}=\frac{4 \alpha \alpha^{\prime}}{\left(\alpha^{\prime}+\alpha\right)^{2}}=\sin ^{2} \theta
$$

so that

$$
\theta=\operatorname{am}(1-f) K^{\prime}
$$


Stereographic projection of confocal sphero-conics.

69. Describe the unit sphere with center $P$, cutting the focal lines $P M$, $P M^{\prime}$ in $A, B$ and $P Q^{\prime}$ in $C$, so that in the spherical triangle $A B C$ (fig. $E$ )

$$
A B=c=2 \lambda, \quad B C+C A=a+b=\beta+\beta^{\prime}=2 \lambda^{\prime},
$$

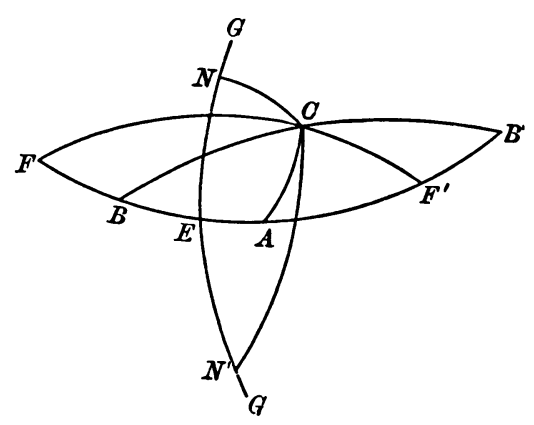

Fig. $E$. and the locus of $C$ is a sphero-conic for constant $a+b$ or $\lambda^{\prime}$ or $f$, the orthogonal confocal sphero-conics having $a-b$ or $h$ constant.

Projecting these confocal sphero-conics stereographically on the tangent plane at $F^{\prime}$, the middle point of $A^{\prime} B$, where the spherical triangle $A^{\prime} B C$ is colunar with $A B C$, and putting $C F=\theta, C F B=\psi$, we can put, according to Burnside, Messenger of Mathematics, vol. 20 (1891), p. 60,

$$
\tan \frac{1}{2} \theta e^{\psi i}=\sqrt{ } c^{\prime} \operatorname{sn}\left(v+u i, c^{\prime}\right) \quad\left[c^{\prime}=\tan 1(\pi-c)\right],
$$

and obtain the orthogonal system of curves given by HoLzM ÜLLER in Isogonale Verwandtschaften.

Here $F A=90+\frac{1}{2} c, F B=90-\frac{1}{2} c$,

$$
\begin{aligned}
\cos a & =\cos \theta \sin \frac{1}{2} c+\sin \theta \cos \frac{1}{2} c \cos \phi \cos b \\
& =-\cos \theta \sin \frac{1}{2} c+\sin \theta \cos \frac{1}{2} c \cos \phi .
\end{aligned}
$$

(4) $2 \cos \theta \sin \frac{1}{2} c=\cos a-\cos b$,

$$
\cos \theta=\frac{\sin \frac{1}{2}(b-a) \sin \frac{1}{2}(b+a)}{\sin \frac{1}{2} a} .
$$

But, writing $w, w^{\prime}$ for $v \pm u i$

$$
\begin{gathered}
\tan \frac{1}{2} \theta=c^{\prime} \operatorname{sn} w \operatorname{sn} w^{\prime}, \quad \cos \theta=\frac{1-c^{\prime} \operatorname{sn} w \operatorname{sn} w}{1+c^{\prime} \operatorname{sn} w \operatorname{sn} w^{\prime}} . \\
\cos \theta=\frac{1-c^{\prime} \operatorname{sn}^{2} v}{1+c^{\prime} \operatorname{sn}^{2} v} \cdot \frac{1+c^{\prime} \operatorname{sn}^{2} u i}{1-c^{\prime} \operatorname{sn}^{2} u i},
\end{gathered}
$$

so that replacing $v$ and $u$ by $f F^{\prime}$ and $h F$, and employing the quadric transformation,

$$
\cos \theta=\frac{\operatorname{dn} f K^{\prime}}{\operatorname{dn} 2 h \bar{K} i},
$$

$$
\begin{gathered}
\sin \frac{1}{2}(b+a)=\operatorname{dn} f K^{\prime}, \quad \cos \frac{1}{2}(b+a)=K^{\prime} \operatorname{sn} f K^{\prime}, \\
\frac{\sin \frac{1}{2}(b-a)}{\sin \frac{1}{2} c}=\frac{1}{\operatorname{dn} 2 K i}=\frac{\operatorname{cn} 2 h K}{\operatorname{dn} 2 h K}=\operatorname{sn}(1-2 h) K, \\
\sin \frac{1}{2}(b-a)=K \operatorname{sn}(1-2 h) K, \\
\cos \frac{1}{2}(b-a)=\operatorname{dn}(1-2 h) K, \\
k=\sin \frac{1}{2} c, \quad k^{\prime}=\cos \frac{1}{2} c .
\end{gathered}
$$


Thus $h=\frac{1}{2}$ makes $a=b$, and the stereographic projection of the great circle $G H$ is a circle, the coalescence of two branches of a quartic curve in HolzMÜLLER's diagram.

From NAPIER's formula

$$
\frac{\cos \frac{1}{2}(A-B)}{\cos \frac{1}{2}(A+B)}=\frac{\tan \frac{1}{2}(a+b)}{\tan \frac{1}{2} c}=\frac{\operatorname{din} f K^{\prime}}{\kappa \operatorname{sn} \frac{K^{\prime}}{\cos }}=\frac{1}{\operatorname{cn}(1-f) K^{\prime}}
$$

we deduce

and from

$$
\tan \frac{1}{2} A \tan \frac{1}{2} B=\frac{1-\operatorname{cn}(1-f) K^{\prime}}{1+\operatorname{cn}(1-f) K^{\prime}} ;
$$

$$
\frac{\sin \frac{1}{2}(A-B)}{\sin \frac{1}{2}(A+B)}=\frac{\tan \frac{1}{2}(a-b)}{\tan \frac{1}{2} c}=\frac{k^{\prime} \operatorname{sn}(2 h-1) K}{\operatorname{dn}(2 h-1) K}=-\operatorname{cn} 2 h K
$$

we deduce

$$
\begin{gathered}
\tan \frac{1}{2} A \div \tan \frac{1}{2} B=\frac{1-\operatorname{cn} 2 h K}{1+\operatorname{cn} 2 h K}, \\
\tan ^{2} \frac{1}{2} A=\frac{1-\operatorname{cn}(1-f)}{1+\operatorname{cn}\left(1-\frac{f}{f)} \cdot \frac{1-\operatorname{cn} 2 h}{1+\operatorname{cn} 2 h},\right.}
\end{gathered}
$$

$$
\text { cn } A=\frac{\operatorname{cn}(1-f)+\operatorname{cn} 2 h}{1+\operatorname{cn}(1+f) \operatorname{cn} 2 h}, \quad \sin A=\frac{\operatorname{sn}(1-f) \operatorname{sn} 2 h}{1+\operatorname{cn}(1-f) \operatorname{cn} 2 h},
$$

$$
\tan ^{2} \frac{1}{2} B=\frac{1-\operatorname{cn}(1-f)}{1+\operatorname{cn}(1-f)} \cdot \frac{1+\operatorname{cn} 2 h}{1-\operatorname{cn} \frac{2 h}{2 h}},
$$

(20) $\cos B=\frac{\operatorname{cn}(1-f)-\operatorname{cn} 2 h}{1-\operatorname{cn}(1-f) \operatorname{cn} \frac{2 h}{2}}, \quad \sin B=\frac{\operatorname{sn}(1-f) \operatorname{sn} 2 h}{1-\operatorname{cn}(1-f) \operatorname{cn} 2 h}$, and so on.

70. If $N, N^{\prime}$ are the poles of the circular sections of the cone, or the foci of the sphero-conics of the reciprocal cone,

$$
\sin E N=\frac{\tan \frac{1}{2} c}{\tan \frac{1}{2}(a+b)}=\cos \theta=\operatorname{cn}(1-f) K^{\prime},
$$

and we find by Spherical Trigonometry and

$$
\cos C N, C N^{\prime}=\cos C E \cos E N \pm \sin C E \sin E N \sin C E A
$$

$$
\cos C E=\frac{\cos \frac{1}{2}(a+b) \cos \frac{1}{2}(a-b)}{\cos \frac{1}{2} c}=\operatorname{sn} f \operatorname{dn}(1-2 h),
$$

$$
\cos E N=\frac{\sqrt{ } \sin s \sin (s-c)}{\cos \frac{1}{2} c \sin \frac{1}{2}(a+b)}=\operatorname{sn}(1-f),
$$

(5) $\sin C E \sin C E A=\frac{V\{\sin s \sin (s-a) \sin (s-b) \sin (s-c)\}}{\sin \frac{1}{2} c \cos \frac{1}{2} c}=\operatorname{cn} f \operatorname{cn}(1-2 h)$, so that

(6) $\cos C N, C N^{\prime}=\frac{\operatorname{sn} f \operatorname{cn} f}{\operatorname{dn} \frac{f}{f}}[\operatorname{dn}(1-2 h) \pm k \operatorname{cn}(1-2 h)]=\operatorname{sn} 2 f F^{\prime} \operatorname{dn}\left(\frac{1}{2} \pm h\right) F$ ， 
and

$$
\cos C N \cos C N^{\prime}=c^{\prime} \mathrm{sn}^{2} 2 f F^{\prime \prime}, \text { a constant, }
$$

a fundamental property of the sphero-conic.

71. If $d A$ denotes the element of plane area cut out by consecutive quartic curves,

$$
d A=c^{\prime} \text { en } w \operatorname{dn} w \text { en } w^{\prime} \operatorname{dn} w^{\prime} \cdot F \cdot d h \cdot F^{\prime} \cdot d f
$$

and if $d w$ denotes the corresponding conical angle,

$$
d w=4 \cos \Psi \frac{1}{2} \theta d A=4 \frac{c^{\prime} \operatorname{cn} w \operatorname{dn} w \operatorname{cn} w^{\prime} \mathrm{dn} w^{\prime}}{\left(1+c^{\prime} \operatorname{sn} w \operatorname{sn} w^{\prime}\right)^{2}} F F^{\prime} d f d h,
$$

and putting $w, w^{\prime}=\frac{1}{2} f K^{\prime} \pm h K i$, this becomes by the quadric transformation,

$$
\begin{aligned}
& d K=4 \frac{1-k \frac{\left(\mathrm{dn}^{4} w-k^{2}\right)\left(\mathrm{dn}^{4} w^{\prime}-k^{2}\right)}{\left(1+k-k^{2}\right)^{2} \mathrm{dn}^{2} w \mathrm{dn}^{2} w^{\prime}}}{\left(1+\frac{k^{\prime 2} \frac{1}{\operatorname{sn} w \operatorname{cn} w \operatorname{sn} w^{\prime} \operatorname{cn} w^{\prime}}}{\operatorname{dn} w \operatorname{dn} w^{\prime}}\right)^{2}} \frac{1}{2}(1+k)^{2} K K^{\prime} d f d h \\
& =\frac{2}{1-k^{2}} \frac{\left(\operatorname{dn}^{4} w-k^{2}\right)\left(\mathrm{dn}^{4} w^{\prime}-k^{2}\right)}{\left(\operatorname{dn} w \operatorname{dn} w^{\prime}+k^{\prime 2} \operatorname{sn} w \operatorname{cn} w \operatorname{sn} w^{\prime} \operatorname{cn} w^{\prime}\right)^{2}} K K^{\prime} d f d h \\
& =2 \frac{\operatorname{dn}^{2}\left(w+w^{\prime}\right) \mathrm{dn}^{2}\left(w-w^{\prime}\right)-k^{2}}{\operatorname{dn}^{2}\left(w-w^{\prime}\right)} K K^{\prime} d f d h \\
& =2\left(\mathrm{dn}^{2} f K^{\prime}-\frac{k^{2}}{\mathrm{dn}^{2} 2 h K_{i}}\right) K K^{\prime} d f d h,
\end{aligned}
$$

in which the variables are separated.

Performing the integrations between limits such that $h$ ranges from 0 to 1 , and $f$ from $f$ to 1 and doubling for the whole conical angle $\Omega$,

$$
\begin{aligned}
& \iint \mathrm{dn}^{2} f K^{\prime} K K^{\prime} d f d h=K\left[(1-f) H^{\prime}-\mathrm{zn} f K^{\prime}\right] \\
& \iint \frac{k^{2}}{\operatorname{dn}^{2} 2 h K i} K K^{\prime} d f d h=\iint\left[1-\mathrm{dn}^{2}(1-2 h) K\right] K K^{\prime} d f d h \\
&=(1-f) K^{\prime} \int_{0}^{1}\left[1-\mathrm{dn}^{2}(1-2 h) K\right] K d h \\
&=(1-f) K^{\prime}(K-H),
\end{aligned}
$$

so that$$
\text { (13) } \Omega=4(1-f)\left(K H^{\prime}+K^{\prime} H-K K^{\prime}\right)-4 K \mathrm{z} f K^{\prime}=2 \pi(1-f)-4 K \mathrm{zn} f K^{\prime} \text {, }
$$

as before, for the complete cone; but (10) shows that the apparent area of any curvilinear quadrilateral, bounded by two pairs of HolzmüLLER's curves can be expressed in the same manner by the I. and II. E. I.

Looking back over the calculation we conclude that the Stokes function has the advantage in simplicity over the potential function, except for the case of the circular ring of $\S 44$.

1 StAPLe InN, London, W. C., January 14, 1907. 